\title{
Abstracts of the 42nd Annual Scientific Meeting of the Nutrition Society of Australia, QT Hotel, Canberra, Australia, 27-30 November 2018
}

\author{
Sandra Iuliano ${ }^{1, *}$, Tanya Lawlis ${ }^{2}$ and Alison Coates ${ }^{3(0)}$ \\ 1 Department of Endocrinology, University of Melbourne/Austin Health, West Heidelberg 3081, Australia \\ 2 School of Rehabilitation and Exercise Sciences, University of Canberra, Bruce 2617, Australia; \\ Tanya.Lawlis@canberra.edu.au \\ 3 School of Health Sciences, University of South Australia, Adelaide 5001, Australia; \\ alison.coates@unisa.edu.au \\ * Correspondence: sandraib@unimelb.edu.au; Tel.: +61-438-215-615
}

Received: 31 January 2019; Accepted: 6 February 2019; Published: 6 March 2019

check for updates

\begin{abstract}
The 42nd Annual Scientific Meeting of the Nutrition Society of Australia was held in Canberra, Australia from November 27 to 30, 2018. The theme of the meeting was Nutrition Science: The nexus between health policy and practice. Abstracts were submitted from 21 countries. Two-hundred-and-forty-four registrants attended the conference and 175 papers were presented consisting of 14 plenary, 77 oral and 84 poster presentations. This issue presents the proceedings of this meeting in the form of abstracts for papers presented at the conference.
\end{abstract}

Keywords: dietary patterns; food sustainability; macronutrients; micronutrients; nutrition policy

\section{Plenary Presentations}

\subsection{Factors Influencing Dietary Habits during Emerging Adulthood}

M. Allman-Farinelli

Charles Perkin Centre, University of Sydney, NSW, Australia

As adolescents emerge into adulthood they undergo physical, emotional, social and intellectual changes that impact on their dietary intakes. Emerging adulthood is generally regarded as 18 to 25 years old. During this phase, young adults develop further independence and sense of identity as they leave school, begin tertiary education or a career and may move out of the parental home. Among adults the youngest have the poorest quality diets. Emerging adults have energy-dense diets that are characterised by low fruit and vegetable intakes and higher intakes of discretionary foods and beverages rich in sodium and added sugars. Thus, it is not surprising to find it is these young males who gain the most BMI units each year and young females gain most BMI units between 18 and 29 years $\left(0.2\right.$ and $\left.0.15 \mathrm{~kg} / \mathrm{M}^{2}\right)$. Young adults in the lower half versus those in the higher half of the population for socioeconomic advantage have diets of a higher energy-density. Lower educational attainment is generally indicative of poorer quality diet. Emerging adults may be more susceptible to the obesogenic food environment and as they eat more food prepared outside the home and are price-sensitive, they are driven to cheap fast foods. 


\subsection{Feeding Our Microbes for Gut Health and Beyond}

D. Belobrajdic

CSIRO Health and Biosecurity, Adelaide, SA, Australia

There is growing recognition of the role of diet and other environmental factors in modulating the composition and metabolic activity of the human gut microbiota and subsequent impact on the development of noncommunicable diseases such as diabetes, cardiovascular diseases and certain cancers. In particular, fibre is a key dietary constituent that promotes gut health, as microbes primarily located in the large bowel can ferment carbohydrates and produce short-chain fatty acids which contribute to the integrity and proper functioning of the large bowel, as well as regulate various metabolic processes systemically. However, these affects are largely dependent on the type of dietary fibre consumed. Although we continue to better understand how specific dietary fibres can influence gut and metabolic health, many populations in developed countries struggle to achieve dietary fibre intakes at recommended levels for proper functioning of the gut. To address this, our research has focused on developing new varieties of staple wholegrain cereals such as wheat, barley and rice that contain enhanced levels of specific dietary fibres to help populations increase their fibre intake to levels that promote gut and metabolic health.

\subsection{The Future and Food Security}

W. Bryden

University of Queensland, QLD, Australia

The massive increase in the human population is precipitating a cascade of environmental, economic, political and cultural changes that have far-reaching implications for the provision of an adequate global food supply. In the future, increased agricultural productivity must come from a reduced land area and resource base. Another "Green Revolution" is required but today's revolution must be different. It is no longer possible or responsible to use unlimited water and chemical inputs to increase production. To achieve new food producing systems that are science-based with low resource input, agricultural R\&D must increase substantially. Education will be equally important, as consumer attitudes will determine the eventual acceptance of new technologies and adoption of different patterns of food consumption. We will need to reinvent our diets to meet our nutritional requirements for optimal health and in so doing consume fewer calories and less meat. Moreover, to maintain a viable food supply, farmers must be paid realistic prices and waste reduced throughout the food supply chain. Part of the solution to feeding the planet is to have nutrition, public health, agriculture and the food industry working together to solve interconnected problems. Future wellbeing depends on a sustainable food system that continues to deliver optimal health with minimal impact on the environment. Visionary public policy, both national and international, must be a major instrument if our food systems are to evolve in a sustainable manner.

\subsection{Climate Change, Limits and the Global Food Supply—What Is the Role of Nutrition Science \\ C. Butler}

Flinders University, South Australia, Australia

Elites, from Henry Kissinger in 1974 to the framers of the 2030 Sustainable Development Goals, have made extravagant, unrealised promises to greatly reduce hunger. Today, over 800 million people are estimated to experience macronutrient undernourishment, five famines are acknowledged and billions more are micronutrient deficient. Hundreds of millions of others are obese. Global nutritional inequality mirrors many other forms of inequality. Neoliberal and other ideologies contribute: many people with power benefit from inequality and collectively act to reduce understanding of its deep causes and remedies. In parallel, little progress has been made in reducing the rate of 
anthropogenic climate change. Climate change and other environmental limits are already worsening nutrition, perhaps for billions. Climate change will further lower micronutrient concentrations in food, worsen heat waves, droughts, fires and floods, and inundate fertile coastal land. Humanity's trajectory risks catastrophe, perhaps even a "fortress world", from which Australians will not be immune. Nutrition science cannot change global macropolitics, but its practitioners can better integrate, appreciate and champion the importance of environmental factors. For example, reduced ingestion of animal products and highly processed foods will mitigate climate change, consume less fossil fuel and improve human health. Such strategies provoke opposition; however, the current trajectory is untenable.

\subsection{Dietary Strategies for Healthy Ageing \\ D. Cameron-Smith}

\section{University of Auckland, Auckland, New Zealand}

Increasing life expectancy and declining birth rates after the baby boomer generation is rapidly skewing population demographics, such that the proportion of people over the age of 65 years is dramatically increasing. Ageing brings with it many complex health challenges, with nutrition exerting a profound impact on the risk and severity of many forms of ill-health and disease. Despite significant declines in the functioning of all tissues and organs it remains a widely held myth that somehow digestion remains largely unaffected. This persists despite the well-established increased prevalence of overt gastrointestinal (GI) diseases and micronutrient deficiencies, particularly for those micronutrients that require complex coordinated GI function. Beyond the basic micronutrients, even less is known of the downstream impacts this exerts on the metabolic pathway that are dependent upon micronutrients as substrates or cofactors. Yet another added complexity is the significant changes in total nutrient intake, including altered meal composition and eating patterns, underpinned in part by changing hedonic, appetite and satiety cues. Therefore, the specific nutritional needs of an ageing population remain largely unknown, with insufficient fundamental knowledge of the changes in nutritional needs that occur along the continuum of ageing.

\subsection{Nutritional Strategies to Ameliorate Heat Stress in Livestock}

\section{J. Cottrel}

University of Melbourne, Parkville, VIC, Australia

Heat stress is a common problem during seasonal heat waves, where growth and reproductive performance is compromised. When future drivers such as the action of climate change and increased participation in tropical agriculture are factored in the issue of producing livestock under hotter climates is an issue that is going to continue to increase in prominence. The use of nutrition as a management strategy against heat stress is not a new concept. However advances in the understanding of the biology of heat stress in livestock has provided new opportunities for the development of amelioration strategies. Many livestock species lack an active sweat response to heat, and instead rely on other means such as redistributing blood from the visceral organs to the skin and increased panting. In this heat stressed state deprivation of blood flow to the intestinal tract can compromise barrier function, resulting in bacterial translocation, inflammatory responses and oxidative stress. Furthermore excessive panting can trigger alterations in acid-base balance. Recently supplementation of compounds such as antioxidants, betaine, chromium and phytochemicals have been proved effective or being tested in mitigating some certain impacts of heat stress in pigs and promise to be an important addition to existing management strategies. 


\subsection{The Future of Health Research Funding in Australia}

A. Kelso

CEO, National Health and Medical Research Council, Canberra, ACT, Australia

Australian health and medical research has entered a new era of opportunity with the introduction of the Medical Research Future Fund (MRFF) and the Biomedical Translation Fund, in addition to National Health and Medical Research Council (NHMRC) funding. While NHMRC remains the biggest player, rising disbursements from the MRFF will substantially increase the total budget available for publicly funded health and medical research. NHMRC and the MRFF have different funding models, the first mainly supporting investigator-initiated research underpinned by peer review across all health topics, and the second focused on priority-driven research delivered through a mix of competitive and targeted mechanisms. At the same time, NHMRC is undertaking a major reform of its grant program to address issues that have weighed on the Australian health and medical research sector in recent years. This changing landscape of research funding offers new opportunities for researchers in human nutrition and related fields that underpin individual and community health and wellbeing.

\subsection{The Science and Politics of Nutrition Evidence Generation, Synthesis and Translation}

\section{Lawrence}

Institute for Physical Activity and Nutrition, Deakin University, Burwood, VIC, Australia

Dietary risk factors are leading contributors to the Australian burden of disease. Nutrition science provides theories, principles, procedures and research methods for generating, synthesising and translating evidence to inform nutrition guidelines and policy activities to tackle these dietary risk factors. However, nutrition evidence does not emerge by, or speak for, itself. Its generation, synthesis and translation are influenced by human involvement. Inevitably, politics shapes which research questions are (not) asked and funded and by whom, how procedures for rating the certainty of the resulting body of nutrition evidence are constructed and applied and whose worldviews then prevail in formulating nutrition guidelines and policy activities from among the variety of nutrient-, food-, diet- and food system-oriented options available. Novel approaches are being proposed to help illuminate and manage nutrition science and political interactions. Approaches for tackling bias in evidence generation are gaining traction. An innovative approach drawing on theory and a logic model to strategically inform evidence synthesis and translation for nutrition guidance and policy activities will be described. These approaches aim to strengthen evidence use decision-making processes for more effective and safe nutrition guidelines and policy activities.

\subsection{The Gut Microbiome and Bone Health}

\section{McCabe}

Michigan State University, USA

It is clear that intestinal microbial dysbiosis, barrier breaks and inflammation can promote bone loss. In fact, many conditions of bone loss also have altered gut function (such as menopause, inflammatory bowel disease and type 1 diabetes). The gut-bone signalling axis not only represents a signal for bone loss but can also serve as a novel therapeutic target for preventing osteoporosis. We have identified links between gut and bone health and can manipulate the intestinal environment in a variety of ways, for example with antibiotics to deplete the microbiome and with probiotics to enhance the microbiome. The latter leads to a clear benefit to both the intestine and bone. Our studies have focused on the probiotic lactobacilli reuteri, which has anti-TNF properties. Our data indicate that this probiotic can influence bone indirectly and directly through pathways unique to these bacteria. Effects of the probiotic in different disease models, an overarching model and future directions will be discussed. 


\subsection{The Ecology of Nutrition and Dysbiosis—Drawing a Direct Line between Personal and Planetary Health}

\section{S. Prescott}

University of Western Australia, WA, Australia

Nutrition is arguably the most significant determinant of the development and future health of all organ systems, and by extension, a major factor in each individual reaching their potential through resiliency, quality of life and ultimately, life expectancy. The microbiome revolution has shown us that everything is interconnected - the ecology of our food, our personal biomes, our health, our societies, and the health and biodiversity of the wider environment-drawing a direct line between personal and planetary health. At the personal scale, dietary patterns are key in determining the profile and function of the gut microbiome, and are now known to have a fundamental influence on the immune and metabolic functions that determine health across life. Adverse western dietary changes are amongst the most significant factors in the rapidly mounting global burden of noncommunicable diseases (NCDs), exacerbating inflammation through multiple pathways, including 'dysbiotic' changes in gut microbiomes. At the planetary scale, dietary patterns and food choices are adding to and perpetuating macroscale dysbiosis through significant impacts on the environment, with clear evidence of differential effects on land use, $\mathrm{CO}_{2}$ production, waste, macroscale biodiversity loss, water use and particulate pollution. At a time of increasing environmental crisis on all scales there is an imperative for integrated ecological solutions which provide sustainable dietary guidance for optimal human nutrition in tandem with issues of long-term food security, environmental, social and economic impacts of human wellbeing into the future. We have the resources and technologies to do this, we must now apply the vision and shared purpose required to achieve this. Our future depends on it.

\subsection{Weight Management across the Lifecourse}

\section{Redman}

Pennington Biomedical Research Center, Louisiana State University, Baton Rouge, LA, USA

With the increasing prevalence of obesity worldwide, understanding effective approaches for weight management has become critical focus for basic, clinical and translational research. The technology boom of the modern day while thought to be a contributor in the obesogenic environment is also being used to intervene and improve health and health behaviours. Modern technologies such as smart phones and accompanying Bluetooth enabled devices allow for individualised treatment recommendations to be delivered to individuals remotely, increased self-monitoring/tracking of health-related data, broader and more rapid dissemination of health information/recommendations and increased patient-dietician/physician contact. The use of technology in weight management programs results in improved long-term weight management and, in most cases, increased cost effectiveness. We have been at the forefront of developing and deploying weight management programs that rely on a smart phone. The foundation of our approach are mathematical models that predict a weight loss (or gain trajectory) for a given individual and use of empirical data such as body weight and activity data obtained from wireless scales and activity trackers to deliver feedback and tailor intervention intensiveness. Our efficacy trials for weight loss and healthy management of weight gain in pregnant women hold promise for wide-scale dissemination in large scale healthcare systems.

\subsection{Micronutrients and Depression}

\section{A. Ruusunen}

School of Medicine, Deakin University, Burwood, VIC, Australia

Depression is the leading source of disability and identification of new targets for prevention and management is imperative. Extensive evidence supports associations between diet quality 
and depression. However, the role of micronutrients remains unclear. Low intake of folate and lower blood concentrations of vitamin D are especially associated with increased risk of depression. Deficiencies of micronutrients such as folate and zinc may predispose to depression. This presentation will discuss the evidence base for these micronutrients as well as folic acid, magnesium and multivitamin supplementations. Potential mechanisms (neurotransmitters, inflammation, HPA-axis function, oxidative stress, neuroplasticity and gut microbiota) will also be discussed. Preliminary experimental evidence suggests some benefits of micronutrient supplementations both in population and clinical samples. Especially, methyl-folate and vitamin D supplementations may promote clinical improvements. However, evidence based on double-blind randomised controlled trials remains limited. Micronutrients may have a specific role in the prevention and treatment of depression, but at the population level, the benefits of whole-diet approach exceed the benefits of individual micronutrients. These findings highlight the fact that improving quality of diet is important for mental health and will lead to increased intake of micronutrients. However, micronutrient supplementations may have a role in treatment of specific nutrient deficiencies.

\subsection{The Relationship between the Mediterranean Dietary Pattern and Musculoskeletal Health in Children,} Adolescents and Adults

\section{A. Welch}

\section{Medical School, University of East Anglia, UK}

The Mediterranean Diet is potentially protective for skeletal muscle and bone health and therefore, for sarcopenia, osteoporosis and risk of fractures. The high cost of these conditions in ageing Western populations means prevention is important. The Mediterranean Diet includes eating more fruits and vegetables, olive oil, nuts, legumes and fish; lower amounts of meat and dairy foods; and moderate amounts of alcohol. The benefits of the Mediterranean Diet are conferred through the macronutrients, and micronutrient vitamins and minerals, contributed by the foods within this pattern. Our previous systematic review investigated the relevance of the Mediterranean Diet to musculoskeletal health in people of all ages (Craig J et al., 2017) and found only two studies relating the incidence of hip fracture to the MD, and one for sarcopenia. A further 15 studies in our evidence map did not meet the criteria of our systematic review. Although the MD is considered a consistent type of eating pattern, the nutrient and food composition is very variable because of the range of definitions and types of foods consumed in different regions of the world. My presentation will discuss and relate the Mediterranean Diet to musculoskeletal health and outcomes.

\subsection{Chronic Effects of Dietary Polyphenols on Health Markers}

\section{G. Williamson}

Department of Nutrition, Dietetics and Food, Monash University, VIC, Australia

Diabetes and cardiovascular disease are global problems, but the risks of developing these conditions have the potential to be lowered by diets high in fruits and vegetables. Plant-derived foods contain naturally-occurring components termed polyphenols (flavonoids and phenolic acids), and according to epidemiological and intervention studies, diets rich in polyphenols reduce the risk developing type 2 diabetes and cardiovascular disease. Effects of polyphenols on biomarkers of health are predominantly observed chronically, with regular intake, and include modulation of postprandial metabolism. Experiments on human cells in vitro have indicated mechanisms of action, complemented by studies on healthy and at risk human volunteers using specific biomarkers. It is now clear that the beneficial effects are on specific cellular targets and are not due to a direct chemical "antioxidant" action. The molecular pathways of absorption and metabolism of the most common dietary polyphenols are well understood, involving action by both endogenous enzymes and by the gut microbiota, and their bioavailability is directly linked to bioefficacy and effect on health biomarkers. The presentation will 
discuss how dietary polyphenols modulate biomarkers of type 2 diabetes and cardiovascular disease risk, and how these effects are influenced by bioavailability and the gut microbiota.

\section{Regular Abstracts}

2.1. The Relationship between Colour and Antioxidant and Phytochemical Properties of Some Commercially Available Australian Honeys

M. Hunter ${ }^{1,2}$, N. D'Cunha ${ }^{1,2}$, E.N. Georgousopoulou ${ }^{1,2,3}$, D. Mellor ${ }^{1,2,4}$ and N. Naumovski ${ }^{1,2}$

${ }^{1}$ Nutrition and Dietetics, University of Canberra, Canberra, ACT, Australia

${ }^{2}$ Collaborative Research in Bioactives and Biomarkers, Canberra, ACT, Australia

${ }^{3}$ Nutrition-Dietetics, Harokopio University, Athens, Greece

${ }^{4}$ School of Life Sciences, Coventry University, Coventry, UK

Honey is the supersaturated sugar solution produced from the pollen and nectar of plants, with its composition and appearance largely influenced by surrounding floral sources and its geographic origin. Honey exhibits a range of potential health benefits, which is proposed to be a result of its composition. This study investigated a selection of commercially available Australian honeys and potential associations between colour and the relatively unexplored phytochemical composition, including antioxidant characteristics. Commercially available Australian honey samples $(n=42)$ were categorised by 'front-of-label' descriptions and analysed for colour intensity (Spectrophotometric method; $\mathrm{ABS}_{450}$; mAU); phytochemical content: total phenolic content (TPC; Folin-Ciocalteu; Gallic Acid Equiv. (GAE)); total flavonoids (TFC; $\mathrm{AlCl}_{3}$; Catechin Equiv. (CE)); and antioxidant characteristics (DPPH, CUPRAC, FRAP; Trolox Equiv. (TE)). The 'Manuka-honey' category was higher in colour intensity (darker) than any other category (all $p<0.05$ ); except for the 'organic-honey' category $(p=0.171)$. Colour intensity was positively correlated with each phytochemical-TPC $(p<0.001)$ and TFC $(p<0.001)$ - and individual antioxidant characteristics (all $p<0.05)$. The findings of this study indicate that more colour intensive (darker) honeys contained higher concentrations of phytochemicals. Conclusively, colour is a potential proxy measure for antioxidant and phytochemical quantification in commercially available Australian honeys.

\subsection{Strawberries-An Underestimated Dietary Source of Folate}

M. Netzel ${ }^{1}$, N. Weber ${ }^{1,2}$, C. Dumler ${ }^{1,2}$, L. Striegel ${ }^{2}$, A. Phan ${ }^{1}$, H. Trieu ${ }^{1}$, M. Rychlik ${ }^{1,2}$ and T. $\mathrm{O}^{\prime}$ Hare $^{1}$

${ }^{1}$ University of Queensland, Coopers Plains, QLD, Australia

2 Technical University Munich, Freising, Germany

Folate is considered as a critical vitamin in many countries and folate deficiency is associated with neural tube defects in newborns. Strawberries are considered a tasty and healthy fruit by many consumers and may potentially be an important dietary source of natural folates, depending on variety and growing environment. Therefore, a broad range of different strawberry varieties and breeding lines was screened for their folate content and profile. Thirty-five Australian-grown strawberry varieties and experimental breeding lines were analysed for folate by stable isotope dilution assay. Total folate content ranged from 70 to $164 \mu \mathrm{g} / 100 \mathrm{~g}$ fresh weight, which was well above the value in the NUTTAB database ( $39 \mu \mathrm{g} / 100 \mathrm{~g}$ fresh weight). Furthermore, folate concentration in the outer strawberry tissue was found to be 1.5-1.7-fold higher compared to the inner tissue of the fruit. 5-Methyltetrahydrofolate, the biologically active form, was the principal vitamer. With these high folate concentrations, a single serve (144 g) of Australian-grown strawberries would deliver up to $59 \%$ of the FSANZ recommended dietary intake (RDI) for folate. Furthermore, the differences between outer and inner tissue could indicate that flatter, longer strawberries may have greater potential to accumulate folate than fruit with a more spherical shape. 
2.3. Biofortified Sweet Corn-A Promising Dietary Source of Zeaxanthin for Age-Related Macular Degeneration

M.E. Netzel ${ }^{1}$, T. Zeilmann ${ }^{1,2}$, K.J. Fanning ${ }^{3}$, M. Rychlik ${ }^{1,2}$ and T.J. O'Hare ${ }^{1}$

${ }^{1}$ University of Queensland, Coopers Plains, QLD, Australia

2 Technical University Munich, Freising, Germany

${ }^{3}$ Department of Agriculture and Fisheries, QLD Government, Coopers Plains, QLD, Australia

Sweet corn is a natural source of zeaxanthin, one of two dietary carotenoids accumulated in the macula and associated with slowing the development of age-related macular degeneration. The aim of the present study was to compare the concentration and bioaccessibility of zeaxanthin in a biofortified sweet corn (HZ23-1) and a standard commercial sweet corn (Garrison). Zeaxanthin content and bioaccessibility was determined in microwave-cooked sweet corn kernels of HZ23-1 and Garrison by HPLC and a static in vitro digestion model, respectively. HZ23-1 had five times higher zeaxanthin-concentration than Garrison (11.8 vs. $2.4 \mathrm{mg} / \mathrm{kg} \mathrm{FW}$ ), but was less bioaccessible ( $29 \%$ vs. $63 \%$ ). Despite an apparently lower bioaccessibility, the absolute release of zeaxanthin was significantly $(p<0.05)$ higher than that released by Garrison (3.4 vs. $1.5 \mathrm{mg} / \mathrm{kg}$ FW). Although biofortified HZ23-1 sweet corn provided 2.3-fold higher matrix-released zeaxanthin than standard Garrison sweet corn, the reduction in relative bioaccessibility was unexpected. It is possible that mechanisms within the kernel endosperm enabling greater zeaxanthin accumulation may also interfere with zeaxanthin release from the endosperm matrix. The mechanism of zeaxanthin storage in the endosperm should be further investigated to further improve the bioaccessibility of zeaxanthin from biofortified sweet corn.

\subsection{Differential Effects of Medium-and Long-Chain Saturated Fatty Acids on Blood Lipid Profile: A Systematic} Review and Meta-Analysis

N. Panth ${ }^{1}$, K. Abbott ${ }^{1}$, C. Dias ${ }^{1}$, KJ. Wynne ${ }^{2}$ and M. Garg ${ }^{1}$

${ }^{1}$ Nutraceutical Research Program, University of Newcastle, Newcastle, NSW, Australia

${ }^{2}$ Department of Diabetes \& Endocrinology, University of Newcastle, Newcastle, NSW, Australia

Saturated fatty acids of different chain lengths may have variable effects on blood lipids and lipoproteins, likely due to their differing routes of digestion, absorption and metabolism. This review investigated whether ingestion of medium- (MCFA) and long (LCSFA)-chain saturated fatty acids have differential impact on blood lipids. Five databases were searched (EMBASE, MEDLINE, CINAHL, Cochrane and Scopus) and clinical trials investigating the effect of MCFA and LCSFA on blood lipid profile were included. Studies were pooled for meta-analysis. The principle summary measure was the mean difference between groups calculated using the random effects model. Eleven cross-over studies and one parallel study were identified with a total of $n=299$ participants (weighted means $\hat{\mathrm{A}} \pm \mathrm{SDs}$ age: $38 \hat{\mathrm{A}} \pm 3 \mathrm{y}$; body mass index (BMI): $24 \hat{\mathrm{A}} \pm 2 \mathrm{~kg} / \mathrm{m}^{2}$ ). Â MCFA were associated with significantly higher high-density lipoprotein cholesterol (HDL-C) (mean difference: $0.11 \mathrm{mmol} / \mathrm{L} ; 95 \% \mathrm{CI}$ : 0.07, 0.15 ) and apolipoprotein A-I (Apo A-I) level (mean difference: 0.08 g/L; 95\% CI: 0.02, 0.14) compared to LCSFA, with no change in total cholesterol. The findings demonstrate a differential effect of MCFA and LCSFA on HDL-C levels. Therefore, dietary recommendations to reduce saturated fatty acids for reducing CVD risk should be limited to foods rich in LCSFA.

2.5. Relationship of Dietary Nitrate Intake from Vegetables with Cardiovascular Disease Mortality: A Prospective Study in a Cohort of Older AUSTRALIANS

A.H. Liu ${ }^{1}$, C.P. Bondonno ${ }^{2}$, J. Russell ${ }^{3}$, V.M. Flood ${ }^{4,5}$, J.R. Lewis ${ }^{2}$, K.D. Croft ${ }^{1}$, R.J. Woodman ${ }^{6}$, W.H. Lim ${ }^{1,7}$, A. Kifley ${ }^{4}$, G. Wong ${ }^{4,8}$, P. Mitchell ${ }^{4}$, J.M. Hodgson ${ }^{2}$ and L.C. Blekkenhorst ${ }^{2}$

${ }^{1}$ The University of Western Australia, Perth, WA, Australia

2 Edith Cowan University, Perth, WA, Australia

${ }^{3}$ The University of Wollongong, Wollongong, NSW, Australia 
4 The University of Sydney, Sydney, NSW, Australia

${ }^{5}$ Westmead Hospital, Westmead, NSW, Australia

${ }^{6}$ Flinders University, Adelaide, SA, Australia

${ }^{7}$ Sir Charles Gairdner Hospital, Nedlands, WA, Australia

${ }^{8}$ Children's Hospital at Westmead, Westmead, NSW, Australia

Short-term trials indicate that inorganic nitrate and nitrate-rich vegetables have vascular health benefits. However, few studies have explored the relationship between nitrate intake and long-term cardiovascular disease (CVD) outcomes. We aimed to investigate the association of vegetable nitrate intake with CVD mortality in a cohort of older Australians. Participants without diabetes or major CVD at baseline (1992-1994) were included from the Blue Mountains Eye Study, a population-based cohort study of men and women aged $\geq 49$ years. Dietary intake was assessed at baseline, 5 years and 10 years of follow-up. Vegetable nitrate intake was estimated using a comprehensive vegetable nitrate database. Cox proportional hazards regression was used to explore the association between vegetable nitrate intake and CVD mortality. During 14 years of follow-up, 188/2229 (8.4\%) participants died from CVD. In multivariable-adjusted analysis, participants in quartile 2 (69.5-99.6 mg/d; HR: 0.53 (95\% CI: $0.35,0.82)$ ], quartile 3 [99.7-137.8 mg/d; HR: 0.51 (95\% CI: 0.32, 0.80)), and quartile 4 (>137.8 mg/d; HR: 0.63 (95\% CI: 0.41, 0.95)) of vegetable nitrate intake had lower hazards for CVD mortality compared to participants in quartile 1 ( $<69.5 \mathrm{mg} / \mathrm{d})$. In older Australian men and women, vegetable nitrate intake was inversely associated with CVD mortality, independent of lifestyle and cardiovascular risk factors.

\subsection{Dietary Flavonoid and Flavonoid Subclass Intake in Australian Adolescents in The Raine Study}

K. Kent ${ }^{1}$, K. Charlton ${ }^{2,3}$ and W. Oddy ${ }^{4}$

${ }^{1}$ University of Tasmania, Launceston, TAS, Australia

${ }^{2}$ School of Medicine, University of Wollongong, Wollongong, NSW, Australia

${ }^{3}$ Illawarra Health and Medical Research Institute, Wollongong, NSW, Australia

${ }^{4}$ Menzies Institute for Medical Research, University of Tasmania, Hobart, TAS, Australia

Dietary flavonoids have been linked to a protection against cardiovascular disease. Flavonoid intake during adolescence has been associated with lower serum lipids in adulthood. However, further investigation of flavonoid intake in Australian adolescents is needed. This study aimed to determine flavonoid and flavonoid subclass intake, and their major dietary sources in Australian adolescents. Adolescents (aged 14 years; $n=1632 ; 51 \%$ male) completed a food frequency questionnaire as part of the Western Australian Pregnancy Cohort (Raine) Study. Food items $(n=212)$ were assigned a content for six flavonoid subclasses using the Phenol-Explorer database, which were summed for total flavonoid intake. Mean daily intake and sources of flavonoids and flavonoid subclasses were determined, and gender differences were investigated. Total flavonoid intake (mean \pm standard deviation) was $208.4 \pm 134.5 \mathrm{mg} /$ day, with a significant difference evident between genders (one-way ANOVA: $\mathrm{F}(1,1630)=1.7, p=0.038$; males: $201 \pm 129 \mathrm{mg} /$ day; females: $215 \pm 139 \mathrm{mg}$ /day). Intake of subclasses ranged from isoflavones $(0.5 \pm 1.4 \mathrm{mg} /$ day), to flavones $(4.5 \pm 4.8 \mathrm{mg} /$ day $)$, anthocyanins ( $21.5 \pm 25 \mathrm{mg} /$ day), flavonols ( $22.5 \pm 16.35 \mathrm{mg} /$ day), flavan-3-ols $(57.6 \pm 51.1 \mathrm{mg} /$ day) and flavanones $(101.6 \pm 97.7 \mathrm{mg} /$ day). Fruit juice $(42.7 \%)$ and tea $(8.5 \%)$ were the major sources of flavonoids. Total fruits and vegetables contributed $30 \%$ and $7 \%$, respectively. This study provides the first focused assessment of flavonoid intake in Australian adolescents, providing a basis to assess the relationship between flavonoid intake and cardiovascular risk factors.

2.7. Almond-Enriched Diet Associated with Reductions in Triglycerides and Systolic Blood Pressure in Middle-Aged to Older Adults

A.M. Coates ${ }^{1}$, S. Morgillo ${ }^{1}$, C. Yandell ${ }^{1}$, A. Scholey ${ }^{2}$, J. Buckley ${ }^{1}$ and A. Hill ${ }^{3}$ 
${ }^{1}$ School of Health Sciences, University of South Australia, Adelaide, SA, Australia

${ }^{2}$ Centre for Human Psychopharmacology, Swinburne University, Melbourne, VIC, Australia

${ }^{3}$ School of Pharmacy and Medical Sciences, University of South Australia, Adelaide, SA, Australia

Nut consumption has been associated with reduce risk of coronary heart disease. This study examined the effect of supplementing habitual diets for 12 weeks with either almonds or carbohydrate rich snack foods on biomarkers of cardiovascular and metabolic health. Participants $(n=151$; overweight/obese, 50-80 years) were randomised to replace $15 \%$ energy intake with either almonds (Almond) or isocaloric snack foods (Control). Body weight, blood lipids, glucose, insulin, blood pressure (BP) and arterial stiffness were measured at baseline and 12 weeks. One hundred and twenty eight participants (78F:50M, $n=63$ almond, $n=65$ control) completed the intervention $\left(\mathrm{M} \pm\right.$ SD: age $64 \pm 8$ years, BMI $30.3 \pm 3.6 \mathrm{~kg} / \mathrm{m}^{2}$, stable medication use: $32 \%$ BP and $19 \%$ lipid lowering). Almond intake significantly lowered triglycerides $(\mathrm{M} \pm \mathrm{SEM}$, almond: $1.30 \pm 0.062 \mathrm{mmol} / \mathrm{L}$ to $1.16 \pm 0.062 \mathrm{mmol} / \mathrm{L}$ vs. control: $1.13 \pm 0.061 \mathrm{mmol} / \mathrm{L}$ to $1.17 \pm 0.061 \mathrm{mmol} / \mathrm{L}, p=0.005)$. A trend toward a greater reduction in systolic BP in the almond group (almond: $132.6 \pm 1.8 \mathrm{mmHg}$ to $128.5 \pm$ vs. control: $132.4 \pm 1.8 \mathrm{mmHg}$ to $131.8 \pm 1.8 \mathrm{mmHg}, p=0.054)$ became significant following removal of participants taking BP medication $(p=0.035)$. There were no significant changes for other cardiometabolic biomarkers. The inclusion of almonds in the diet has the potential to improve aspects of cardiometabolic health.

\subsection{Oat Beta Glucan Enhances the Lipid-Lowering Effects of Phytosterols in Individuals} with Hypercholesterolaemia

J.J.A. Ferguson ${ }^{1}$, E. Stojanovski ${ }^{2}$, L. MacDonald-Wicks ${ }^{3}$ and M.L. Garg ${ }^{1}$

${ }^{1}$ School of Biomedical Sciences and Pharmacy, Nutraceuticals Research Program, University of Newcastle, Callaghan, NSW, Australia

2 School of Mathematics and Physical Sciences, University of Newcastle, Callaghan, NSW, Australia

${ }^{3}$ School of Health Sciences, University of Newcastle, Callaghan, NSW, Australia

Dietary phytosterols (PS) and oat beta glucan (OBG) are known to reduce circulating blood cholesterol levels. The aim of this study was to investigate the effects of dietary supplementation with a novel food (sweet biscuits) enriched with PS ( $2 \mathrm{~g} /$ day) and/or OBG ( $3 \mathrm{~g} /$ day) for six weeks on blood lipids. A $2 \times 2$ factorial, double-blinded, randomised controlled intervention trial was conducted in adults with hypercholesterolaemia. Primary outcomes included fasting total cholesterol (TC), LDL-cholesterol, HDL cholesterol, triglycerides and TC-to-HDL-C ratio (TC:HDL-C). Significant reductions in TC in PS $(p<0.05)$, OBG $(p<0.05)$ and PS-OBG $(p<0.0001)$, LDL-cholesterol in PS $(p<0.05)$, OBG $(p<0.01)$ and PS-OBG $(p<0.001)$, TC:HDL-C in OBG $(p<0.05)$ and PS-OBG $(p<0.01)$ were observed postintervention. Triglycerides significantly reduced in the PS-OBG group only $(p<0.05)$. There was also a significant difference in the mean change in TC and LDL-cholesterol across groups $(p<0.05)$. Plasma HDL cholesterol remained unchanged across all groups. These findings support complementarity between PS and OBG for cholesterol-lowering, and the combination of the two in a single food appears to promote reduction in triglycerides. Implications of these findings support the development of functional foods containing PS and high molecular weight OBG for enhancing lipid-lowering and improving heart health benefits.

\subsection{Association between Changes in Dietary Intake and Lipid-Lowering Therapy: A Prospective Analysis of} Data from The Australian Diabetes, Obesity and Lifestyle Study (AusDiab)

A.M. Gadowski ${ }^{1}$, N. Nanayakkara ${ }^{1}$, D. Magliano ${ }^{2}$, J. Shaw ${ }^{2}$, A. Curtis ${ }^{1}$, S. Zoungas ${ }^{1}$, S. Heritier ${ }^{1}$ and A. Owen ${ }^{1}$

${ }^{1}$ Monash University, Melbourne, VIC, Australia

2 Baker Heart and Diabetes Institute, Melbourne, VIC, Australia 
Lipid-lowering therapy (LLT) for cardiovascular risk reduction should be accompanied by dietary/lifestyle advice. This study examines associations between dietary changes of major food groups (as per Australian Dietary Guidelines) and use of LLT. Data were analysed for 6037 adult participants in the Australian Diabetes, Obesity and Lifestyle Study. Change in food group (vegetable, fruit, cereal, protein and dairy) intake was analysed from baseline (1999-2000) to follow-up (2004-2005). Association between changes in food group intake and use of LLT at baseline was examined using quantile regression, adjusted for known risk factors. Mean age $( \pm S D)$ was $51 \pm 14$ years $(55 \%$ female). At baseline, $904(8 \%)$ were using LLT. Among nonusers, 46\%, 54\%, 6\%, 75\% and $8 \%$ adhered to guidelines for vegetable, fruit, cereal, protein and dairy, respectively, compared to $51 \%, 59 \%, 11 \%$, $77 \%$ and $8 \%$, respectively, for LLT users. In adjusted analyses, median change in dairy intake was lower among users compared with nonusers $(p<0.03)$. There were no differences between users and nonusers for other food groups. Participants on LLT did not differ in recommended intakes compared with nonusers. Given users are at elevated cardiovascular risk, targeted strategies are needed to optimise dietary intake for people requiring LLT to enhance cardiovascular risk reduction.

\subsection{Effect of Adding Milk to Black Tea on Vascular Function in Healthy Men and Women}

J.M. Hodgson ${ }^{1}$, H. Koch ${ }^{2}$, K.D. Croft ${ }^{2}$, A.F. Ahmad ${ }^{3}$ and N.C. Ward ${ }^{3}$

${ }^{1}$ Edith Cowan University, Perth, WA, Australia

${ }^{2}$ University of Western Australia, Perth, WA, Australia

${ }^{3}$ Curtin University, Perth, WA, Australia

There is a lingering concern that the practice of adding milk to tea can blunt the well-established benefits of tea consumption on vascular function. We aimed to investigate the effect of regular consumption of black tea-with and without milk—on vascular function and blood pressure in healthy volunteers. A randomised, controlled, cross-over study was performed in 17 healthy volunteers $(7 \mathrm{men}$ and 10 women; mean age $22.4 \pm 3.0$ years). Participants received each of the following treatments in random order for four weeks, 3 cups / d of (i) hot water, (ii) black tea and (iii) black tea with milk. Black tea increased flow-mediated dilatation (FMD) of the brachial artery compared to the hot water $(1.00 \pm 0.18 \%, p<0.0001)$. Black tea with milk decreased FMD compared to both the hot water $(-0.64 \pm 0.19 \%, p=0.001)$ and black tea $(-1.64 \pm 0.19 \%, p<0.0001)$. Compared with hot water, black tea did not alter blood pressure, while black tea with milk increased systolic blood pressure $(1.1 \pm 0.5 \mathrm{mmHg}, p=0.03)$ and diastolic blood pressure $(2.0 \pm 0.5 \mathrm{mmHg}, p<0.0001)$. Our findings suggest that the addition of milk to black tea alters the short-term impact of regular tea consumption on vascular function and blood pressure in young healthy men and women.

2.11. Is Dietary Nitrate Associated with a Lower Risk of Cardiovascular Disease-Related Complications among Middle-Aged Australian Women

J. Jackson ${ }^{1}$, A. Patterson ${ }^{1}$, L. MacDonald-Wicks ${ }^{1}$, P. Forder ${ }^{1,2}$, L. Blekkenhorst ${ }^{3}$, C. Bondonno ${ }^{3}$, J. Hodgson ${ }^{3}$, N. Ward ${ }^{4}$, J. Byles ${ }^{1,2}$, C. Holder ${ }^{1,2}$, C. Oldmeadow ${ }^{1,2}$ and M. McEvoy ${ }^{1,2}$

${ }^{1}$ University of Newcastle, Newcastle, NSW, Australia

${ }^{2}$ Hunter Medical Research Institute, New Lampton, NSW, Australia

${ }^{3}$ Edith Cowan University Western Australia, Perth, WA, Australia

${ }^{4}$ University of Western Australia, Perth, WA, Australia

Vegetables account for 80 to $90 \%$ of total dietary nitrate intakes. Evidence suggests that dietary nitrate as a nitric oxide donor may help explain the cardiovascular benefits of vegetables. Our aim was to prospectively investigate the association between dietary nitrate intakes and incidence of self-reported cardiovascular disease (CVD) related complications in a population based cohort of middle-aged Australian women (1946-51 cohort of the Australian Longitudinal Study on Women's Health). Women free from disease at baseline and who had complete food frequency questionnaire 
data were included. Generalised estimating equations were used to estimate odd ratios (OR) and $95 \%$ confidence intervals ( $95 \%$ CI). Of the 5324 women included for analysis, 1616 women reported at least one CVD-related complication over 16 years of follow-up. When comparing the highest quartile for total nitrate intake $(>78 \mathrm{mg} / \mathrm{d})$ to the lowest $(<45 \mathrm{mg} / \mathrm{d})$, the fully adjusted OR was $0.94(95 \%$ CI: 0.85 to $1.04 ; p=0.29)$. When comparing the highest quartile for vegetable nitrate $(>65 \mathrm{mg} / \mathrm{d})$ to the lowest $(<35 \mathrm{mg} / \mathrm{d})$, the fully adjusted OR was 0.92 (95\% CI: 0.83 to $1.00 ; p=0.11)$. In a cohort of middle-aged Australian women, there was a weak, nonsignificant inverse association between nitrate intake and self-reported CVD-related complications.

\subsection{Do Australians Maintain A Mediterranean Diet (MedDiet) after Trial Completion? 1-Year Follow-Up of} The MedLey Trial

K.J. Murphy ${ }^{1}$, K.A. Dyer ${ }^{2}$, B.J. Hyde ${ }^{2}$, C.R. Davis ${ }^{2}$, J.M. Hodgson ${ }^{3,4}$ and R.J. Woodman ${ }^{5}$

1 School of Pharmacy and Medical Sciences, University of South Australia, Adelaide, South Australia, Australia

${ }^{2}$ School of Health Sciences, University of South Australia, Adelaide, SA, Australia

${ }^{3}$ Medical School, University of Western Australia, Perth, WA, Australia

${ }^{4}$ School of Medical and Health Sciences, Edith Cowan University, Perth, WA, Australia

${ }^{5}$ Flinders Centre for Epidemiology and Biostatistics, Flinders University, Bedford Park, SA, Australia

The MedLey trial demonstrated participants could maintain high adherence to a Mediterranean diet (MedDiet) for six months. The MedDiet resulted in improved markers of cardiometabolic health in comparison with habitual diet (control). We have now followed participants for an additional 12 months in order to assess MedDiet adherence, blood pressure (BP), BMI and triglycerides. Participants completed a food frequency questionnaire and a 15-point MedDiet adherence score (MDAS; higher score = higher adherence) was calculated. Home BP was measured over six days, BMI was assessed and plasma triglycerides were measured. Data were analysed using intention-to-treat LMEM with a group $x$ time interaction term comparing data at baseline and 4 and 18 months (12 months post-trial). At 18 months, the MedDiet group had a MDAS of $7.9 \pm 0.3$ which was lower than the 4-month score $(9.6 \pm 0.2 p<0.0001)$, but higher than baseline $(6.7 \pm 0.2 p<0.0001)$. The MDAS in the HabDiet group remained unchanged over the 18 month period $(p<0.0001)$. Blood pressure, BMI and triglycerides were not significantly different to baseline. These data show some MedDiet principles could be adhered to for one year after finishing the MedLey trial. However improvements in cardiometabolic health markers were not sustained, indicating further dietary support may be beneficial to maintain high adherence.

\subsection{The Effects of Dietary Polyphenols on Iron Status and CVD Risk Markers-A Systematic Review}

H. Speer ${ }^{1,2}$, S. Cottin ${ }^{3}$, M. Botek 2,4, D. Sergi ${ }^{5}$, A. McKeune 2,6,7, E. Georgousopoulou 2,6,8, D.D. Mellor $2,6,9$ and N. Naumovski $2,3,6$

${ }^{1}$ Faculty of Science and Technology, University of Canberra, Bruce, ACT, Australia

${ }^{2}$ Collaborative Research in Bioactives and Biomarkers (CRIBB) Group, University of Canberra, Bruce, ACT, Australia

${ }^{3}$ Division of Food and Drink, Abertay University, Dundee, UK

${ }^{4}$ Department of Natural Sciences in Kinanthropology, Faculty of Physical Culture, Palacký University, Olomouc, Czech Republic

${ }^{5}$ Nutrition \& Health Substantiation Group, Nutrition and Health Program, Health and Biosecurity, Commonwealth Scientific and Industrial Research Organisation (CSIRO), Adelaide, SA, Australia

${ }^{6}$ School of Rehabilitation and Exercise Sciences, Faculty of Health, University of Canberra, Bruce, ACT, Australia

${ }^{7}$ University of Canberra Research Institute in Sport and Exercise (UCRISE), University of Canberra, Bruce, ACT, Australia 
${ }^{8}$ Discipline of Nutrition-Dietetics, Harokopio University, Athens, Greece

${ }^{9}$ School of Life Sciences, Coventry University, Coventry, UK

Supplementation of dietary polyphenols may mitigate the potentially negative effects of a Western diet with respect to development of cardiovascular disease (CVD). Polyphenols, while possessing cardioprotective qualities, have been shown to reduce iron absorption, potentially increasing CVD risk. Thus, we aimed to elucidate the relationship between polyphenol consumption, CVD risk markers and iron status. Five electronic databases (CINAHL, Cochrane Library, PubMed, SCOPUS, and Web of Science) were searched according to the PRISMA 2015 guidelines. Only randomised, double-blind, placebo-controlled human trials investigating the effects of polyphenol supplementation on inflammatory markers and iron status were included. Three studies meeting the inclusion criteria encompassed 280 participants receiving between 0.27 and $761 \mathrm{mg} / 100 \mathrm{~g}$ of polyphenols from a variety of foods (olive oil, bilberry juice and cherries). Overall, a significantly positive effect was reported $(p<0.05)$ on lipid profile, inflammatory markers and antioxidant status. Major findings include an increase in high-density lipoprotein cholesterol $(p=0.018)$, accompanied by a decrease in C-reactive protein $(p=0.027)$ and interleukin-6 $(p=0.037)$. No changes in haemoglobin levels were reported in any of the studies $(p>0.05)$. In conclusion, despite the supplementation of polyphenols being of potential therapeutic benefit, larger, well-controlled clinical trials are needed to address the role of both polyphenols and iron status on CVD development.

2.14. Impaired Fasting Glucose versus Impaired 120-min Glucose in Prediabetes: Differences in Weight Loss Maintenance-A PREVIEW Substudy

R. Muirhead ${ }^{1}$, T. Kyhala ${ }^{2}$, S. Brodie ${ }^{1}$, F. Atkinson ${ }^{1}$, M. Fogelholm ${ }^{2}$, A. Raben ${ }^{3}$ and J. Brand-Miller ${ }^{1}$

${ }^{1}$ University of Sydney, Sydney, NSW, Australia

${ }^{2}$ Department of Food and Nutrition, University of Helsinki, Helsinki, Finland

${ }^{3}$ Department of Nutrition and Exercise Science, University of Copenhagen, Copenhagen, Denmark

The PREVIEW intervention study (www.previewstudy.com) is a randomised controlled trial comparing two diets and two exercise strategies in $~ 2500$ overweight and obese individuals with prediabetes. After eight weeks weight loss using a low calorie diet (LCD, Cambridge Weight Plan), participants losing $8 \%$ or more were eligible to participate in the $\sim 3$ year weight maintenance phase. We hypothesised that difference in the pathophysiology of isolated impaired fasting glucose (iIFG) vs. isolated impaired glucose tolerance (iIGT) would influence weight loss and rate of weight regain. In the Sydney cohort at baseline $(n=121), 36 \%$ had iIFG $(n=44)$ and $31 \%(n=38)$ had iIGT. There were no differences in percentage weight loss after eight weeks LCD (iIFG $11.7 \pm 2.2 \%$ vs. iIGT $11.6 \pm 1.9 \%$ ). However, at six months, participants with iIGT had regained significantly more body fat (\% initial body weight) than those with iIFG and the difference remained significant at 12 months $(-3.1 \pm 0.57 \mathrm{vs}$. $-4.9 \pm 0.65 \%$ from baseline, $p=0.007)$. The findings suggest that individuals with IGT have intrinsic characteristics that promote faster weight regain compared to those with IFG. To our knowledge, this is a novel finding with implications for personalised nutrition interventions.

\subsection{Carbohydrate Restriction in Midlife is Associated with Higher Incidence of Type 2 Diabetes among} Australian Women with and without Previous Gestational Diabetes: A Cohort Study

J. Rayner ${ }^{1}$, E. Gresham ${ }^{2}$, L. Ross ${ }^{1}$, A. Hodge ${ }^{3}$ and D. Schoenaker ${ }^{4}$

${ }^{1}$ School of Allied Health Science, Griffith University, Southport, QLD, Australia

${ }^{2}$ Health Intelligence Unit, Western New South Wales Local Health District, Bathurst, NSW, Australia

${ }^{3}$ Cancer Epidemiology and Intelligence Division, Cancer Council Victoria, Melbourne, VIC, Australia

${ }^{4}$ Centre for Behavioural Research in Cancer, Cancer Council Victoria, Melbourne, VIC, Australia

Low carbohydrate diets are increasingly popular, but may be nutritionally inadequate. We aimed to examine if carbohydrate restriction in midlife is associated with type 2 diabetes (T2DM) risk, and if 
this association differs by previous gestational diabetes (GDM) diagnosis. Dietary intake was assessed for 9689 women from the Australian Longitudinal Study on Women's Health in 2001 (aged 50-55) and 2013 (aged 62-67) via validated Food Frequency Questionnaires. Average long-term carbohydrate restriction was assessed using a Low Carbohydrate Diet score (highest quartile (Q4) indicating lowest proportion of energy from carbohydrates). Log-binomial regression was used to estimate relative risks (RR) and 95\% CIs. Between 2001 and 2016, 959 women (9.9\%) developed T2DM. Carbohydrate restriction was associated with T2DM after adjustment for sociodemographic and lifestyle factors (Q4 vs. Q1: RR 1.27 (95\% CI: 1.10, 1.48)), but this was attenuated when adjusted for BMI (1.10 (0.95, 1.27)). Carbohydrate restriction was associated with lower consumption of fruit, cereals and high-fibre bread, and low intakes of these food groups were associated with higher T2DM risk. Associations did not differ by history of GDM. Carbohydrate restriction (with low intakes of fruit and grain products) was significantly associated with T2DM incidence in mid-aged women, regardless of GDM history.

\subsection{Developing an Innovative Model of Diabetes Care: A Novel PhD Partnership between INSPIRO Community Health and La Trobe University}

E. Kennedy ${ }^{1}$, G. Smith ${ }^{2}$, B. Gordon ${ }^{1}$ and A. Forsyth ${ }^{1}$

${ }^{1}$ La Trobe University, Bundoora, VIC, Australia

2 INSPIRO Community Health, Lilydale, VIC, Australia

The Yarra Ranges community in Victoria, Australia has a high risk of diabetes exacerbated by the distance from and lack of access to services. The local community health provider, INSPIRO, is partnering with La Trobe University in an innovative industry $\mathrm{PhD}$ model to develop, implement and evaluate an innovative community-based model of care for people living with diabetes in the Yarra Ranges. A community-based participatory research approach will be used to inform the new model of care. This will include consultation with key stakeholders, a scoping review of existing models of care, and a community forum with consumer and other key stakeholders. The proposed model of care will employ an action cycle approach with early implementation of the proposed model. Ongoing stakeholder engagement and feedback will inform modifications to the model of care. This poster will highlight the role of an innovative industry-academic partnership in improving community health in the Yarra Ranges.

\subsection{Effects of Macronutrient Manipulation on Postprandial Metabolic Responses in Males at Risk of Cardiovascular Disease during Simulated Shift Work}

M.P. Bonham ${ }^{1}$, E. Kaias ${ }^{1}$, G.K.W. Leung ${ }^{1}$, R. Davis ${ }^{1}$, N. Eikelis ${ }^{2}$, C. Murgia ${ }^{1}$ and C.E. Huggins ${ }^{1}$

${ }^{1}$ Department of Nutrition, Dietetics and Food, Monash University, Melbourne, VIC, Australia

2 Iverson Health Innovation Research Institute, Swinburne University of Technology, Melbourne, VIC, Australia

Night time eating is associated with increased postprandial triglyceride response, reduced glucose tolerance and insulin sensitivity. This may be related to the increased risk of metabolic diseases observed in night shift workers. We aimed to investigate the postprandial metabolic responses to two isocaloric meals differing in macronutrient composition at night. In a randomised cross-over trial, 16 males at risk of cardiovascular disease (overweight/obese, high fasting triglycerides $(>1.7 \mathrm{mmol} / \mathrm{L})$ ) were fed two isocaloric meals $(2.7 \mathrm{MJ})$ at $00.00 \mathrm{hr}$ : a 'healthy' and a 'processed' meal, which differed primarily in total fat, saturated fat and total sugars content. Postprandial blood samples were collected for glucose, insulin (3-h) and triglycerides (6-h), and analysed as incremental area under the curve (iAUC). Postprandial glucose $(p=0.04)$ and insulin iAUC $(p=0.02)$ were significantly higher after consumption of the processed meal compared to the healthy meal. Postprandial triglyceride iAUC was not statistically different between the two meal types $(p=0.72)$. Our results contribute to the growing literature on the impact of meal timing and food choice at night on metabolic health. It emphasises 
the need to consider the role and effects of nighttime eating, when developing metabolic disease prevention strategies for shift workers.

2.18. Resistant Starch is Protective against Diabetic Nephropathy by Inhibition of Complement Activation in a Mouse Model of Diabetes

M. Snelson, S.M. Tan, D. Deliyanti, J.L. Wilkinson-Berka and M.T. Coughlan

Monash University, Melbourne, VIC, Australia

Complement activation occurs in diabetic nephropathy with the downstream complement component C5a activating the innate immune system, contributing to inflammation. Resistant starch (RS) may be nephroprotective, however the effects of RS on complement activation and the innate immune system have not been explored. Six week old nondiabetic mice (dbh), diabetic mice (dbdb) and $\mathrm{dbdb}$ mice on a regular chow diet supplemented with $25 \%$ RS (dbdbRS) were maintained for ten weeks. Twenty-four hour urine was collected for albumin and C5a measurement by ELISA. Kidneys were digested and enriched for leukocytes using Percoll gradient and flow cytometry was performed. Diabetes was associated with an increase in albuminuria $(28.0 \pm 6.5 \mathrm{vs} .411 .3 \pm 275.8 \mu \mathrm{g} / 24 \mathrm{~h}, p<0.001$, dbh vs. dbdb), which was reduced in diabetic mice receiving RS supplementation (411.3 $\pm 275.8 \mathrm{vs}$. $125.6 \pm 37.3 \mu \mathrm{g} / 24 \mathrm{~h}, p<0.01$, dbdb vs. dbdbRS). Urinary C5a excretion was increased by diabetes $(92.6 \pm 17.6$ vs. $1324.0 \pm 429.7 \mathrm{pg} / 24 \mathrm{~h}, p<0.001$, dbh vs. dbdb) and decreased by RS (1324.0 $\pm 429.7 \mathrm{vs.}$ $577.7 \pm 123.1 \mathrm{pg} / 24 \mathrm{~h}, p<0.05$, dbdb vs. dbdbRS) and infiltrating renal macrophages were more likely to be positive for C5aR with diabetes ( $4.8 \pm 3.9$ vs. $54.0 \pm 27.8 \%, p<0.001$, dbh vs. dbdb), which RS supplementation reduced ( $54.0 \pm 27.8$ vs. $11.7 \pm 4.2 \%, p<0.01$, dbdb vs. dbdbRS). These studies support the notion that RS is protective against renal disease via inhibition of complement.

\subsection{Metabolic Effects of Resistant Starch Type 2: A Systematic Literature Review And Meta-Analysis of Randomised Controlled Trials}

M. Snelson, J. Jong, D. Manolas, S. Kok, A. Louise, R. Stern and N.J. Kellow

Monash University, Melbourne, VIC, Australia

Published evidence exploring the effects of dietary resistant starch (RS) on cardiometabolic health is inconsistent. This review investigates the effect of RS type 2 (RS2) supplementation on body weight, satiety ratings, fasting plasma glucose, $\mathrm{HbA1c}$, insulin resistance and lipid levels in healthy individuals and those with overweight/obesity, metabolic syndrome (MetS) or type 2 diabetes mellitus (T2DM). Five electronic databases were searched for randomised controlled trials (RCTs) published in English between 1982 and 2017. Trials were eligible for inclusion if they reported RCTs involving humans where $\geq 1$ group received $\geq 10 \mathrm{~g}$ /day of RS2 for $\geq 1$ week, and measured body weight, satiety, glucose and/or lipid metabolic outcomes. Twenty RCTs involving 569 participants were included. RS2 supplementation significantly reduced serum triacylglycerol concentrations $(\mathrm{MD}=-0.11 \mathrm{mmol} / \mathrm{L} ; 95 \% \mathrm{CI}:-0.18,-0.04, p=0.002)$ in healthy individuals $(n=227)$ and reduced body weight (MD $=-1.29 \mathrm{~kg} ; 95 \% \mathrm{CI}:-2.40,-0.17, p=0.02)$ in people with T2DM $(n=90)$, but had no effect on appetite, fasting plasma glucose, HbA1c, HOMA-IR, total cholesterol, LDL-cholesterol or HDL cholesterol in any patient subgroups. Most studies had a moderate-high risk of bias, were of short duration and contained small sample sizes. High quality, long-term trials are required to determine the efficacy of RS2 supplementation for the dietary management of metabolic disease.

\subsection{Development and Validation of a Novel Prediction Tool (InsuTAG 2.0 ) for Type 2 Diabetes in General} Population: AusDiab Cohort Study

R.N. Thota ${ }^{1}$, K. Abbott $^{1}$, D. Magliano ${ }^{2}$, A. Owen ${ }^{3}$ and M. Garg ${ }^{1}$

${ }^{1}$ University of Newcastle, Callaghan, NSW, Australia

${ }^{2}$ Baker Heart and Diabetes Institute, Melbourne, VIC, Australia 
${ }^{3}$ Monash University, Melbourne, VIC, Australia

We have previously shown InsuTAG, a product of fasting insulin and triglycerides, predicts insulin resistance and metabolic syndrome. In this study we aimed to develop a tool based on InsuTAG for predicting type 2 diabetes (T2D) in the general population. InsuTAG ${ }_{2.0}$ was derived using the $\beta$-coefficients of baseline fasting insulin and triglycerides for people who developed T2D in the AusDiab study. Multiple logistic regression and receiver operator characteristic curves were used to assess T2D prediction ability of baseline InsuTAG, InsuTAG ${ }_{2.0}$ and HOMA-IR. From $n=5244$ participants, $n=175$ developed T2D by five years, and a further $n=165$ by 12 years, giving a total of $n=340(6.5 \%)$ developing T2D. After accounting for established risk factors, each one unit increase in baseline InsuTAG 2.0 was associated with a doubling of diabetes risk at 12 years (OR [95\% CI]: 2.04 [1.73, $2.40]$ ), and adding InsuTAG 2.0 to known risk factors improved the ability to predict T2D at five and 12 years $\left(R^{2}\right.$-change: $\left.0.033, p<0.001\right)$. Optimal cut-point (Insutag $\left.{ }_{2.0} \geq 1.67\right)$ for InsuTAG 2.0 gave the highest level of sensitivity for identifying T2D (5 year: $66.29 \%$; 12 year: $62.94 \%$ ) over other parameters (InsuTAG and HOMA-IR). InsuTAG 2.0 is a sensitive potential long-term predictor of T2D, which might compliment short-term predictors such as AUSDRISK tool.

\subsection{Effect of Time and Temperature on Storage Stability of Anthocyanin in Queen Garnet Plum Nectar}

M.C. Singh, W. Price and Y. Probst

University of Wollongong, Wollongong, NSW, Australia

Anthocyanins are pigments in fruits and vegetables, which help in biological activities improving health and warding off diseases. In this context stability of anthocyanins in Queen Garnet Plum (QGP) nectar was assessed. The QGP (Prunus salicina) —Japanese hybrid plum-was processed to nectar and stored at $-20^{\circ} \mathrm{C}$. Analyses were undertaken from mixed batches of nectar at storage temperatures, $-20{ }^{\circ} \mathrm{C}, 4{ }^{\circ} \mathrm{C}$ and $25^{\circ} \mathrm{C}$ for a period of eight days. The Association of Official Analytical Chemists $\mathrm{pH}$ differential methods for total monomeric anthocyanin pigment content of fruit juices was used to determine the anthocyanin composition at each temperature over time. The initial concentration was $40.30 \mathrm{mg} / \mathrm{L}$ of anthocyanins and reduced to $20.38 \mathrm{mg} / \mathrm{L}$ at $-20{ }^{\circ} \mathrm{C}$ and $6.86 \mathrm{mg} / \mathrm{L}$ at $4{ }^{\circ} \mathrm{C}$. A decrease in $49.43 \%$ and $82.98 \%$ was observed for storage at $-20{ }^{\circ} \mathrm{C}$ and $4{ }^{\circ} \mathrm{C}$, respectively, and complete degradation of anthocyanins was observed at $25^{\circ} \mathrm{C}$. This study indicated increase in degradation of anthocyanins as temperature increases and improved storage stability at $-20^{\circ} \mathrm{C}$. The speed of decline was almost double from $-20{ }^{\circ} \mathrm{C}$ to $25^{\circ} \mathrm{C}$. Predicted reasons include oxidation, larger vessel headspace, shape of the storage vessel, time or heterogeneity of nectar samples to cause the decline. Different storage temperatures may vary the speed of degradation.

\subsection{Garden to Plate-Skills Focused Gardening and Nutrition Project for Adults}

N. Ingram, V. Bobongie and F. Foulkes-Taylor

Foodbank WA, Perth, WA, Australia

"Garden to Plate" was developed in 2014 due to adult vegetable consumption being a low $6 \%$, as identified in the 2011-2012 Australian Health Survey. This initiative aims to provide people with knowledge, skills and confidence to grow their own vegetables. Working in partnership with local government, Garden to Plate is a series of 10 monthly workshops covering a range of gardening topics, also incorporating practical nutrition advice to improve vegetable consumption. Garden to Plate (G2P) offers adult learning in a social, informal, educational setting and seeks to improve the participants' knowledge and confidence in growing food. Participants learn the skills and know-how to grow and prepare fruits, vegetables and herbs and this can improve their access to a low cost, sustainable source of fresh produce. This unique, all-inclusive program held at community gardens, is suitable for people of all abilities, ages and backgrounds. Over 1100 attendances at 108 workshops have been recorded since its inception. The 2016 survey identified $88 \%$ of G2P participants greatly 
improved their gardening skills and confidence, $95 \%$ participants eating more fruit and $100 \%$ eating more vegetables over the past six months. The 2018 survey results will be available for the NSA conference in November.

\subsection{Australian Examples of Specific Food Security Strategies over the Last 30 Years}

B. Wood, L. Barbour and R. Lindberg

Right to food Coalition-Victorian Chapter, Melbourne, VIC, Australia

Australia's Right to Food Coalition (RtFC) exists to improve the mental and physical health and wellbeing of all Australians by working to ensure equitable access to nutritious food (www.righttofood. org.au). This bibliography provides specific retrospective examples of food security initiatives, and was conducted to inform the RtFC and build workforce capacity. Most initiatives were funded by five main stakeholders, ranging across Federal State \& Territory Governments, Local Governments, Not-for-Profit Organisations and tertiary institutions. Over time, these organisations have demonstrated commitment and support in national, state, and local settings. A key challenge to achieving equitable distribution of nutritious food is the overcentralisation of the commercial food chain system. Another challenge is the lack of Government development of enough upstream intersectoral policies to support food security across the nation. No Australian policies have so far been found which act directly on the specific human right to food and water security in perpetuity for the population. The widespread actions of local communities and citizens are crucial and this sector is yet to be fully examined. This document of specific strategies to address food security presents opportunities to learn from previous experience and to build successful initiatives in future.

\subsection{Developing, Inspiring and Connecting Leaders in Nutrition: 2018 Oceanic Nutrition Leadership Platform Course Summary}

\section{T.P. Wycherley}

University of South Australia, Adelaide, SA, Australia

Capacity development and ongoing support are necessary features of a strong nutrition workforce. The Oceanic Nutrition Leadership Platform (ONLP) course is a biennial seven-day-long course for earlyand mid-career individuals working in nutrition related fields. To date, more than 2200 individuals globally have participated in Nutrition Leadership Platform courses (Europe, Africa, South-East Asia, Middle-East, Latin-America) using similar training models. ONLP participants were selected from applications, by an independent committee and attended the course in July 2018. Course content focused on leadership, communication, team building, influencing policy, social responsibility and industry/public health relationships. A postcourse evaluation assessed participant satisfaction using a 0 (completely dissatisfied) to 100 (completely satisfied) scale. Twenty-two participants from five Oceanic countries (Tonga, Fiji, Cook Islands, New Zealand and Australia) completed the 2018 course. Participants represented academia/research (59\%), industry (9\%), hospital/health service (9\%), government $(18 \%)$ and nongovernment $(5 \%)$ sectors. Participants $(n=18)$, on average, rated their overall course experience as 92 (range: 54-100) and the overall course quality as 90 (range: 34-100). The ONLP 2018 course was predominately considered a success by participants. Importantly, the course cohort has joined the ONLP Network strengthening its capacity to positively influence food systems, nutrition related health outcomes and food security in Oceania.

\subsection{Retaining Antioxidant Qualities in Blanched Kale (Brassica Oleracea L. var. Acephala) by Optimising} Blanching Conditions Using Response Surface Methodology

E. Chijoff ${ }^{1}$, E. Beckett ${ }^{2}$ and Q. Vuong ${ }^{1}$

${ }^{1}$ School of Environmental and Life Sciences, University of Newcastle, Ourimbah, NSW, Australia

${ }^{2}$ School of Medicine and Public Health, University of Newcastle, Ourimbah, NSW, Australia 
In the raw state, kale has been identified as being high in antioxidants. Food composition tables rely on data obtained from raw kale; however, the antioxidant properties of kale are mainly attributed to phenolic compounds and ascorbic acid, which are known to be degraded by heat. Blanching is a mild thermal process, used as a cooking technique, or as an initial step for long-term storage. Excessive heat or application time may impact the antioxidant capacity of kale, although responses to different methods are yet to be profiled. Therefore, this study aimed to investigate the impact of different blanching conditions and methods on the antioxidant properties of blanched kale when compared to raw kale. Optimal conditions to retain total phenolic compounds for hot water and microwave blanching (time, temperature/power) were identified using response surface methodology. Optimal blanching conditions were found to be $150 \mathrm{~s}$ at $69^{\circ} \mathrm{C}$ for hot water, and $24 \mathrm{~s}$ at $960 \mathrm{~W}$ for microwave blanching. Under optimised conditions, levels of phenolic compounds, vitamin $C$ and antioxidant capacity were either not significantly different $(p>0.05)$, or were significantly greater $(p<0.05)$, compared to raw kale. These results demonstrate how type specific blanching optimisation can maintain levels of antioxidant phytochemicals in kale.

\subsection{Phytochemical Composition of Australian Grown Opuntia Ficus Indica Cladode Juice and Pomace}

C.A. Gouws ${ }^{1,2}$, E. Georgousopoulou 1,2,3, D.D. Mellor ${ }^{1,2,4}$ and N. Naumovski ${ }^{1,2}$

${ }^{1}$ Nutrition and Dietetics, University of Canberra, Bruce, ACT, Australia

${ }^{2}$ Collaborative Research in Bioactives and Biomarkers (CRIBB) group, Bruce, ACT, Australia

${ }^{3}$ Nutrition and Dietetics, Harokopio University, Athens, Greece

${ }^{4}$ School of Life Science, University of Coventry, Coventry, UK

The Opuntia ficus indica (OFI) cacti is drought-resistant and known for its fruit: the Prickly Pear. The 'cladode' (cacti-leaf) is also edible but under investigated. The phytochemical composition of the commercially grown cladode as a cold-pressed juice and pomace (methanolic extraction $70 \%$ $v / v$ ) were analysed for titratable acidity (TA; \%), antioxidant characteristics, free radical scavenging activity (DPPH) and antioxidant capacity ((FRAP), Trolox equivalents (TE)) and bioactive composition, total phenolic (TP; Folin-Ciocalteu; Gallic acid equivalents (GAE)) and flavanol (TF; $\mathrm{AlCl}_{3}$; Catechin equivalents (CE)) content. Descriptive statistics and differences (independent $t$-tests, assuming equal variances) between juice and pomace were determined via SPSS (v23; IBM). Juicing of the cladode yielded $31.62 \%$ juice, containing a TA of $0.365 \pm 0.065 \%$. The juice contained TP $1212 \pm 1069$ $\mu \mathrm{g}_{\mathrm{GAE}}$, TF $113 \pm 8.74 \mu \mathrm{g}_{\mathrm{CE}}$; DPPH $1064 \pm 12.2 \mu \mathrm{M}_{\mathrm{TE}}$, FRAP $867 \pm 57.7 \mu \mathrm{M}_{\mathrm{TE}}$ and vitamin $\mathrm{C}$ $0.193 \pm 0.216 \mathrm{mg}$, whereas the pomace contained TP $54.17 \pm 0.210 \mu \mathrm{g}_{\mathrm{GAE}}$, TF $23.06 \pm 1.05 \mu \mathrm{g}_{\mathrm{CE}}$, $\mathrm{DPPH} 915 \pm 1.39 \mu \mathrm{M}_{\mathrm{TE}}$ and FRAP $433 \pm 3.79 \mu \mathrm{M}_{\mathrm{TE}}$. Significant differences $(p<0.001)$ between juice and pomace were observed for TP, TF, DPPH, CUPRAC and FRAP, where the juice contained consistently higher contents. Therefore, the composition of the cladode juice indicates that it's a good source of phytochemicals and highlights the potential for development of new food products.

\subsection{The Effect of Juice Processing on Phytochemical Content on Juice and Waste (Pomace) of Australian} Grown Prickly Pear Fruit (Opuntia Ficus Indica)

C.A. Gouws ${ }^{1,2}$, E. Georgousopoulou 1,2,3, D.D. Mellor ${ }^{1,2,4}$ and N. Naumovski ${ }^{1,2}$

${ }^{1}$ University of Canberra, Bruce, ACT, Australia

${ }^{2}$ Collaborative Research in Bioactives and Biomarkers (CRIBB) group, Bruce, ACT, Australia

${ }^{3}$ Nutrition and Dietetics, Harokopio University, Athens, Greece

${ }^{4}$ School of Life Science, University of Coventry, Coventry, UK

Prickly pear (PP) fruit are rich in phytochemicals but has a relatively short shelf-life. The use of 'on-farm' juicing techniques could increase the marketability of this fast-spoiling fruit. The effect of accessible juicing techniques on phytochemical composition of PP juice and pomace is relatively underinvestigated. White, orange and purple PPs fruits were juiced using a variety of accessible 
blenders and juicers. The composition of juice (neat) and pomace $(70 \% \mathrm{MeOH})$ were measured with respect to antioxidant characteristics, scavenging activity (DPPH; Trolox Equiv. (TE)) and antioxidant capacity (FRAP; TE); and bioactive content; Total Phenolic (TP; Folin-Ciocalteu; Gallic acid Equiv. (GAE)), flavanol (TF; AlCl3; Catechin equiv. (CE)) and betalain content (TB mg/100 mL). Differences were determined using one-way ANOVA (SPSSv23; IBM); significance was set at $p<0.05$. In juice, the highest levels were measured in stick-blended for TF (orange; $715.2 \mu \mathrm{g}_{\mathrm{CE}} ; p<0.001$ ), jug-blended for TP (purple; $3087 \mu \mathrm{g}_{\mathrm{GAE}} ; p<0.001$ ), cold-pressed for TB (orange; $3.31 \mathrm{mg} ; p<0.001$ ), DPPH (purple; $1178 \mu \mathrm{M}_{\mathrm{TE}} ; p<0.001$ ) and FRAP (purple; $29,900 \mu \mathrm{M}_{\mathrm{TE}} ; p<0.001$ ). In pomace, the highest contents were measured in cold-pressed for TP (white; $68.4 \mu \mathrm{g}_{\mathrm{GAE}} ; p<0.001$ ) and FRAP (orange; $495 \mu \mathrm{M}_{\mathrm{TE}}$; $p<0.001$ ). Findings indicate that cold-pressed juicing best preserved the overall phytochemical content and had the greatest juice yield, with pomace showing promise for potential product development.

2.28. Effects of Composition on Cooking And Texture Properties of Gluten-Free Pasta from Wholegrain Sorghum, Brown Rice and Chickpeas

\section{Stephanie, G. Fox, M. Gidley and N. Gunness}

Centre for Nutrition and Food Sciences, Queensland Alliance for Agriculture and Food Innovation, The University of Queensland, St Lucia, QLD, Australia

Sorghum is an important cereal but under-utilised for human consumption in Australia. It is gluten-free and rich in dietary fibres and polyphenols. Thus, it can have potential health benefits for coeliacs and help in the management of obesity and diabetes. Sorghum flour can be used in gluten-free food products, but has poor functional and textural qualities that can be improved by mixing with other flours and hydrocolloids. Six gluten-free pastas were prepared with wholegrain white (WSF) or red sorghum flour (RSF), brown rice flour, chickpea flour and guar gum (GG) with or without xanthan gum $(X G)$ at varying compositions. These pastas were evaluated for cooking and texture properties, and compared with three commercial pastas. The sorghum-based pastas had low cooking losses (4-5.17\%), and RSF pastas had significantly lower cooking losses than WSF pastas $(p \leq 0.05)$. Furthermore, they were significantly firmer, stickier and less cohesive than the commercial pastas $(p \leq 0.05)$. Similar chewiness was obtained for pastas made from RSF or WSF combined with GG and XG, and RSF with GG compared to the commercial wheat pasta. The development of gluten-free sorghum-based pasta with very good cooking properties is possible; however, textural characteristics should be further improved to meet the standards for high quality pasta.

\subsection{Usability Testing of the Home Food Environment Self-Assessment Tool (HEAT)}

\section{S.L. Williams and R. Shailer}

\section{Central Queensland University, North Rockhampton, QLD, Australia}

Few tools are available for self-assessment of home food environments. This study developed and tested the usability of the Home food Environment self-Assessment Tool (HEAT) that aims to assess aspects (discretionary foods, food storage and visibility, tableware size, mealtime structure and food rules) of home food environments that promote or hinder healthy food intakes and behaviours. The tool includes 34 questions with higher scores reflecting healthier environments. Twenty-four parents participated ( $96 \%$ female, age range: 25 to 54 years). Mean scores were discretionary foods $(6.3 \pm 3.0)$, food storage and visibility $(7.2 \pm 0.9)$, tableware size $(5.6 \pm 2.2)$, mealtime structure $(4.5 \pm 2.2)$ and food rules $(4.9 \pm 1.6)$. Most families stocked easily accessible discretionary foods, used medium- or large-sized glasses, cups and bowls; did not have dinner or breakfast together every day or have meals in a dining area; and did not have consistent rules regarding vegetable intake, snacking, eating locations or behaviours within the home. The System Usability Score (80.4) suggests HEAT is a useful tool that permits identification of aspects of home food environments that could be 
modified to better support healthy food intakes and behaviours of families. Further development and validity testing of HEAT is warranted.

2.30. Investigating the Production of Spray-Dried Ferrous Sulphate Microcapsules Using Transglutaminase Cross-Linked Protein to Fortify The Infant Formula

S. Yousefi, M. Sarvi and W. Weisany

Department of Agriculture and Food Science, Science and Research Branch, Islamic Azad University, Tehran, Iran

Approximately a quarter of the world's population is suffering from anaemia due to iron deficiency. Fortification of staple foods with iron is one of the best ways to deal with this issue. In our study, the ferrous sulphate (FS) was encapsulated by complex coating materials with maltodextrin (MD, DE6), whey protein isolates (WPI) and sodium alginate (SA). The transglutaminase enzyme (TGase) was used as cross-linking agent. The physicochemical properties and encapsulation efficiency (EE) of produced FS loaded microcapsules, developed by directly spray-dried microcapsule was investigated. Response surface methodology (RSM) was applied to optimise ferrous sulphate concentration (FS, 0.1-0.5 ppm), wall-to-core ratio (W/C, 5-9) and transglutaminase (TGase, 0.02-0.06 ppm) in the preparation of bioactive coating to develop the high-quality dried milk powder. Microcapsules prepared showed a high stability against oxygen and a low metallic taste, which subsequently resulted in constant release of FS. The optimised conditions for achieving the highest antioxidant activity $(34.80 \%)$, EE (89.34\%), yield (42.87\%) and porosity value (77.11\%); the lowest of the caking degree $(65.54)$ and thiobarbituric acid (TBA, 71.39) values were $0.2 \mathrm{ppm}$ FS, $8 \mathrm{~W} / \mathrm{C}$ and $0.04 \mathrm{ppm}$ TGase. Results revealed that the microencapsulation process is suitable for producing protein-based microcapsules containing sensitive ingredients.

\subsection{Gut Microbiota and Amylolytic Enzymes Change during In Vitro Fermentation of Native and Cooked} Wheat Starches with Varying Amylose Content

A.T. Bui ${ }^{1}$, B.A. Williams ${ }^{1}$, A.J. Slade ${ }^{2}$, E.C. Hoedt ${ }^{3,4}$, M. Morrison ${ }^{4}$, D. Mikkelsen ${ }^{1}$ and M.J. Gidley ${ }^{1}$

1 Centre for Nutrition and Food Science, The University of Queensland-QAAFI, St Lucia, QLD, Australia

${ }^{2}$ Arcadia Biosciences, Seattle, WA, USA

3 APC Microbiome Institute \& Department of Microbiology, National University of Ireland, Cork, Ireland

${ }^{4}$ Diamantina Institute, Faculty of Medicine, Translational Research Institute, The University of Queensland, Brisbane, QLD, Australia

A slower rate of starch digestion in the small intestine is associated with increased resistant starch (RS) entering the colon, thereby providing the potential to realise health benefits when fermented by the resident microbiota. One way to reduce digestion rate is to increase starch amylose (AM) content; however, mechanisms involved in gut microbial fermentation of high amylose RS substrates are largely unknown. In this study, a range of high AM wheat starches were extracted and treated to model native granular and cooked forms. These starches underwent in vitro fermentation using a porcine faecal inoculum. Fermentation data, in the form of gas kinetics and short-chain fatty acid profiles, revealed that the higher AM starches had slower fermentation kinetics, and that cooking has a larger impact on the fermentation kinetics of low AM starches. Four types of amylolytic enzyme activity were quantified in both the supernatant and microbial pellet, along with changes in diversity of key microbial species across the fermentation. This study highlights the impact of diverse high amylose wheat starches on resident microbiota and their amylolytic enzymes, providing information on the interactions involved during fermentation and increasing understanding of the potential health benefits of high AM wheat-based foods. 
2.32. Long-Term Paleolithic Diet Is Associated with Low Resistant Starch, Different Gut Microbiota Composition and Increased Serum TMAO Concentrations

A. Genoni ${ }^{1}$, M. Boyce ${ }^{1}$, C. Christophersen ${ }^{1,2}$, J. Lo $^{1}$, P. Lyons-Wall ${ }^{1}$, T. Bird ${ }^{3}$ and A. Devine ${ }^{1}$

${ }^{1}$ Edith Cowan University, Joondalup, WA, Australia

${ }^{2}$ Curtin University of Technology, Bentley, WA, Australia

${ }^{3}$ CSIRO, Adelaide, SA, Australia

The Paleolithic diet is promoted for improved gut health; however there is little evidence available to support these claims. A cross-sectional study evaluated the association between diet, gut microbiota and serum trimethylamine-n-oxide (TMAO) in long-term $(n=44,>1 \mathrm{yr})$ followers of a Paleolithic diet (PD) compared to controls $(n=47)$ consuming an Australian diet. Dietary intake was measured by 3-day records, microbial composition in stool samples (48-h) and serum TMAO using LC-MS. PD was stratified (Strict Paleolithic (SP) $(n=22)$ and Pseudo-Paleolithic (PP) $(n=22))$ based on adherence, specifically, exclusion of grains and dairy. Intake of resistant starch (RS) was lower in both Paleolithic groups compared to controls $(2.62,1.26$ vs. $4.48 \mathrm{~g} /$ day $(p<0.05))$; vegetable intake was higher in SP (6.68 vs. 3.83 serves/day, $p<0.01$ ), and there was reduced abundance of Bifidobacteria and Roseburia, but increased TMA-precursor Hungatella in Paleolithic groups $(p<0.05)$. Trimethylamine (TMA) is a gut-derived metabolite and forms TMAO, associated with CVD, and was higher in SP compared to PP and control $(p<0.01)$ and inversely associated with wholegrain intake $(r=-0.34, p<0.01)$. Although PD has been promoted for improved gut health, these results indicate that long-term lower wholegrain intake is associated with detrimental effects on gut microbiota and increased production of TMAO. A variety of fibre components may be required to maintain gut health.

\subsection{Exploring the Systemic Delivery of Short-Chain Fatty Acid (SCFA) in Healthy Humans}

P.A. Gill ${ }^{1,2}$, D. So ${ }^{2}$, M.C. van Zelm ${ }^{1}$, J.G. Muir ${ }^{2}$ and P.R. Gibson ${ }^{2}$

1 Immunology, Central Clinical School, Monash University and Alfred Hospital, Melbourne, VIC, Australia

2 Gastroenterology, Central Clinical School, Monash University and Alfred Hospital, Melbourne, VIC, Australia

Increasing systemic delivery of SCFA is associated with health benefits experimentally, but how this can be achieved in humans is poorly explored. The study aimed to determine whether delivery of SCFA to the circulation can be altered via fibre-associated colonic fermentation and direct absorption from foods and beverages. Healthy subjects $(n=10)$ consumed diets high $(38 \mathrm{~g} /$ day $)$ and low in fermentable fibre (18 g/day) in random order for 5 days with a 5 -day washout between and, in separate experiments, consumed $20 \mathrm{~mL}$ apple cider vinegar (10\% SCFA) after an overnight fast. SCFA concentrations in peripheral blood plasma were measured by gas chromatography before and after these interventions. Mean (SD) SCFA concentrations on day 5 increased from 57 (18) on the low fibre to 177 (50) $\mu \mathrm{M}$ on the high fibre diet ( $p<0.001$; paired $t$-test). Vinegar consumption increased SCFA concentrations from 121 (59) fasting to 264 (108) $\mu \mathrm{M}(p<0.05$; paired $t$-test) after $60 \mathrm{~min}$, with a subsequent return to baseline levels within $80 \mathrm{~min}$. Circulating SCFA concentration increase 3-fold via the dietary intake of fermentable fibre or oral SCFA-rich foods, providing dietary strategies that might additively lead to sustained increase in delivery of SCFA to the systemic circulation.

\subsection{Knowledge and Attitudes toward Gut Health, Probiotics and Prebiotics in Australian Adults}

\section{S. Khalesi and S. Williams}

Physical Activity Research Group, Appleton Institute and School of Health, Medical and Applied Sciences, Central Queensland University, Rockhampton, QLD, Australia 
Evidence highlights relationships between gut health, probiotics, prebiotics and the prevention/treatment of chronic diseases. Few studies have investigated factors associated with use of these products. This study examined Australians knowledge of gut health and attitudes toward probiotics and prebiotics to understand needs for health education. In 2017, 1265 adults (53\% female) completed an online survey. Most respondents were current or previous users of probiotics $(58 \% \mathrm{knew}$ what 'gut flora' (65\%) and 'probiotics' (75\%) are, and identified yoghurt as a natural probiotic source $(77 \%))$. Only $34 \%$ of respondents knew what 'prebiotics' were. Among users of probiotics, only $32 \%$ reported daily intake. Most consumers (55\%) preferred food sources of probiotics/prebiotics compared to supplements (16\%). Many respondents reported using probiotics 'to maintain health' $(46 \%)$, or because they aligned with their 'own knowledge and belief' (39\%). Among nonconsumers (42\%), many were willing to try probiotics if recommended by a health professional $(44 \%)$. Main barriers to use included cost (68\%), perceived lack of need (58\%) and preference for natural food sources $(48 \%)$. These findings highlight relatively high levels of probiotics consumption and knowledge and a need for more education about their role in health, natural sources and correct use of probiotics and prebiotics.

2.35. The Effect of Sulphated Polysaccharide Seaweed Extract "UX" on the Gut Microbiome of Overweight and Obese Individuals: A Double-Blind Placebo-Controlled Trial

L.A. Roach ${ }^{1}$, P.C. Winberg ${ }^{2}$, D. Harmelin ${ }^{3}$ and B.J. Meyer ${ }^{1}$

${ }^{1}$ School of Medicine, SMAH, University of Wollongong, Wollongong, NSW, Australia

${ }^{2}$ Venus Shell Systems, Bomaderry, NSW, Australia

${ }^{3}$ Graduate School of Medicine, University of Wollongong, Wollongong, NSW, Australia

Seaweed extracts for gut health have not been well explored in human clinical trials. A double-blind placebo-controlled trial, of six week duration was undertaken, to test the effect of a sulphated polysaccharide seaweed extract (seaweed extract "UX") on the composition of the gut microbiome. A total of 64 overweight or obese individuals were randomised to either $2 \mathrm{~g}$ or $4 \mathrm{~g}$ dose of seaweed extract "UX" or a placebo. Faecal samples were taken at baseline and six weeks and analysed using 16S rRNA gene sequencing. Statistical analysis was conducted using Primer 6 with Permanova software, a permutational multivariate analysis of variance was used to test the difference in changes in composition and abundance of gut flora genera between group treatments. There was a significant difference in the change of gut flora composition and abundance between the two pooled active groups compared to the placebo group $(p<0.05)$. A similarity percentage analysis showed that over $90 \%$ of the differences found could be explained by 15 taxa. Notably, Bifidobacterium increased 3-fold in the active groups while Akkermansia increased 8-fold in the $4 \mathrm{~g}$ treatment group. This is the first study to demonstrate the prebiotic potential of specific algal extract "UX" in humans.

\subsection{Fermentation Profiles of Insoluble Dietary Fibres from Selected Fruits and Vegetables}

W. Widaningrum, B. Flanagan, B. Williams, D. Mikkelsen and M. Gidley

Queensland Alliance for Agriculture and Food Innovation (QAAFI), The University of Queensland, Brisbane/St Lucia, QLD, Australia

Many studies have investigated the fermentability and health effects of the soluble fraction of Dietary Fibres (DF) extracted from food plants, but few have investigated those from the insoluble fraction of DF. As almost all DF sources consist of mostly insoluble dietary fibre (IDF), studies to investigate fermentability and health benefits of naturally occurring DF contain both soluble and insoluble DF need to be conducted. In this experiment, substrates containing mostly IDF from fruits and vegetables (apple, banana, pear, baby spinach, celery and carrot) were isolated by mincing and washing processes to represent gastrointestinal digestion. The remaining IDF samples were studied using in vitro fermentation with a pig inoculum. All substrates were fermented extensively as reflected 
by their gas production, with apple and pear being the most rapidly fermented ( 202 and $183 \mathrm{~mL} \mathrm{~g}^{-1}$ respectively), followed by celery, carrot, spinach and banana, with the latter two behaving similarly to wheat bran $\left(74 \mathrm{~mL} \cdot \mathrm{g}^{-1}\right)$. The end-products of fermentation were primarily acetate, propionate and butyrate in proportions of 65:20:10 for all samples. The results indicate that these substrates containing IDF have potential to be considered as having prebiotics activity.

\subsection{Monitoring of Food Intake: A Survey on Nutritional Policies in Indonesian Hospitals}

D. Budiningsari ${ }^{1}$, S. Shahar ${ }^{2}$, Z.A. Manaf ${ }^{2}$ and S. Susetyowati ${ }^{1}$

${ }^{1}$ Gadjah Mada University, Indonesia, Sleman, DI Yogyakarta, Indonesia

2 Dietetics Programme, School of Healthcare Sciences, Faculty of Health Sciences, National University of Malaysia, Kuala Lumpur, Malaysia

Inadequate energy and protein intake among hospitalised patients are often reported. Continuous effort has been made to identify or screen patients at high risk of malnutrition; however, lesser emphasis has been made in monitoring and documentation of nutritional intake. This study aims to investigate the current policies of Indonesian hospitals in terms of monitoring food intake of hospitalised adult patients. A self-administered semistructured questionnaire was filled out by healthcare staffs, namely nurses, dieticians and food serving assistants in the wards. In addition, the interview with the heads of nutrition department of those six hospitals was conducted to verify the current policies of monitoring food intake as a part of nutrition care process. Most respondents (73\%) stated that regular monitoring and evaluation of patient food intake takes place, with $64 \%$ reporting that the task was conducted routinely or daily. All hospitals in the study, except two hospitals, have implemented policies that require the recording of patients' food intake. Accordingly, the majority of the healthcare professionals $(61.3 \%)$ corroborated the presence of such policy in their respective hospitals. Hospitals should provide protocols and guidelines among interdisciplinary professionals, which include the regular monitoring of food intake to reduce the risk of hospital malnutrition.

\subsection{Patients' Experiences with And Perceptions of Recommencing Feeding after Colorectal Surgery: A Qualitative Study}

M. Rattray ${ }^{1}$, A. Marshall ${ }^{2}$, B. Desbrow ${ }^{1}$ and S. Roberts ${ }^{3}$

${ }^{1}$ School of Allied Sciences, Griffith University and Gold Coast Health, Southport, QLD, Australia

${ }^{2}$ Menzies Health Institute Queensland and School of Nursing and Midwifery, Griffith University, Gold Coast, QLD, Australia

${ }^{3}$ Menzies Health Institute Queensland and School of Allied Health Sciences, Griffith University, Gold Coast, QLD, Australia

Many patients who undergo lower gastrointestinal surgery neither recommence feeding within timeframes outlined by evidence-based guidelines nor meet their nutrition requirements in hospital. The aim of this study was to explore patients' perceptions of recommencing feeding after colorectal surgery to determine areas of improvement to meet their needs and expectations. This qualitative study involved one-on-one, semistructured interviews with colorectal patients receiving postoperative care in an Australian hospital. Purposive sampling was used to ensure maximal variation in age, gender and procedural type. Interviews were audio recorded, with data transcribed verbatim before being thematically analysed. Emergent themes and subthemes were discussed by all investigators to ensure consensus of interpretation. Sixteen patients were interviewed (female, $56 \%$; age, $61.5 \pm 12.3$ years). Three overarching themes emerged from the data: (i) Patients make food-related decisions based on ideologies, experience and trust; (ii) patients appreciate the opportunity to participate in their nutrition care; and (iii) how dietary information is communicated influences patients' perceptions of and behaviours towards nutrition. Enabling postoperative colorectal patients to select from a range of liquid and solid foods from postoperative day one in conjunction with 
surgeons delivering clear, simple and encouraging dietary-related information may facilitate patient participation in care and increase oral intakes.

2.39. Compliance to Oral Nutritional Supplements (ONS) among Elderly Patients Admitted at St. Luke's Medical Center, Quezon City, Philippines

M.F.M. Liwag-Atienza ${ }^{1}$, D.C.D. Redondo-Samin ${ }^{2}$, J.F. Inciong ${ }^{2}$ and M. Ramos Jr. ${ }^{3}$

${ }^{1}$ Department of Geriatrics, St. Luke's Medical Center, Quezon City, Metro Manila, Philippines

${ }^{2}$ Clinical Nutrition Services, St. Luke's Medical Center, Quezon City, Metro Manila, Philippines

${ }^{3}$ Department of Geriatrics, St. Luke's Medical Center, Quezon City, Metro Manila, Philippines

One main reason for malnutrition in the elderly is suboptimal or poor dietary intake. To augment the inadequacy of their nutrient intake, oral nutritional supplements (ONS) is usually prescribed in the hospital. The study aimed to document compliance and tolerance of ONS among hospitalised elderly patients. This observational study included 36 patients prescribed with oral diet and ONS with 1:1 dilution. Actual intake of ONS was monitored using food record form for three days and subjects were grouped into (1) consumed $>50 \%$ prescribed ONS (2) consumed $<50 \%$ prescribed ONS. Results showed that $78 \%$ of the elderly patients consumed $<50 \%$ of the prescribed volume of the ONS. Average compliance to ONS was $22 \%$ only citing dislike of taste as the most common reason for low or noncompliance. Prescribed ONS is observed to be wasted with the patients consuming a mean ONS volume of $132.6 \mathrm{~mL} /$ day which equates to an average of $38 \%$ of prescribed ONS. Average unconsumed ONS amounts to $66 \%$ of what has been paid by patient for each served ONS. Adjustment to prescribed ONS volume may be recommended to optimise intake in the hospitalised elderly patients and prevent out-of-pocket wastage.

\subsection{Assessing Body Fluid Volume Status in Children with Nephrotic Syndrome Using Bioelectrical Impedance}

L.C. Ward ${ }^{1}$, S. Brantlov ${ }^{2}$, L. Jødal ${ }^{3}$, A. Lange ${ }^{4}$, R. Andersen ${ }^{4}$ and S. Rittig ${ }^{4}$

${ }^{1}$ The University of Queensland, St Lucia, QLD, Australia

${ }^{2}$ Clinical Engineering, Aarhus University Hospital, Aarhuus, Denmark

${ }^{3}$ Nuclear Medicine, Aarhus University Hospital, Aarhuus, Denmark

${ }^{4}$ Pediatrics and Adolescent Medicine, Aarhus University Hospital, Aarhuus, Denmark

There is a pressing need for a reliable and simple method to provide clinicians with essential information about body fluid volume status in children with nephrotic syndrome (NS). Bioelectrical impedance analysis (BIA) is a promising method but calculation of absolute body water volumes may be inaccurate in children with NS. This study examined, with the use of raw BIA parameters, resistance indices (RI), phase angle (PA), bioelectrical impedance vector analysis (BIVA), resistance (R) and reactance, $\mathrm{XC}$ and cell membrane capacitance $(\mathrm{CM})$ to assess volume status. Eight children with active NS and 38 healthy controls (HC) (2-10 y) were enrolled. Whole-body impedance measurements (Xitron 4200) were made at baseline $(n=$ all $)$ and at remission in NS $(n=5)$. Significant $(p<0.05)$ mean differences between NS and HC were found: RI (43.3 vs. $\left.28.6 \mathrm{~cm}^{2} / \Omega\right)$; PA ( 3.0 vs. $\left.5.3^{\circ}\right) ;$ BIVA $(\mathrm{R} / \mathrm{H}$, 356.9 and $\mathrm{XC} / \mathrm{H}, 18.4$ vs. $\mathrm{R} / \mathrm{H}, 579.4$ and $\mathrm{XC} / \mathrm{H}, 58.8 \Omega / \mathrm{cm})$ and $\mathrm{CM}(0.53$ vs. $0.82 \mathrm{nF})$. Change in impedance parameters were indicative of increased body water (oedema) in patients with active NS compared to both the healthy controls, and patients in remission. These observations inform treatment strategies to manage oedema such as dietary salt reduction.

\subsection{The Effect of A Two-Week Maternal Low FODMAP Diet on Human Milk Oligosaccharide Composition}

T.K. Harb, P.S.W. Davies and R.J. Hill

University of Queensland, South Brisbane, QLD, Australia 
The effect of maternal dietary fermentable carbohydrates, or FODMAPs, on human milk oligosaccharide (HMO) concentrations is currently unknown. HMOs play a key role in the establishment of the infant gut microbiome. Thirty mothers were randomised to either a low FODMAP (LF) diet or a cow's milk and hen's egg-free (CMP/Egg) diet for two-weeks. Eliminated foods were reintroduced over one week following the intervention period. Breast milk samples were collected before, during and after the intervention period. The samples were analysed for changes in selected HMOs across the study period using high-performance liquid chromatography with tandem mass spectrometry. A linear mixed model analysis was performed to determine the trends in changes in HMO concentration between diet groups over time. No significant differences between the diet groups were found, but a trend towards significance $(p=0.092)$ was found for one HMO: lactodifucotetraose (LDFT). LDFT concentration was reduced during the intervention period and increased during the reintroduction period in the LF group. This pattern was reversed in the CMP/Egg group for the reintroduction phase. The reduction in LDFT concentration in breast milk found here for the mothers consuming the LF diet may have the potential to impact the infant gut microbiome and hence functionality.

\subsection{Breastfeeding and Respiratory Outcomes in the First Year of Life: A Secondary Analysis of 3 Cohort Studies}

S.M. Harvey ${ }^{1}$, V.E. Murphy ${ }^{1}$, P.G. Gibson ${ }^{2,3}$, J. Mattes ${ }^{1,4}$, A. Collison ${ }^{1}$ and M.E. Jensen ${ }^{1}$

${ }^{1}$ Priority Research Centre Grow Up Well, University of Newcastle \& Hunter Medical Research Institute, Newcastle, NSW, Australia

2 Priority Research Centre for Healthy Lungs, University of Newcastle and Hunter Medical Research Institute, Newcastle, NSW, Australia

${ }^{3}$ Department of Respiratory and Sleep Medicine, John Hunter Hospital, Newcastle, NSW, Australia

${ }^{4}$ Respiratory Department, John Hunter Children's Hospital, Newcastle, NSW, Australia

The protective role of breastfeeding on infant wheeze or bronchiolitis remains equivocal, particularly when the mother has asthma. Data from 3 cohort studies of maternal asthma in pregnancy conducted between 2007-2018 in Newcastle, Australia, were analysed. Mother-infant dyads were followed for 12 months. Mothers completed a validated questionnaire at six months $(n=624)$ and 12 months $(n=507)$ postpartum. Information on breastfeeding duration, exclusivity and respiratory outcomes were collected. Eighty six per cent of mothers initiated breastfeeding, $43 \%$ of infants were breastfed, whether partially or exclusively, to 6 months and 31\% of infants were receiving breast milk at 12 months. Wheeze was reported for $40 \%$ of infants at 6 months and $52 \%$ at 12 months. Bronchiolitis was reported for $19 \%$ of infants at 6 months and $34 \%$ at 12 months. Breastfeeding at least 6 months compared to never breastfeeding was associated with a reduced risk of wheeze (adjusted risk ratio (aRR) 0.50, 95\% CI: 0.28-0.90, $p=0.02$ ) and bronchiolitis (aRR0.47, 95\% CI: $0.24-0.92, p=0.028$ ) at 6 months. This effect was not seen at 12-month follow-up. Breastfeeding for the first six months of life was associated with a significantly reduced risk of developing infant wheeze and bronchiolitis. A larger sample size is needed to determine whether longer duration breastfeeding is beneficial in preventing adverse respiratory outcomes in infants in the first year of life.

\subsection{Commercial Growing up Milks: Comparing Content, Price and Intake Recommendations with Cow's Milk}

B. Hudson ${ }^{1}$, T. Worsley ${ }^{1}$ and Jane Willcox ${ }^{2}$

${ }^{1}$ Deakin University, Geelong, VIC, Australia

${ }^{2}$ La Trobe University, Melbourne, VIC, Australia

Commercial toddler or growing-up milks (GUMs) are a multibillion dollar industry and have potential to change children's eating patterns however studies on GUMs are sparse. This study aimed to compare the nutrient composition, costs and serving recommendations of GUM and cow's milk 
(CM). Twelve GUMs commonly available in major Australian supermarkets and pharmacies were compared with CM for energy, macro- and micronutrient content, recommended daily serves and cost. GUMs (mean per $100 \mathrm{~mL}$ ) had similar energy ( $287 \mathrm{~kJ}$ versus $293 \mathrm{~kJ})$, lower protein $(-33.1 \%)$ and fat $(-22.3 \%)$ and higher carbohydrate $(134 \%)$ contents in comparison to CM. No consistency in GUM formulation was apparent and additional ingredients in some GUMs included docosahexaenoic acid, lutein and galactooligosaccharides. Marked differences were seen in micronutrient profiles with GUMs being higher in vitamins $C$ and $D$ and iron, similar in calcium and sodium and lower in vitamins $A$, B6 and B12, iodine, biotin and trace elements than CM. GUMs were up to four times more expensive than CM per $100 \mathrm{~mL}$. GUMs maximum daily feeding recommendations ( $460 \mathrm{~mL}$ ) have potential to overfeed. This study is the first to compare GUMs and CM and signals the need for further research into GUMs contribution to children's diets and nutrition recommendations for GUM intake.

2.44. A Greater Intake of Medium-High Fat Dairy Foods during Pregnancy Is Not Associated with Increased Newborn Body Fatness

A. Meroni ${ }^{1,2}$, H.U. Dissanayake 1,2 , R. McMullan ${ }^{1,2,3}$, Y. Kong ${ }^{1,2}$, A. Gordon ${ }^{2,3}$, K.M. Mckenzie ${ }^{1,2}$, M. Phang ${ }^{1,2}$ and M.R. Skilton ${ }^{1,2}$

${ }^{1}$ Boden Institute of Obesity, Nutrition, Exercise \& Eating Disorders, The University of Sydney, Sydney, NSW, Australia

2 Sydney Medical School, The University of Sydney, Sydney, NSW, Australia

${ }^{3}$ Royal Prince Alfred Hospital, Sydney, NSW, Australia

The consumption of dairy foods during pregnancy is reported to be beneficial for both mothers and their babies, with most benefits deriving from low-fat dairy varieties. Previous studies showed that less than $30 \%$ of Australian pregnant women meet the Australian Dietary Guidelines (ADGs) for dairy (2.5 serves daily). A greater consumption of medium-high fat content dairy foods during gestation may influence foetal accretion of adipose tissue, a key aspect of cardiometabolic health. In this study, we assessed the intake of various dairy products in 158 pregnant women using a validated food frequency questionnaire. Newborn body fat percentage (BF\%) was measured using air displacement plethysmography. On average, mothers consumed 1.9 serves daily of dairy products, of which $75 \%$ were from medium-high fat varieties and only $24 \%(n=38)$ of women met the ADGs for dairy during pregnancy. Multivariable linear regression showed no significant associations between neither low nor medium-high fat dairy intake with neonatal $\mathrm{BF} \%(\beta=-0.03$ [95\% CI: $-0.16,0.10], p=0.65$ and $\beta=-0.03$ [95\% CI: $-0.10,0.03$ ] $p=0.35$ ). Our results suggest that Australian pregnant women may not be meeting the ADGs for dairy consumption. Additionally, no evidence was found that dairy foods are associated with neonatal body fatness.

\subsection{Preconception Maternal Erythrocyte Saturated to Unsaturated Fatty Acid Ratio Predicts Pregnancy after} Natural Cycle Frozen Embryo Transfer

B. Meyer ${ }^{1}$, C.C. Onyiaodike ${ }^{2}$, H.M. Murray ${ }^{3}$, R. Zhang ${ }^{3}$, F. Jordan ${ }^{4}$, A.E. Brown ${ }^{2}$, R.J.B. Nibbs ${ }^{5}$, H. Lyall ${ }^{2}$, N. Sattar ${ }^{4}$, S.M. Nelson ${ }^{2}$ and D.J. Freeman ${ }^{4}$

${ }^{1}$ University of Wollongong, Wollongong, NSW, Australia

2 School of Medicine, University of Glasgow, Glasgow, UK

${ }^{3}$ Robertson Centre for Biostatistics, University of Glasgow, Glasgow, UK

${ }^{4}$ Institute of Cardiovascular and Medical Sciences, University of Glasgow, Glasgow, UK

${ }^{5}$ Institute of Infection, Immunity and Inflammation, University of Glasgow, Glasgow, UK

The environment for embryo implantation and foetal growth and development is affected by maternal nutritional, metabolic and health status. The aim of this prospective cohort study was to test whether plasma metabolic and inflammatory biomarkers can predict pregnancy resulting from in vitro fertilisation (IVF). Women with a natural menstrual cycle undergoing frozen embryo transfer 
(FET) were recruited and fasting baseline blood samples were collected a mean of 3.4 days prior to the luteinising hormone (LH) surge and a nonfasting blood sample was taken on the day of FET. Ongoing pregnancy was defined by positive foetal heartbeat on ultrasound scan at day 45 post LH surge. Thirty-six pregnancies resulted from FET in 143 women. In an overall stepwise multivariable analysis, erythrocyte saturated to unsaturated fatty acid ratio was positively associated with ongoing pregnancy. A similar model incorporating day of FET covariates found that erythrocyte saturated to unsaturated fatty acid ratio, erythrocyte fatty acid average chain length and plasma log-triglycerides predicted ongoing pregnancy. In conclusion, a higher periconceptional saturated to unsaturated fatty acid ratio predicted ongoing pregnancy after natural cycle frozen embryo transfer and may reflect a maternal nutritional status that facilitates pregnancy success in this assisted conception scenario.

\subsection{Comparison of Multivitamin and Mineral Supplements for Australian Adolescents}

K. Aldwell ${ }^{1}$, H. Jebeile ${ }^{1,2}$, S.P. Garnett ${ }^{1,2}$, M.L. Gow ${ }^{1,2}$ and N.B. Lister ${ }^{1,2}$

${ }^{1}$ The Children's Hospital at Westmead, Institute of Endocrinology and Diabetes, Sydney

${ }^{2}$ Children's Hospital, Westmead Clinical School, The University of Sydney, Sydney, NSW, Australia

Adolescents are at risk of micronutrient deficiency including vitamin B6, vitamin A, iron, zinc, calcium, magnesium and phosphorus due to inadequate intake (National Nutrition and Physical Activity Survey 2011-12). Supplementation may promote nutritional adequacy but the most suitable products are unknown. This study evaluated the micronutrient content of commercially available multivitamin and mineral (MVM) products in Australia. The Australian Register of Therapeutic Goods was searched to identify adolescent appropriate MVM containing five or more vitamins/minerals available in Australian stores. Eligible products were scored based on number of micronutrients and, if include, the amount of 'at risk' nutrients (maximum score of 40). Top scoring (>30) MVM were compared to Recommended Dietary Intakes (RDI). Of the 76 identified, none provided adequate amounts of all at risk micronutrients. The top thirteen MVM met RDI for vitamin B6 and eight for vitamin A in females aged 13-18 and males aged 13 (none met RDI for males over 14). Only one met RDI for iron and two for zinc. None met RDI for calcium, magnesium or phosphorus. Overall, commercial MVM contain proportionately less minerals than vitamins. Some may be useful for supplementing sub-optimal intake in adolescents. Individual prescription of at risk nutrients may be required.

\subsection{Novel Urinary Biomarkers of Dietary Intake: Mapping the Literature}

E. Clarke, R. Williams, M. Rollo, K. Pezdirc and C. Collins

Faculty Health and Medicine, School of Health Sciences, The University of Newcastle, Newcastle, NSW, Australia

Dietary intakes are commonly assessed by established methods such as food frequency questionnaires (FFQ), food records or recalls. These self-report methods each have limitations that impact validity and reliability. Dietary biomarkers can provide objective verification of self-reported food and nutrient intakes, and is a rapidly evolving area. This scoping review aims to summarise novel urinary biomarkers of individual foods, food groups, dietary patterns or nutritional supplements that have been evaluated to date. Six key electronic databases were searched. Included studies were in healthy populations, published from the year 2000, and compared measured dietary intake to a urinary marker. Studies were excluded if they were in populations with medical conditions, measured nutritional requirements or bioavailability. Initial search identified 9985 studies, with 1036 full texts retrieved and 113 full texts included. Preliminary results indicate the most common dietary assessment methods used to evaluate urinary biomarkers were FFQs or food records. Of included studies, 59 used urinary biomarkers for individual foods, 48 on food groups, eight on dietary patterns and five on supplements. Identified biomarkers were for whole grains (alklyresorcinols), soy (daidzein, 
genistein) and fruit (proline betaine). While some novel urinary biomarkers have been identified, further validation studies are warranted to verify self-reported intakes.

\subsection{Are the Current Recommended Dietary Allowances for Iron Encourageing Iron Deficiency}

D.M. Frazer ${ }^{1,2}$ and G.J. Anderson 1,2,3

${ }^{1}$ QIMR Berghofer Medical Research Institute, Herston, QLD, Australia

${ }^{2}$ School of Medicine, The University of Queensland, St Lucia, QLD, Australia

${ }^{3}$ School of Chemistry and Molecular Biology, The University of Queensland, St Lucia, QLD, Australia

The recommended dietary allowance (RDA) for iron is calculated using estimates of the proportion of dietary iron absorbed. Currently, the amount absorbed is based on that of a person with normal functional iron levels but with minimal storage iron, as indicated by a serum ferritin (SF) of $15 \mu \mathrm{g} / \mathrm{L}$. However, the amount of iron absorbed from the diet is inversely regulated by body iron stores. Therefore, basing the RDA for iron on the absorption rate of an individual with a SF of $15 \mu \mathrm{g} / \mathrm{L}$ will encourage iron deficiency, as those with a higher SF will not absorb enough iron to replace obligatory losses. Body iron stores would fall until a SF of $15 \mu \mathrm{g} / \mathrm{L}$ is reached, at which point iron absorption would balance iron losses. Using recently published estimates of iron absorption based on a target SF of $70 \mu \mathrm{g} / \mathrm{L}$ in premenopausal women, we have recalculated the RDA for iron and found that it increases from $8 \mathrm{mg} /$ day to $15 \mathrm{mg}$ /day for adult males, from $18 \mathrm{mg} /$ day to $33 \mathrm{mg} /$ day for premenopausal women and from $8 \mathrm{mg} /$ day to $14 \mathrm{mg} /$ day for postmenopausal women. These estimates represent a significant increase in the RDA for each population examined and suggest that public health policy should be reevaluated to better reflect population iron requirements.

\subsection{Fasting or a Low Carbohydrate Diet Can Alter Body Iron Regulation: A Potential Novel Treatment for} Hereditary Hemochromatosis

D.M. Frazer ${ }^{1,2}$, C.S.G. Mirciov ${ }^{1,2}$, S.J. Wilkins ${ }^{1}$ and G.J. Anderson ${ }^{1,2,3}$

${ }^{1}$ QIMR Berghofer Medical Research Institute, Herston, QLD, Australia

2 School of Medicine, The University of Queensland, St Lucia, QLD, Australia

${ }^{3}$ School of Chemistry and Molecular Biology, The University of Queensland, St Lucia, QLD, Australia

Hereditary hemochromatosis patients develop iron loading due to the inappropriately low expression of the iron regulatory hormone hepcidin. Interventions that increase hepcidin production would, therefore, be beneficial in this condition. A recent study has demonstrated that gluconeogenic signalling in fasted mice can increase the expression of Hamp1-the gene encoding hepcidin. We have examined this effect in more detail to determine whether the pathway(s) responsible might provide novel targets for the treatment of disorders of iron homeostasis. To stimulate gluconeogenesis, wild type mice and $\mathrm{Hfe}^{-/-}$mice (a model of hereditary hemochromatosis) were fasted prior to euthanasia and hepatic Hamp1 expression determined. A low carbohydrate diet was also used to stimulate gluconeogenesis in $\mathrm{Hfe}^{-/-}$mice. Fasting was found to increase Hamp1 levels in both wild type and $\mathrm{Hfe}^{-/-}$mice when compared to unfasted animals. Preliminary results obtained from mice fed a low carbohydrate diet showed that stimulating gluconeogenesis in this manner can reduce the degree of iron loading in $\mathrm{Hfe}^{-/-}$mice. These results indicate that the induction of Hamp1 expression by gluconeogenic signalling is independent of HFE, and suggests that targeting the pathways involved could be of clinical benefit in patients with hereditary hemochromatosis.

2.50. Usual Dietary Anthocyanin Intake, Sources, and Their Impact on Blood Pressure in a Representative Sample of Australian Adults

E.O. Igwe ${ }^{1}$, K.E. Charlton ${ }^{1,2}$ and Y.C. Probst ${ }^{1,2}$

${ }^{1}$ University of Wollongong, Wollongong, NSW, Australia 
2 Illawarra Health and Medical Research Institute, University of Wollongong, Wollongong, NSW, Australia

Estimation of anthocyanin intake is an important preliminary step in understanding anthocyanin-health effects. This study aimed to determine anthocyanin intake, food sources and associations with measured blood pressure (BP) from the 2011-12 National Nutrition and Physical Activity component of the Australian Health Survey. This study was a secondary data analysis. Anthocyanin intake was calculated using an Australian anthocyanin database, usual anthocyanin intake modelled using the Multiple Source Method and food sources were determined by calculating contribution of food groups to total anthocyanin intakes. Regression analysis, adjusted for age, gender, BMI, smoking status and physical activity was used to assess the relationship between anthocyanin intake and BP in adults aged 50+ years. Mean intake of anthocyanin was $24.17 \pm 0.32 \mathrm{mg} / \mathrm{d}$ across age groups, berries were a commonly ranked top food contributor. There was an observed significant association between anthocyanin intakes and BP (systolic, $\beta=-0.04, p<0.01$ and diastolic, $\beta=0.01$, $p<0.01$ ). Seasonal berries make up the primary sources of anthocyanins in this population with intake inversely correlated with BP in older adults aged $50+y$. These results will be useful in assessing the relationships between anthocyanin intake and chronic diseases and hence facilitate progress in the anthocyanin-health research.

\subsection{To Study an Association between Micronutrient Dietary Supplement Usage And Blood Biomarkers in} Older Australians

K. Kaur ${ }^{1}$, C. Martin ${ }^{2}$, K. King ${ }^{1}$, S. Niblett ${ }^{1}$, M. Veysey ${ }^{1,3}$, M. Lucock ${ }^{2}$, M. McEvoy ${ }^{1,4}$ and E. Beckett ${ }^{1,4}$

${ }^{1}$ School of Medicine and Public Health, University of Newcastle, Newcastle, NSW, Australia

${ }^{2}$ School of Environmental and Life Sciences, University of Newcastle, Newcastle, NSW, Australia

${ }^{3}$ Hull-York Medical School, University of York, Heslington, York, UK

${ }^{4}$ Hunter Medical Research Institute, University of Newcastle, Newcastle, NSW, Australia

Dietary supplement usage is common among older adults as ageing increases risk of malnutrition. The relationships between micronutrient supplement usage and health remain unclear, particularly regarding cardiovascular diseases. In this study, the relationship between cardiometabolic biomarkers and micronutrient supplement usage was assessed in a large cohort of older Australians $(n=649)$, with the aim of further elucidating these relationships. Fasting blood samples were collected and serum lipids (low-density lipoprotein (LDL), high-density lipoprotein (HDL), triglycerides (TG) and total cholesterol (TC)) were assessed at the Hunter Area Pathology Service (HAPS) using standardised laboratory protocols. Statistical analyses were conducted using multivariable regression modelling (JMP v13) with adjustments for dietary and lifestyle variables and odds ratios (OR) and $p$-values $(p)$ were calculated. HDL was higher (OR 1.44, 95\% CI: 0.91-2.28, $p=0.004)$ whereas TG and TC:HDL ratio were lower among supplement users (OR 0.81, 95\% CI: $0.61-1.07$ and $0.88,95 \%$ CI: $0.75-1.04$, respectively; $p=0.041$ and 0.027 , respectively). Cholesterol and LDL were not significantly associated with supplement usage (OR 1.006, 95\% CI: $0.84-1.21$ and $0.99,95 \%$ CI: $0.80-1.21$, respectively; $p=0.427$ and 0.824 , respectively). The data suggests that micronutrient supplementation is associated with improved serum lipid profile. Further research is needed to determine if this translates into reduced cardiovascular disease risk.

\subsection{Mastication Behaviour is Associated with Both Satiation and Satiety}

D. Ni, S. Dhital, M. Gidley and N. Gunness

Centre for Nutrition and Food Sciences, Queensland Alliance for Agriculture and Food Innovation, University of Queensland, Brisbane, QLD, Australia 
Eating behaviour is known to be related with metabolic conditions such as obesity and diabetes. However the regulation of food intake is complex as it is affected by a range of psychological and physiological factors. This study focused on the satiation and satiety of common foods with wide range of texture, nutrient types and energy as well as mastication behaviours using 10 trained panellists. The relationships between satiation, satiety, quantity of food intake, energy intake, energy density, food digestion, mastication number and particle size distribution were explored. The results show that the mastication number, a typical index of eating habit, has a significant positive correlation with perceived post-meal satiety and significant negative correlations $(p<0.05)$ with perceived meal satiation as well as the quantity of food intake. In addition, compared to the food energy intake, mastication behaviour had stronger correlations with satiation and satiety perception. In conclusion, eating habits, especially the mastication number, not only affect the quantity of food intake, but also have a close relationship with perceived satiation and satiety.

\subsection{Vitamin D Use And Osteoarthritis in Australian Adults}

A. Owen, E. Zomer, J. Gilmartin-Thomas and I. Ackerman

Monash University, Melbourne, VIC, Australia

It has been suggested that vitamin $\mathrm{D}$ levels may be associated with the development and progression of osteoarthritis (OA), however randomised clinical trials of vitamin D supplements have failed to find evidence of benefit for OA pain or progression. Recent Australian clinical guidelines recommend against vitamin D use for OA management, although it is often marketed for 'musculoskeletal health'. This study undertook cross-sectional analyses of the 2014/15 Australian Bureau of Statistics National Health Survey (NHS) to examine vitamin D supplement use in adults with and without OA. Multivariate logistic regression was used to examine predictors of vitamin D use. Among 14,560 adults in the NHS, 15\% had self-reported OA. Use of vitamin D supplementation in the past fortnight was reported by $15.6 \%$ of those with OA, $26.1 \%$ of those with osteoporosis, and $7.3 \%$ of those without musculoskeletal disease. After adjustment for age, sex, osteoporosis, physical activity, smoking, diabetes, BMI and socioeconomic status, OA remained a significant predictor of vitamin D use $(p<0.001)$. This study highlights the need to gain a better understanding of the prevalence of vitamin $\mathrm{D}$ deficiency and supplementation decisions for those with $\mathrm{OA}$.

\subsection{Developing an Australian Food Composition Database for Flavonoids: Systematic Expansion to the} Flavan-3-ol Subclass of Foods

\section{Y. Probst and K. Lindsay}

\section{University of Wollongong, Wollongong, NSW, Australia}

Phytochemical intakes are associated with decreased risk of noncommunicable diseases though region-specific data are required. Currently no Australian food composition database exists. This study aimed to develop an Australian flavan-3-ol database. Adapted from a previous anthocyanin database, a systematic literature review was undertaken to source analytical Australian food data; initially trialed for catechins. Analytical data was evaluated following an established USDA method. Where published data was not obtained unpublished data was sourced or values were borrowed from the USDA or Phenol-Explorer databases. All data was aligned to the AUSNUT 2011-13 database. Australian analytical data (mg/100 g) was found for black tea (8.443 epigallocatechin (EGC), 7.238 epigallocatechin gallate (EGCG), 5.275 ECG, 8.858 theaflavin, 9.851 theaflavin gallate, 5.38 theaflavin $3^{\prime}$-gallate, 11.57 theaflavin digallate), green tea (46.11 EGC, 109 EGCG, 36.6 ECG, 0.26 theaflavin and 5.85 thearubigin), red wine (3.262 catechin, 1.165 epicatechin), apples (18.4 catechin, 50.05 epicatechin), barley ( 0.82 catechin), oats ( 0.79 catechin and 2.34 EGC) anise myrtle (1730 catechin) and lilly pilly (47 catechin and 290 epicatechin). The data was of moderate-low quality. The developed database will 
provide more accurate estimates of Australians flavonoid intakes. Additional analyses are required for Australian foods to decrease reliance on borrowed data and improve data quality.

\subsection{Stress Reduces Phosphorus Balance in Humans}

N. Serizawa ${ }^{1,2}$, M. Nishimuta ${ }^{1,3}$, N. Kodama ${ }^{2,3}$, M. Shimada ${ }^{3,4}$, Y. Yoshitake ${ }^{3,5}$, M. Ota ${ }^{1}$ and T. Yano ${ }^{1}$

${ }^{1}$ Graduate School of Food and Nutritional science, Toyo University, Ora-gun, GUNMA, Japan

2 Tokyo Shokuryo Dietitian Academy, Tokyo, Japan

${ }^{3}$ National Institute of Health and Nutrition, Tokyo, Japan

${ }^{4}$ Chiba Prefectural University of Health Sciences, Tokyo, Japan

${ }^{5}$ National Institute of Fitness and Sports in Kanoya, Kagoshima, Japan

Mental and physical stresses promote urine excretion of calcium (Ca) and magnesium (Mg). In this study, we examined the balance of minerals (such as sodium, potassium, $\mathrm{Ca}, \mathrm{Mg}$, phosphorus [P], zinc, iron, copper and manganese) in humans during exposure to several types of stress. Here, two metabolic studies of 15 days, including two successive balance periods of four days, were performed. The subjects were 23 healthy young women. During the balance periods, a 4-day rotating menu was served to the subjects. During the first balance period (stress period), half of the subjects were exposed to three types of stresses (i.e., cold exposure, calculation and restriction to a dark place), whereas during the second balance period (control period), they were not exposed to stresses. In a crossover manner, the remaining subjects were exposed to stresses during the second balance period. Balance is a value that excludes faecal and urine excretion from intake. We used IBM SPSS Statistics Version 24 for statistical analysis. The $\mathrm{P}$ balance was significantly lower during the stress period than during the control period. $(-0.60 \pm 1.51$ vs. $0.12 \pm 1.19 \mathrm{mg} / \mathrm{kg} \mathrm{BW} / \mathrm{d}, p<0.05)$. Thus, we demonstrated that mental and physical stresses reduce $\mathrm{P}$ absorption.

2.56. Comparison of Iodine Status Pre-and Postmandatory Iodine Fortification of Bread in South Australia Using Newborn TSH Concentration as A Marker: A Population Study

M. Wassie ${ }^{1}$, L.N. Yelland ${ }^{2,3}$, L. Smithers ${ }^{2}$, E. Ranieri ${ }^{4}$ and S.J. Zhou ${ }^{1}$

${ }^{1}$ School of Agriculture, Food and Wine, The University of Adelaide, Adelaide, SA, Australia

${ }^{2}$ School of Public Health, The University of Adelaide, Adelaide, SA, Australia

3 South Australian Health and Medical Research Institute, The University of Adelaide, Adelaide, SA, Australia

${ }^{4}$ South Australia newborn screening center, Women's and Children's hospital, Adelaide, SA, Australia

Mandatory iodine fortification of bread was implemented in October 2009 following a reemergence of iodine deficiency in Australia. This study aimed to compare the iodine status of south Australian pre- and postmandatory iodine fortification of bread using newborn thyroid stimulating hormone concentration (TSH) as a marker. Iodine deficiency in the population is indicated when more than 3\% of newborns have TSH $>5 \mathrm{mIU} / \mathrm{L}$. Newborn TSH data between 2005 and $2016(n=211,033)$ were extracted from the newborn screening program in South Australia. The percentage of newborns with TSH $>5 \mathrm{mIU} / \mathrm{L}$ was $5.1 \%, 6.2 \%$ and $4.6 \%$ in the prefortification (those born before October 2009), transition (born between October 2009 and June 2010) and postfortification (born after June 2010) groups, respectively. Newborns in the postfortification period had a 10\% lower risk [Incidence Rate Ratio (IRR), 0.90; 95\% confidence interval (CI): 0.87, 0.94] while newborns in the transitional period had a $22 \%$ higher risk [IRR, 1.22; $95 \%$ CI: 1.13, 1.31] of having TSH $>5$ mIU/L than newborns in the prefortification group. Using newborn TSH as a marker, South Australia remains mildly iodine deficient postfortification. Regular monitoring of iodine status using multiple indicators is important to evaluate the efficacy and safety of the fortification program. 


\subsection{How Much Sodium and Potassium Are Victorian Adults Consuming}

K.A. Bolton ${ }^{1}$, J. Webster ${ }^{2}$, K. Trieu ${ }^{2}$, J. Reimers ${ }^{3}$, S. Armstrong ${ }^{4}$, E. Dunford ${ }^{2}$, S. Jan ${ }^{2}$, M. Woodward ${ }^{2}$, B. Neal ${ }^{2}$, C. Nowson ${ }^{1}$ and C. Grimes ${ }^{1}$

${ }^{1}$ Institute of Physical Activity and Research (IPAN), Deakin University, Geelong, VIC, Australia

2 The George Institute for Global Health, University of New South Wales, Newtown, NSW, Australia

${ }^{3}$ Victorian Health Promotion Foundation (VicHealth), Carlton, VIC, Australia

${ }^{4}$ Heart Foundation, Melbourne, VIC, Australia

VicHealth is implementing a state-wide salt reduction initiative. This study assessed sodium and potassium intakes among Victorian adults and defined the main sources of sodium in the diet. A cross-sectional study of 338 adults provided a complete 24-h urinary collection in 2016/2017 and 151 completed a 24-h dietary recall. The mean age of participants was 49 years and $56 \%$ were females. Mean (95\% CI) 24-h urinary excretion was 130 (124-137) mmol/day for sodium and 76 (73-79) $\mathrm{mmol} /$ day for potassium. The urinary Na:K ratio was 1.82 (1.74-1.91). Mean 24-h intake estimated by diet recall was 116 (108-124) mmol/day for sodium and 94 (89-99) $\mathrm{mmol} /$ day for potassium. The Na:K ratio was 1.34 (1.23-1.46). Corresponding estimates for salt intake were 7.6 vs. $6.8 \mathrm{~g} /$ day. The leading dietary sources of sodium were bread products $(10 \%)$, cereal-based mixed dishes $(8 \%)$ and processed meats ( $8 \%)$. Most sodium derived from foods was obtained from retail stores $(58 \%)$, restaurants/takeaway outlets (17\%) and fresh food markets (12\%). Mean salt intake exceeded the maximum recommended intake of $5 \mathrm{~g} /$ day. Salt reduction programs will need to target diverse food types and multiple types of food outlets.

\subsection{Monitoring the Safety of Intense Sweeteners-An Added Sugars Replacement Dietary Exposure Model}

M. de Abreu, D. Mackerras and T. Hambridge

Food Standards Australia New Zealand, Canberra, ACT, Australia

Ten intense sweeteners (IS) are permitted in foods and beverages in Australia. To assess and monitor their safety in the food supply, their intakes are assessed against Health Based Guidance Values (HBGV). It is very resource intensive to collect IS use data. Therefore to focus limited resources a screening method to identify IS where exposures may exceed HBGVs was needed. A dietary exposure model assuming replacement of all added sugars with IS was conducted to determine population exposure to IS. Usual intakes of added sugars (mean and 90th percentile) from the 2011-12 NNPAS $(n=12,153)$ were modelled using the NCI method, and were divided by the relative sweetness of each IS and compared to the acceptable daily intake (ADI). Dietary exposure to IS based on the added sugar replacement method were below the ADI at the mean and 90th percentile for 2+ years male, and female population for eight of the 10 IS. Cyclamates exceeded the ADI at the mean and 90th percentile, and steviol glycosides at the mean. Exposure to the majority of IS assuming replacement of all added sugars are below the ADI. Further investigations of exposure to cyclamates and steviol glycosides are warranted.

\subsection{Nutrient Content of Fast Food Items Served by Fast Food Outlets in Canberra: A Pilot Study}

A. Haider, R. Jani, N. Naumovski, J. Kellett, V. Learnihan and R. Davey

University of Canberra, Moncrieff, ACT, Australia

Nearly two-thirds (63\%) of adults in Australia are overweight or obese. Australians consume energy-dense, nutrient-poor fast foods items with a consumption rate of three times a week, contributing greatly to obesity prevalence. A cross-sectional pilot study that aims to collect and compare the nutrient content of fast food items $(\mathrm{N}=239$ items) provided by fast food outlets in Canberra between 2015 and 2018 and identify the number of fast food items that have met national reference criteria of fat $(\mathrm{g})$, saturated fat $(\mathrm{g})$, sugar $(\mathrm{g})$ and sodium $(\mathrm{mg})$ content/100 g, provided by 
Food Standard Australia and New Zealand (FSANZ). Data were derived from fast food outlet official websites. Fast Food items were categorised into pizza, pasta, salad, side dishes and beverage and dessert food groups. The majority of nutrient content of the fast food items in Canberra have not changed significantly between the two-time points, with a consistent trend of high energy, high fat, saturated fat, sugar and sodium content per $100 \mathrm{~g}$. Moreover, $69 \%$ of the fast food items have not met national reference criteria for all nutrients examined. Substantial food menu reformulation is needed to improve the healthiness of fast food items available in Canberra.

\title{
2.60. Nutrition Safety Assessment of DHA from Canola
}

\section{R. Alhazzaa and R. Reuss}

\section{Food Standards Australia New Zealand, Majura Park, ACT, Australia}

DHA (22:6n-3) plays a role in a range of physiological functions and obtained from dietary sources such as fatty fish and marine oils. Recently, canola (Brassica spp.) has been genetically-modified (identifier: NS-B50027) to produce DHA in its seeds as a sustainable alternative to fish oils from wild fisheries. To permit the use of DHA canola oil for human nutrition in Australia and New Zealand, FSANZ conducted a comprehensive risk assessment. Published randomised controlled trials in humans were considered and found that DHA intake up to $6 \mathrm{~g} /$ day for 3 to 15 weeks did not raise safety concerns. This intake has been associated with decreases in blood total and LDL cholesterol, blood triglycerides, resting heart rate and blood pressure. Potentially adverse effects were inconsistently reported in a limited number of studies. As $6 \mathrm{~g} /$ day is above the $3 \mathrm{~g} /$ day Upper Limit (UL) for n-3 LC-PUFA, a cautious approach was adopted and dietary intake estimates were based on the total intake of DHA, DPA and EPA. Dietary intake estimates for all population groups in Australia and New Zealand were below the UL. We therefore concluded that the consumption of DHA canola oil will not pose a nutritional concern in Australia and New Zealand.

\subsection{Sodium Levels of Processed Meat in Australia: Supermarket Survey Data from 2010 to 2017}

\author{
E. Sparks ${ }^{1}$, K. Trieu ${ }^{1}$, C. Farrand ${ }^{1}$, C. Davidson ${ }^{2}$, E. Joldeski ${ }^{2}$, J. Reimers ${ }^{3}$ and J. Webster ${ }^{1}$ \\ 1 The George Institute for Global Health, Newtown, NSW, Australia \\ ${ }^{2}$ Heart Foundation, Melbourne, VIC, Australia \\ ${ }^{3}$ Victorian Health Promotion Foundation, Melbourne, VIC, Australia
}

High sodium intake increases blood pressure and consequently increases the risk of cardiovascular diseases. Worldwide, populations are consuming in excess of the maximum recommended amount of $2000 \mathrm{mg}$ sodium per day, and in Australia the best estimate is $3840 \mathrm{mg}$ sodium/day. In Australia, processed meats contribute approximately $10 \%$ of daily sodium intake to the diet. This study assessed the mean sodium levels of processed meats in 2010, 2013, 2015 and 2017, and calculated the percentage of products meeting the draft Australian Healthy Food Partnership (HFP) targets, from a systematic survey of the four major Australian supermarkets. A total of 2510 products were included. The mean sodium content of processed meats in 2017 was $857 \mathrm{mg} / 100 \mathrm{~g}$ (SD 470). There was no change in the mean sodium content of processed meats overall from 2010 to $2017(p=0.23)$, though there were significant reductions in bacon and sliced luncheon meats ( $p<0.001$ and $p=0.002$, respectively). In 2017, promisingly, $66 \%$ of products fell under the new draft HFP targets, however only $35 \%$ of products met their respective target. These results demonstrate the large potential for future reformulation efforts through the more stringent draft HFP targets, and evidence of technological feasibility.

\subsection{Updated Australian Food Composition Database (Formerly NUTTAB)}

S. Tompsett ${ }^{1}$, R. Sobolewski ${ }^{1}$, A. Craven ${ }^{1}$, D. Ballantyne ${ }^{1}$ and J. Cunningham ${ }^{2}$

${ }^{1}$ Food Standards Australia New Zealand, Majura Park, ACT, Australia

${ }^{2}$ Consultant to Food Standards Australia New Zealand, Canberra, ACT, Australia 
Food Standards Australia New Zealand (FSANZ) publishes Australia's reference nutrient database, NUTTAB. The database contains information on the levels of nutrients in a wide range of foods available in Australia. In October 2018, an updated version was published under a new name, The Australian Food Composition Database-Release 1. Release 1 is the most up to date reference nutrient database for use in Australia. The data is made available free of charge and may be useful for a wide range of work areas including nutrition labelling, research on diet and disease, education, food industry and to help consumers make better informed food choices. Release 1 contains nutrient data for 1533 foods and up to 245 nutrients per food. This release contains a number of enhancements since the publication of the previous database, NUTTAB 2010. These include easier identification of foods, a large amount of new data, a core set of nutrients for each food and enhanced functionality of the online searchable version of the database. Release 1 is available as both an online searchable database and downloadable files. The database and additional information are available on FSANZ's website at www.foodstandards.gov.au.

2.63. Plasma Phospholipid Long-Chain Omega-3 Polyunsaturated Fatty Acids Levels are Inversely Associated with Depressive Measures in Older Individuals

A. Alex ${ }^{1}$, K. Abbott ${ }^{1}$, J. Ferguson ${ }^{1}$, M. McEvoy ${ }^{2}$, P. Schofield ${ }^{2}$ and M. Garg ${ }^{1}$

${ }^{1}$ Nutraceutical Research Program, University of Newcastle, Callaghan, NSW Australia

${ }^{2}$ School of Medicine and Public Health, University of Newcastle, Callaghan, NSW, Australia

Dietary long-chain omega-3 polyunsaturated fatty acid (LCn-3PUFA) intake is thought to improve depressive symptoms. We examined the association between plasma phospholipid LCn-3PUFA composition and measures of depression in the Hunter Cohort Study. Plasma phospholipids were extracted and fatty acids measured by gas chromatography. LCn3-PUFA status was calculated as the sum of eicosapentaenoic and docosahexaenoic acids. Depression was measured using the Center for Epidemiological Studies-Depression Scale (CES-D). CES-D scores of 17 to 23 were classified as moderate depression and scores $\geq 24$ were classified as severe depression. Statistical analysis took age, sex and physical activity into account. A total of $n=1896$ participants (age: $66.0 \pm 7.4$ years; BMI: $28.8 \pm 4.9 \mathrm{~kg} / \mathrm{m}^{2}$; female: $n=943,49.7 \%$ ) were included. Eighty-seven (4.6\%) participants had moderate depression, while $n=101(5.3 \%)$ had severe depression. LCn-3PUFA levels were inversely correlated with CES-D scores $(r=-0.07, p=0.002)$. Higher LCPUFA was associated with a high odds ratio for low risk of depression (adjusted OR 0.94, 95\% CI: 0.89, 0.99). Participants with severe depression had significantly lower LCn-3PUFA than those without depression (6.70 \pm 2.38 versus $7.28 \pm 2.61, p=0.029$ ).

LCn-3PUFA status is inversely associated with depression in this sample of older individuals. Further research is required to determine whether older individuals will benefit from increased LCn-3PUFA consumption to reduce depression

\subsection{Association between Plasma Phospholipid Omega-3 Polyunsaturated Fatty Acids (LCn-3PUFA) and} Distress Symptoms in Older People

A. Alex ${ }^{1}$, K. Abbott ${ }^{1}$, M. McEvoy ${ }^{2}$, P. Schofield ${ }^{2}$ and M. Garg ${ }^{1}$

${ }^{1}$ Nutraceutical Research Program, University of Newcastle, Callaghan, NSW, Australia

${ }^{2}$ School of Medicine and Public Health, University of Newcastle, Callaghan, NSW, Australia

Psychological distress during ageing is associated with increased physical morbidity and mortality, decreased functional status and increased rate of dementia. We test the hypothesis that low level of circulating LCn-3PUFA is associated with increased levels of distress. This is a secondary analysis of older individuals in the Hunter Cohort Study. Plasma phospholipid fatty acid composition was measured by gas chromatography. LCn-3PUFA status was calculated by adding eicosapentaenoic and docosahexaenoic acids $(\%, w / w)$. Distress was measured using the Kessler Psychological Distress 
Scale (K10). K10 scores of 25 to 29 were classified as moderate distress; scores $\geq 30$ were classified as severe distress. Statistical analysis took BMI, age, sex, physical activity and diet into account. A total of $n=1896$ participants (age, $66.0 \pm 7.4$ years; BMI, $28.8 \pm 4.9 \mathrm{~kg} / \mathrm{m}^{2}$; female, $n=943$ or $49.7 \%$ ) were included in the analysis. Fifty-seven (3.0\%) participants had moderate distress while $n=36(1.9 \%)$ had severe distress. Adjusted and unadjusted analyses showed lower levels of LCn-3PUFA were associated with higher levels of distress (adjusted- $\beta$ [95\% CI]: 0.104 [ $-0.21,0.00], p=0.050$ ). Participants with moderate and severe distress had significantly lower LCn-3PUFA levels than those without distress $(6.55 \pm 2.75$ and $6.04 \pm 1.56$ versus $7.31 \pm 2.62, p<0.005)$.

This study shows an association between low circulating LCn-3PUFA levels and increased levels of distress in older individuals.

2.65. Vitamin D Status, Intake and Supplement Use: A Comparison of Retirement Village Residents and Privately Dwelling Elderly Australians

E. Beckett ${ }^{1,2}$, K. Kaur ${ }^{1}$, C. Martin ${ }^{3}$, Z. Yates ${ }^{4}$, R. Thota ${ }^{4}$, M. Garg ${ }^{4}$, J. Furst ${ }^{3}$, P. Jones ${ }^{3}$, M. Veysey ${ }^{1,5}$ and M. Lucock ${ }^{3}$

${ }^{1}$ Medicine \& Public Health, The University of Newcastle, Ourimbah, NSW, Australia

${ }^{2}$ HMRI, Newcastle, NSW, Australia

${ }^{3}$ Environmental \& Life Sciences, The University of Newcastle, Ourimbah, NSW, Australia

${ }^{4}$ School of Biomedical Science and Pharmacy, The University of Newcastle, Callaghan, NSW, Australia

${ }^{5}$ Hull York Medical School, University of York, York, UK

Vitamin D supplements are often recommended in the elderly as endogenous synthesis decreases with age. It is generally believed that retirement village residents enjoy more healthy lifestyles than those living privately. However, differences in vitamin D status, important for bone health and potentially related to risk for other diseases, between these two groups have not been assessed. We assessed circulating vitamin D (HLPC), vitamin D intake (food frequency questionnaire, supplement surveys) and prevalence of osteoporosis (self-reported), in an elderly cohort $(n=649)$ by living situation (50\% living in retirement villages). Average circulating vitamin D levels were higher in those living in retirement villages ( $95 \pm 2$ vs. $87 \pm 2 \mathrm{nmol} / \mathrm{L}, p=0.003)$ which remained significant when adjusted for BMI, age, gender, income, education and environmental UV levels prior to sample collection. However, prevalence of vitamin D inadequacy did not differ between groups $(p=0.7)$. Levels of vitamin D consumed via diet or supplements did not differ between groups $(p=0.3$ and $p=0.06$, respectively). Those with osteoporosis were more likely to take a vitamin $\mathrm{D}$ supplement if they lived in a retirement village (OR 2.3, 95\% CI: 1.2, 4.5, $p=0.01)$. These results may represent higher compliance to recommendations and increased UV exposure in those living in retirement villages.

\subsection{The Simplified Nutritional Appetite Questionnaire Is Associated with Quality of Life of People Living} with Dementia

N.M. D'Cunha ${ }^{1,2}$, S. Isbel ${ }^{1,2}$, E.N. Georgousopoulou ${ }^{1,2,3}$, J. Kellett ${ }^{1,2}$, M. Hunter ${ }^{1,2}$, A. Lahiouel ${ }^{2}$, A.J. McKune ${ }^{1,2}$ and N. Naumovski ${ }^{1,2}$

${ }^{1}$ Collaborative Research in Bioactives and Biomarkers (CRIBB) Group, Canberra, ACT, Australia

2 University of Canberra, Bruce, ACT, Australia

${ }^{3}$ Medical School, Australian National University, Canberra, ACT, Australia

Poor appetite has been identified as a common problem for older people that may impact their overall quality of life (QoL). In particular, people living with dementia (PLWD) with reduced appetite are at a greater risk for weight loss and nutritional deficiencies that can contribute to increased mortality. To investigate the relationship between appetite and QoL, the four-item Simplified Nutritional Appetite Questionnaire (SNAQ) and a 29-item health-related QoL questionnaire (DEMQOL) was administered to 24 PLWD (14 female) aged 84.7 years $( \pm 7.58)$ living in residential care or receiving respite day care. 
The mean SNAQ score was $15.6( \pm 1.97)$ out of a possible 20.0 indicating that participants were not at a significant risk of weight loss within the next six months. Mean score on the DEMQOL was 85.7 out of a possible 112 points representing moderate QoL. Results from a multiple linear regression revealed that increased appetite is associated with a higher QoL ( $\mathrm{B}=3.664,95 \% \mathrm{CI}: 1.38,5.95 ; p=0.003)$ independently of sex. The SNAQ is a simple tool that may be a useful instrument to monitor wellbeing and QoL for PLWD in aged-care settings. Confirmation of these results in larger prospective studies is needed.

2.67. Serum Vitamin D Concentrations Modulate Leukocyte Telomere Length, A Marker of Ageing and Age-Related Disease in Healthy Adults-A Systematic Review

\section{C.D. Ferraris}

\section{Celeste Ferraris, Balgowlah, NSW, Australia}

The anti-inflammatory and antiproliferative actions of Vitamin D may reduce attrition and turnover of leukocyte telomeres attenuating ageing and age-related disease. To determine if serum 25-hydroxyvitamin $\mathrm{D}(25(\mathrm{OH}) \mathrm{D})$ concentrations are associated with a modulation in leukocyte telomere length (LTL) in adult populations, a search in Medline Complete, CINAHL plus, Cochrane, PubMed and ScienceDirect generated six cross-sectional studies for review after exclusion and inclusion criteria were applied. Positive associations were found between higher serum $25(\mathrm{OH}) \mathrm{D}$ and longer LTL in four of the six articles, ranging from $p=0.001$ to 0.059 in significance after confounder-adjusted and -unadjusted models and stratification strategies. The research indicates maintaining serum $25(\mathrm{OH}) \mathrm{D}$ levels above $30 \mathrm{nmol} / \mathrm{L}$ but more widely $>50 \mathrm{nmol} / \mathrm{L}$, correlates significantly $(p=0.01)$ to increased LTL in middle-aged populations and that there is a dose-response relationship. The two studies in which no association was found are characterised by populations with either a singular younger age of $31 \mathrm{yrs}$ or an older mean age of $63.2 \mathrm{yrs}$. The research suggests optimising Vitamin D concentrations particularly in middle-aged populations is a possible avenue through which to modulate telomere related ageing and/or that middle-aged telomere attrition provides the platform for the increasing positive effect of Vitamin D on telomeres.

2.68. Modulating the Diurnal Metabolic Response: A Cross-Over Study Examining the Effectiveness of a High Protein Meal in Improving Postprandial Blood Glucose Control at Night

\section{R. Davis, C.E. Huggins, K. Nguo and M.P. Bonham}

Department of Nutrition, Dietetics, and Food, Monash University, Notting Hill, VIC, Australia

Night eating results in a relative hyperglycaemia, and could be a risk factor for type 2 diabetes in those who regularly eat at this time, such as shift workers. The aim of this study was to examine if a high protein meal attenuates postprandial glucose at night, compared with a standard protein meal. In a cross over design, ten healthy adults completed four acute meal challenges. Test meals were consumed at 8 am and $8 \mathrm{pm}$; high protein/low carbohydrate (HP/LC) meal (41\% of energy from protein, $29 \%$ from carbohydrates) or isocaloric standard meal (15\% from protein, $46 \%$ from carbohydrate). Bloods were sampled at fasting and 3hr postprandially. Freidman's test was conducted prior to post-hoc Wilcoxon sign test; Bonferroni corrected $p$-value $<0.0083$. Glucose median (IQR) iAUC after the HP/LC meal was similar to the standard meal when consumed in the morning (12.9 (19.9) vs. 36.4 (99.6) $\mathrm{mmol} / \mathrm{L} .3 \mathrm{hrs}, p=0.028)$. The HP/LC meal elicited a significantly lower glucose iAUC (59.6 (117.0) mmol/L.3hrs) compared to the control meal (208.8 (154.1) mmol/L.3hrs) when consumed in the evening $(p=0.005)$. A diurnal variation in response to the test meals was observed. An HP/LC meal is effective in lowering the postprandial glucose excursion observed with night eating, compared to the standard meal. 
2.69. Age Affects the Suppression of Appetite and Energy Intake by Protein-Rich Supplements

C. Giezenaar, I. Chapman, M. Horowitz and S. Soenen

Centre of Research Excellence in Translating Nutritional Science to Good Health, Adelaide Medical School, The University of Adelaide, Royal Adelaide Hospital, Adelaide, SA, Australia

Little is known about the appetite-suppressive effects of protein-rich supplements in older people. The study aimed to determine the effects of substitution, and addition, of carbohydrates and fat to whey protein on appetite, gastric emptying, glucose, gut hormones and energy intake in young and older men. In randomised, double-blind, order, 13 older $\left(75 \pm 2 \mathrm{yrs}, 26 \pm 1 \mathrm{~kg} / \mathrm{m}^{2}\right)$ and 13 younger (23 $\left.\pm 1 \mathrm{yrs}, 24 \pm 1 \mathrm{~kg} / \mathrm{m}^{2}\right)$ men ingested drinks $(450 \mathrm{~mL}$ ) containing protein/carbohydrate/fat: (i) $14 \mathrm{~g} / 28 \mathrm{~g} / 12.4 \mathrm{~g}\left(280 \mathrm{kcal} /{ }^{\prime} \mathrm{M}_{280}{ }^{\prime}\right)$; (ii) $70 \mathrm{~g} / 28 \mathrm{~g} / 12.4 \mathrm{~g}\left(504 \mathrm{kcal} /{ }^{\prime} \mathrm{M}_{504}{ }^{\prime}\right)$; (iii) $70 \mathrm{~g} / 0 \mathrm{~g} / 0 \mathrm{~g}$ (280 kcal/'P ${ }_{280}$ '); or (iv) $0 \mathrm{~g} / 0 \mathrm{~g} / 0 \mathrm{~g}\left(\sim 2 \mathrm{kcal} /{ }^{\prime} \operatorname{control}^{\prime}\right)$ on four study days. Appetite (visual analogue scales), gastric emptying (3D-ultrasonography), glucose, insulin, ghrelin, cholecystokinin and glucagon-like peptide-1 (GLP-1) concentrations (0-180 $\mathrm{min}$ ) and ad libitum energy intake (180-210 min) were determined. Results were analysed using ANCOVA. Suppression of energy intake by $\mathrm{P}_{280}$ compared to control was less in older (increase of $49 \pm 42 \mathrm{kcal}$ ) than younger (suppression of $100 \pm 54 \mathrm{kcal})$ men $(p=0.038)$. After all caloric drinks, GLP-1 concentrations increased more in older than younger men $(p<0.05)$. During the first phase of gastric emptying $(0-60 \mathrm{~min})$, ghrelin was suppressed less by $\mathrm{M}_{280}$ and hunger was suppressed less by control, $\mathrm{M}_{280}$ and $\mathrm{M}_{504}$, in older than younger men $(p<0.05)$. In conclusion, age affects the suppressive effects of protein-rich drinks on appetite, energy intake and gut hormones.

\subsection{Diet Quality Changes over 12 Years of Follow-Up in A Representative Sample of Australian Mid-Aged Women}

\section{J.K. Jackson, L.K. MacDonald-Wicks, M. McEvoy and A.J. Patterson}

University of Newcastle, Newcastle, NSW, Australia

The Australian population is ageing, placing it at increased risk of age-related disease. Poor diet is a leading risk factor for deaths in Australia, yet little is known about how population diet quality changes with increasing age. Women from the 1946-51 cohort of the Australian Longitudinal Study on Women's Health with complete food frequency questionnaire data for both 2001 and 2013 were included. Diet quality scores, including the Australian Recommended Food Score (ARFS, maximum score 74), the Mediterranean Diet Score (MDS, maximum score 17) and Nutrient Rich Foods Index (NRFI), were calculated. Wilcoxon matched pairs tests were used to detect changes in dietary intakes and diet quality over time. In 8161 mid-aged Australian women, small increases in diet quality were detected over 12 years using the ARFS (32.4 to 32.83; $p<0.0001)$ and the NRFI (78.4 to 86.8; $p<0.0001$ ), demonstrating intakes were more aligned with the Australian Dietary Guidelines and the Nutrient Reference Values in 2013. Overall MDS scores were low (6.7 to 6.6; $p<0.0001$ ), indicating poor alignment with a Mediterranean dietary pattern. Diet quality improved slightly among a representative sample of ageing Australian women over 12 years. There remains significant room for improvement in diet quality among Australian women.

\subsection{Reliability and Validity of MediCul (Mediterranean Diet and Culinary Index) in Older Australian Adults}

S. Radd-Vagenas ${ }^{1}$, M.A. Fiatarone Singh ${ }^{1,2}$, K. Daniel ${ }^{1}$, Y. Noble ${ }^{1}$, F. O'Leary ${ }^{3}$, Y. Mavros ${ }^{1}$, H. Brodaty ${ }^{4}$ and V.M. Flood ${ }^{1,5}$

${ }^{1}$ Physical Activity, Lifestyle, Ageing and Wellbeing Research Group, Faculty of Health Sciences, The University of Sydney, Lidcombe, NSW, Australia

${ }^{2}$ Hebrew SeniorLife and Jean Mayer USDA Human Nutrition Research Center on Ageing, Tufts University, Boston, MA, USA 
${ }^{3}$ Nutrition and Dietetics Group, School of Life and Environmental Science, Faculty of Science \& The Charles Perkins Centre, The University of Sydney, Camperdown, NSW, Australia

${ }^{4}$ Centre for Healthy Brain Ageing, University of New South Wales, Sydney, NSW, Australia

${ }^{5}$ Western Sydney Local Health District, Westmead Hospital, Westmead, NSW, Australia

A Mediterranean diet has been associated with multiple health benefits for chronic disease. Yet no index tool has been developed for, and/or validated in, a Western population to assess adherence to the 'traditional' dietary pattern and aspects of cuisine. We aimed to test the reliability and validity of the 50-item MediCul among older Australian adults. Participants were recruited January to March 2017 from the 45 and Up Study, as part of the Maintain Your Brain validation study, completing MediCul online on two occasions. Reliability was assessed using the intraclass coefficient (ICC) and Bland-Altman plots. Validity was tested against a 3-day food record (FR) using the Research Food Diary app. Participants $(n=84 ; 59.5 \%$ female) were aged 65.4 years $(S D=5.9)$ and overweight (BMI 26.1; $\mathrm{SD}=4.0)$. Mean MediCul scores at two time points $(\mathrm{n}=74)$ were 55.9/100.0 and 56.5/100.0, respectively. MediCul had very good reliability according to the ICC (ICC $=0.87,95 \%$ CI: 0.796, 0.914, $p<0.0001)$ and Bland-Altman plots. For validity, the Bland-Altman indicated MediCul overreported the score by 5.4 points versus FR, but with no indication of systematic bias $\left(\mathrm{y}=8.46-0.06^{*} \mathrm{x}\right)(95 \%$ CI: $-0.275,0.155, p=0.582$ ). MediCul has very good reliability and moderate validity for assessing adherence to a 'traditional' Mediterranean pattern among older adults.

\subsection{Vegetable Diversity, Injurious Falls and Fracture Risk in Older Women: A Prospective Cohort Study}

M. Sim ${ }^{1}$, L.C. Blekkenhorst ${ }^{1,2}$, J.R. Lewis ${ }^{1,2,3}$, C.P. Bondonno ${ }^{1,2}$, A. Devine ${ }^{1}$, K. Zhu 2,4, R.J. Woodman ${ }^{5}$, R.L. Prince ${ }^{2,4}$ and J.M. Hodgson ${ }^{1,2}$

${ }^{1}$ Edith Cowan University, WA, Australia

2 The University Western Australia, WA, Australia

${ }^{3}$ The University of Sydney, NSW, Australia

${ }^{4}$ Sir Charles Gairdner Hospital, WA, Australia

${ }^{5}$ Flinders University, SA, Australia

The importance of vegetable diversity for the risk of falling and fractures is unclear. Our objective was to examine the relationship between vegetable diversity with injurious falls and fractures leading to hospitalisation in a prospective cohort of older Australian women $(n=1429, \geq 70$ years). Vegetable diversity was quantified by assessing the number of different vegetables consumed daily. Vegetable intake was estimated using a validated food frequency questionnaire at baseline (1998). Over 14.5 years, injurious falls (events $=568,39.7 \%$ ) and fractures (events $=404,28.3 \%$ ) were captured using linked hospital records. In multivariable-adjusted Cox regression models, women with greater vegetable diversity (per increase in one different vegetable/d) had lower relative hazards for falls $(8 \% ; p=0.02)$ and fractures $(9 \% ; p=0.03)$. A significant interaction between daily vegetable diversity (number $/ \mathrm{d}$ ) and total vegetable intake $\left(75 \mathrm{~g}\right.$ serves $/ \mathrm{d}$ ) was observed for falls $\left(p_{\text {interaction }}=0.03\right.$ ) and fractures ( $\left.p_{\text {interaction }}<0.001\right)$. The largest benefit of higher vegetable diversity were observed in the one third of women with the lowest vegetable intake $(<2.2$ serves / $d$; falls HR $0.8395 \%$ CI: $0.71,0.98$; fractures HR $0.7495 \%$ CI: $0.62,0.89)$. Increasing vegetable diversity especially in older women with low vegetable intake may be an effective way to reduce injurious fall and fracture risk.

2.73. Effects of Exercise and Calcium-Vitamin D Fortified Milk on Quality of Life and Depressive Symptoms in Older Men: Secondary Analysis of A Randomised Controlled Trial

\section{S.J. Torres, J. Matthews, C.M. Milte, I. Hopkins, S. Kukuljan, C.A. Nowson and R.M. Daly}

Institute for Physical Activity and Nutrition, Deakin University, Melbourne, VIC, Australia

We have previously shown that a 12-month multicomponent exercise program, independent of calcium-vitamin- $\mathrm{D}_{3}$ supplementation, improved bone mineral density, muscle mass, strength and 
function in older men. Building on this work, the aim of this study was to investigate the effects of the intervention on health-related quality of life (HR-QoL) and depressive symptoms. This was a 12-month, factorial design randomised controlled trial in which 180 healthy men aged 50-79 years were allocated into four groups: exercise + fortified milk, exercise, fortified milk or controls. Exercise consisted of high-intensity resistance training with weight-bearing exercise $(3 \times 60-75 \mathrm{~min} /$ week $)$. Men assigned to fortified milk consumed $400 \mathrm{~mL} / \mathrm{d}$ of low-fat milk containing $1000 \mathrm{mg} / \mathrm{d}$ calcium and $800 \mathrm{IU} / \mathrm{d}$ vitamin- $\mathrm{D}_{3}$. HR-QoL and depressive symptoms were assessed using the SF-36 and Center for Epidemiologic Studies Depression Scale, respectively, at baseline and 6 and 12 months. There were no exercise-by-calcium-vitamin D interactions or main effects for exercise and calcium-vitamin D on any HR-QoL measure or depressive symptoms. This suggests that a multicomponent exercise program or calcium-vitamin D supplementation alone or in combination has no effect on health-related quality of life or depressive symptoms in healthy community dwelling older men, despite significant improvements in muscle and bone health.

\subsection{Modifying the Mediterranean Diet for An Australian Population: Cardiovascular and Cognitive Outcomes of the MedDairy Study}

A.T. Wade ${ }^{1}$, C.R. Davis ${ }^{1}$, K.A. Dyer ${ }^{1}$, J.M. Hodgson ${ }^{2}$, R.J. Woodman ${ }^{3}$, H.A.D. Keage ${ }^{1}$ and K.J. Murphy ${ }^{1}$

${ }^{1}$ University of South Australia, Adelaide, SA, Australia

2 Edith Cowan University, Perth, WA, Australia

${ }^{3}$ Flinders University, Adelaide, SA, Australia

A traditional Mediterranean diet does not meet calcium recommendations for older Australians, which may limit long-term sustainability. The MedDairy study examined whether a MedDiet supplemented with dairy foods could provide adequate calcium for older Australians, while improving markers of cardiovascular health, cognitive function and mood. A randomised controlled parallel cross-over design compared a MedDiet supplemented with 3 to 4 daily serves of dairy foods (MedDairy) against a low-fat control diet (LF). Forty-one participants with risk factors for CVD completed each dietary intervention for eight weeks. Home systolic blood pressure (SBP) was the primary outcome. Secondary outcomes included fasting glucose, insulin, lipids, cognitive function, psychological wellbeing and dietary compliance. Compared with LF, MedDairy increased calcium intake (mean difference $=1.0 \pm 0.2$ serves, $p<0.001$ ) and led to improvements in morning home SBP (mean difference $=-1.6 \pm 0.6 \mathrm{mmHg}, p=0.01$ ), triglycerides (mean difference $=-0.05 \pm 0.02 \mathrm{mmol} / \mathrm{L}$, $p<0.01$ ) HDL (mean difference $=0.04 \pm 0.01 \mathrm{mmol} / \mathrm{L}, p=<0.01$ ) and total cholesterol to HDL ratio (mean difference $=-0.4 \pm 0.10 \mathrm{mmol} / \mathrm{L}, p=<0.001$ ). Significant improvements were also observed for reaction time $(p=0.04)$ and self-reported $\operatorname{mood}(p=0.01)$. Our findings suggest that the MedDiet can be modified to include adequate calcium for an older Australian population while improving markers of cardiovascular health, cognitive function and psychological wellbeing.

\subsection{Proximal Colonic Crypt Hyperplasia is Reduced by Emu Oil in a Mouse Model of Colitis-Associated} Colorectal Cancer

L.C. Chartier ${ }^{1,2}$, K.E. Maiolo ${ }^{1,2}$, G.S. Howarth ${ }^{1,2,3}$, D. Trinder ${ }^{4}$, I.C. Lawrance ${ }^{4}$ and S. Mashtoub ${ }^{1,2,4}$

${ }^{1}$ Adelaide Medical School, The University of Adelaide, Adelaide, SA, Australia

${ }^{2}$ Gastroenterology, The Women's and Children's Hospital, North Adelaide, SA, Australia

${ }^{3}$ School of Animal and Veterinary Sciences, The University of Adelaide, Roseworthy, SA, Australia

${ }^{4}$ The University of Western Australia, Murdoch, WA, Australia

Ulcerative colitis is a chronic inflammatory bowel disease. Prolonged inflammation stimulates uncontrolled crypt lengthening, leading to colitis-associated colorectal cancer (CA-CRC) development. Emu Oil (EO), derived from the fat of the Australian Emu, has protected the intestine and promoted 
repair in preclinical models. We aimed to determine the impact of long-term EO treatment on colonic cell proliferation in normal mice and in a model of CA-CRC. Female C57BL/ 6 mice were injected with saline or azoxymethane $(7.4 \mathrm{mg} / \mathrm{kg}$ ) and underwent three dextran sulphate sodium/water cycles (DSS; $2 \% w / v)$. Mice were gavaged thrice weekly with water or EO. Colonic sections were stained with H\&E for quantitative measurements and immunohistochemically-stained (Ki-67) for proliferation. $p<0.05$ was considered significant. Colonic crypt depth and cell count increased in CA-CRC compared to normal controls $(p<0.01)$. Cell size and percentage of Ki-67 positive cells overall were unaffected $(p>0.05)$. Proximal colonic cell proliferation increased in CA-CRC controls and was attenuated by EO treatment. Distal colonic cell proliferation increased in the upper region of crypts in CA-CRC compared to normal controls. Crypt lengthening in CA-CRC is due to hyperplasia. EO attenuated proximal colonic cell proliferation in CA-CRC mice and did not affect intestinal growth in normal mice, suggesting its safety for long-term usage.

\subsection{Nutrition Focused Physical Examination: Using Simulation to Train Students and Practitioners}

M. Nahikian-Nelms, H.E. Doetsch, K. Garrison, R. Wehner, AM. Bittoni and K. Roberts

The Ohio State University, Columbus, $\mathrm{OH}$, USA

The purpose of this research was to determine the influence of an educational workshop using patient simulation on the perceived knowledge and confidence of dietetic students and practitioners in assessing malnutrition. A secondary aim of this research was to evaluate participants' ability to correctly diagnose malnutrition. After attending a classroom lecture on malnutrition, participants $(n=125)$ participated in a workshop which included (1) small group sessions on NFPE, (2) practice of NFPE on a partner and (3) application of NFPE and malnutrition diagnosis to simulated cases using trained actors. Instructors provided feedback following each simulation. Participants completed pre/post surveys on perceived knowledge and confidence in performance ability. A paired $t$-test evaluated changes in survey ratings. Descriptive statistics measured student accuracy of malnutrition aetiology and severity level. Perceived knowledge and confidence scores on all items, except for functional assessment, improved following the workshop $(p<0.001)$. After feedback, diagnostic accuracy of aetiology and severity level improved by $18 \%$ and $5 \%$ respectively. Perceived knowledge, confidence and ability to accurately diagnose malnutrition increased with hands on practice and feedback. Findings highlight the impact of an experiential workshop on teaching of the NFPE and provide direction for future training.

\subsection{Can Consumption of Nuts Improve the Nutritional Status of Older Adults at Risk for Malnutrition?}

S-Y. Tan ${ }^{1}$, S.L. Tey ${ }^{2}$ and R. Brown ${ }^{2}$

${ }^{1}$ Deakin University, Burwood, VIC, Australia

${ }^{2}$ Department of Human Nutrition, University of Otago, Dunedin, New Zealand

According to the United Nations, $12.5 \%$ of the world's population was aged 60 years and over in 2015. Older adults are at increased risk for malnutrition, which negatively impacts on their wellbeing, health, and quality of life. Improving nutritional intake is key to malnutrition prevention and management. This review explores the roles of nuts in improving the dietary intake of older adults. Although nuts have been shown to be effective in reversing malnutrition among children and pregnant women in developing countries, there was a distinct lack of research examining the role of nuts in elderly populations who are at risk of malnutrition. To date, the health effects of nuts have been demonstrated predominantly in individuals who are overnourished and at risk for metabolic diseases. The benefits of nuts among these populations were attributed to nuts' satiety-promoting properties and lower-than-expected energy availability, which suggests that nuts may be unsuitable for malnutrition management. However, modifications to the physical forms of nuts and considering variety and the timing of consumption may overcome these barriers, making nuts and nut products 
appropriate in improving the nutritional intake and health of older adults. This conceptual framework should be tested using intervention studies in the future.

\subsection{Characterisation of Fibres Using a Rapid In Vitro Fermentation Model}

D. So ${ }^{1}$, C.K. Yao ${ }^{1}$, N. Pillai ${ }^{2}$, R. Singh ${ }^{1,3}$, P. Gibson ${ }^{1,4}$ and J. Muir ${ }^{1}$

${ }^{1}$ Department of Gastroenterology, Monash University, Melbourne, VIC, Australia

${ }^{2}$ School of Engineering, RMIT University, Melbourne, VIC, Australia

${ }^{3}$ Centre for Technology Alternatives for Rural Areas, IITB-Monash Research Academy, Indian Institute of Technology Bombay, Mumbai, Maharashtra, India

${ }^{4}$ Department of Gastroenterology, Alfred Hospital, Melbourne, VIC, Australia

The clinical value of specific fibres depends partly on their fermentation characteristics, traditionally assessed by extended incubations in vitro. This study aimed to utilise a new model that rapidly and dynamically assesses fermentation over $4 \mathrm{~h}$ to compare fermentability of known and novel fibres. Fibres $(1 \mathrm{~g})$ were added to fresh faecal slurries from healthy participants $(n=3)$ and fermented for $4 \mathrm{~h}$ in chambers under anaerobic conditions. Substrates included known and the novel fibres, almond xylo-oligosaccharide (XOS-A) and sugarcane fibre (SCF). End points included total gas production and changes in $\mathrm{pH}$. Mean gas production over $4 \mathrm{~h}$ was greatest for fructo-oligosaccharide (FOS; $76 \pm 26 \mathrm{~mL} / \mathrm{g}$ ) followed by corn-derived XOS (xylo-oligosaccharide; $66 \pm 3$ ), inulin (47), XOS-A (26 \pm 8$)$, partially hydrolysed guar gum (16) and SCF ( $3 \pm 1)$. Differences were found for FOS vs. SCF ( $p=0.01$; uncorrected Fisher's LSD), XOS vs. SCF $(p=0.01)$ and FOS vs. XOS-A $(p=0.02)$. All fibres decreased faecal $\mathrm{pH}$ from baseline in proportion to gas production. This model confirmed that fibre chain length influences fermentability, but also clearly showed that fermentability of XOS depends on its source and SCF is minimally fermentable. Extension of the results to the spectrum of individual gases may provide additional insights into the behaviour of fibres in vivo.

\subsection{Effect of Whole Foods and Dietary Patterns on Markers of Subclinical Inflammation in Weight Stable Overweight and Obese Adults-A Systematic Literature Review}

\section{S. Cowan, E. Leeming, A. Sinclair, A. Dordevic, H. Truby and S. Gibson}

Monash University, Notting Hill, VIC, Australia

Reduction of subclinical inflammation is a potential target for chronic disease management. Adiposity is a known modifier of meta-inflammation; however the influence of dietary factors is less clear. This review examines the evidence from human trials evaluating effects of wholefoods or dietary patterns on circulating inflammatory markers in weight stable overweight and obese adults. It is the first review to investigate the effect of diet on inflammation, independent of changes in adiposity. This review was conducted using the Cochrane Collaboration Handbook for Systematic Reviews of Interventions, and data sources included Ovid MEDLINE, EMBASE, CINAHL and Cochrane. Quality of studies was evaluated using the Cochrane Collaboration's Risk of Bias Assessment tool. Twenty-eight studies were included assessing the effect of 17 different foods and dietary patterns over 38 inflammatory markers. Overall, wholefoods and dietary patterns were not found to have significant effects on inflammatory markers in weight stable overweight and obese adults. While whole grains, soy, dairy, citrus fruits, nuts and chocolate, ginger and high total antioxidant capacity diets showed potential to improve inflammatory profiles, inconsistent findings made it difficult to produce definite conclusions. Study design characteristics contributing to these inconsistencies are discussed and recommendations for future research in this area are presented. 


\subsection{Variation in Chain Length of the Saturated Fatty Acids Affects Postprandial Lipaemia}

N. Panth ${ }^{1}$, C. Dias ${ }^{1}$, K-J. Wynne ${ }^{2}$, H. Singh ${ }^{3}$ and M. Garg ${ }^{1}$

${ }^{1}$ Nutraceutical Research Program, University of Newcastle, Newcastle, NSW, Australia

${ }^{2}$ Department of Diabetes \& Endocrinology, University of Newcastle, Newcastle, NSW, Australia

${ }^{3}$ Riddet Institute, Massey University, Palmerston North, New Zealand

Saturated fatty acids of different chain lengths are metabolised differently, however, their effects on blood lipids are not clearly understood. This study aimed to investigate the impact of medium (MC-SFA) and long (LC-SFA) chain saturated fatty acids on postprandial lipaemia. After an overnight fast, healthy volunteers consumed biscuits containing $40 \mathrm{~g}$ of either butter (BB), coconut oil (CB) or lard (LB) in a randomised cross-over study with a minimum 7-day washout period between treatments. Blood samples were collected at baseline, 2, 3, 4 and $6 \mathrm{~h}$ postprandially and assessed for total cholesterol (TC) high-density lipoprotein cholesterol (HDL-C), low-density lipoprotein cholesterol (LDL-C) and triglyceride (TG). Sixteen participants (male/female, 8/8; BMI, $23.7 \pm 2.8$; age, $26.2 \pm 8.4$ ) completed the study. Postprandial TG response determined by area under the curve (AUC) following CB was $59.8 \%$ lower than after BB $(p<0.01)$ and $58.8 \%$ lower than LB $(p<0.01)$. The net AUC for LDL-C was significantly higher after $\mathrm{CB}$ compared to the $\mathrm{BB}$ consumption, despite no differences in net AUC for TC and HDL-C. Consumption of MC-SFA resulted in lower postprandial TG concentrations compared to LC-SFA suggesting that food source rather than saturated fat content determines their lipemic responses.

\subsection{Association between Nutritional Status and Mortality among Adult Patients with Chronic Kidney} Disease on Maintenance Haemodialysis in a Provincial Hospital in the Philippines

D.C.D. Redondo-Samin ${ }^{1,2,3,4,5}$ and C.S. Bernardo ${ }^{6}$

1 Discipline of Nutrition and Dietetics, Faculty of Health, University of Canberra, Bruce, ACT, Australian

${ }^{2}$ Department of Internal Medicine, Premiere Medical Center, Cabanatuan City, Nueva Ecija, Philippines

${ }^{3}$ Clinical Nutrition Services, St. Luke's Medical Center, Quezon City, Metro Manila, Philippines

${ }^{4}$ School of Nutrition, Philippine Women's University, Manila, Metro Manila, Philippines

${ }^{5}$ Department of Internal Medicine, Dr. Paulino J. Garcia Memorial Research and Medical Center, Cabanatuan City, Nueva Ecija, Philippines

${ }^{6}$ Department of Internal Medicine, Premiere Medical Center, Cabanatuan City, Nueva Ecija, Philippines

Prevalent among haemodialysis patients is malnutrition, which is an independent determinant of mortality. This study aimed to determine the association of nutritional status and mortality among haemodialysis patients in a provincial hospital in the Philippines. Twenty-nine haemodialysis patients recruited between 2010 and 2012 were included in this prospective study. Baseline nutritional status assessment including modified subjective global assessment, body mass index (BMI), serum creatinine and anthropometric measurements were analysed and mortality rate was followed up. Patients with below normal tricep skinfold (TSF), mid-arm circumference (MAC) and mid-arm muscle circumference (MAMC) had higher mortality rates. Increasing BMI is correlated with decreased mortality as no overweight patients died, while $35 \%$ with normal BMI and $42.8 \%$ who were underweight died, respectively. It was also observed that those with lower creatinine levels had higher mortality rates. Survival analysis showed that serum creatinine level of 10.1 to $15.01 \mathrm{mg} / \mathrm{dL}$ was associated with decreased risk of mortality $(p=0.046)$. None of the patients who had normal nutritional status died while 50\% with below normal nutritional status died within two years. Early assessment of the nutritional status is recommended to prevent the development or progression of malnutrition in haemodialysis which may decrease mortality rates. 
2.82. Evaluation of Nutritional Adequacy and Symptom Improvement during Implementation of the Low-FODMAP Diet in Individuals with Irritable Bowel Syndrome

K.M. Roberts, M.L. Nahikian-Nelms, K.S. Al-Muhanna, J. Clutter, K. Corfman and J. Richards

The Ohio State University, Columbus, OH, USA

This study aims to define the duration of the elimination phase for symptom relief and to assess the nutritional adequacy of a low-FODMAP diet. Subjects attended a dietician-led education class prior to initiation of a 6-week, low-FODMAP diet. Subjects completed IBS-Symptom Severity Scale questionnaires (IBS-SSS), diet assessments and dietary compliance questionnaires throughout the study. Complete data was available for 15 of 35 subjects. All subjects $(n=15)$ had a clinical response (reduction of 50 points on IBS-SSS) with the majority of symptom improvement between baseline and week two $(n=13 ; p=0.001)$. There was a statistically significant reduction in grams of carbohydrates $(p=0.031)$, grains $(p=0.048)$ and dairy $(p=0.010)$ while following a low FODMAP diet. Several micronutrients (calcium, iron, folate, vitamin C and vitamin D) were below the recommended dietary allowance for $>75 \%$ of subjects, although many of these inadequacies were present at baseline. The rate of noncompliance was high (71\%). Our findings support a two-week elimination of FODMAPs may be optimal for achieving symptom relief. The high dropout rate and poor compliance highlight the need to assess and report dietary compliance in future interventions to gain insight into the efficacy of a low FODMAP diet.

\subsection{An Anti-Inflammatory Meal Postexercise Augments the Exercise-Induced Reduction in Airway Inflammation in Adults with Asthma}

K. McDiarmid ${ }^{1,2}$, J.W. Upham ${ }^{3}$, L.G. Wood ${ }^{1}$, L. MacDonald-Wicks ${ }^{2}$, N. Shivappa ${ }^{4,5}$, J.R. Hebert ${ }^{4,5}$ and H.A. Scott 1,3

${ }^{1}$ Centre for Healthy Lungs, The University of Newcastle, New Lambton Heights, NSW, Australia

2 Nutrition and Dietetics, School of Health Sciences, The University of Newcastle, Callaghan, NSW, Australia

${ }^{3}$ Lung and Allergy Research Centre, The University of Queensland, Woolloongabba, QLD, Australia

${ }^{4}$ Cancer Prevention and Control Program, University of South Carolina, Columbia, SC, USA

${ }^{5}$ Department of Epidemiology and Biostatistics, Arnold School of Public Health, University of South Carolina, Columbia, SC, USA

Exercise appears to reduce airway inflammation in adults with asthma. Dietary intake also influences airway inflammation. The Dietary Inflammatory Index (DII) quantifies the inflammatory potential of the diet and has been associated with asthma outcomes. The aim of this study was to quantify the DII of a meal consumed postexercise and to establish whether this influences changes in airway inflammation in adults with asthma. Adults with stable asthma were randomised to intervention (30-45 min exercise on a cycle ergometer, $n=38$ ) or control (30 min rest, $n=18$ ) groups. A self-selected meal was consumed two hours postintervention; energy-adjusted DII was calculated and used to categorise participants into an 'anti-inflammatory' or 'pro-inflammatory' group. Airway inflammation (sputum eosinophils) was measured the day prior to and four hours postintervention. Following exercise, sputum eosinophils decreased $-0.5(-2.0,0.3) \%$ in the anti-inflammatory group and increased $0.5(0,3.0) \%$ in the pro-inflammatory group $(p=0.012)$. Conversely in the control group, meal DII had no impact on sputum eosinophil change $(p=0.845)$. The combination of an anti-inflammatory meal with exercise reduced sputum eosinophils compared with an anti-inflammatory meal without exercise $[0.5(0.3,2.5) \%, p=0.011)$. Consuming an anti-inflammatory meal two hours postexercise augments the anti-inflammatory effects of exercise in adults with asthma. 


\subsection{A Higher Omega-3 Index is Associated with Better Asthma Control}

I. Stoodley ${ }^{1,2}$, M. Garg ${ }^{3}$, H. Scott ${ }^{1,3}$, L. Macdonald-Wicks ${ }^{2}$, B. Berthon ${ }^{1,3}$ and L. Wood ${ }^{1,3}$

1 Priority Research Centre for Healthy Lungs, Hunter Medical Research Institute, Newcastle, NSW, Australia

2 School of Health Sciences, University of Newcastle, Newcastle, NSW, Australia

${ }^{3}$ School of Biomedical Science and Pharmacy, University of Newcastle, Newcastle, NSW, Australia

Asthma is a chronic inflammatory disease of the airways and is also associated with systemic inflammation. Long-chain omega-3 polyunsaturated fatty acids (LCn-3PUFA) have established anti-inflammatory effects, thus have the potential to be beneficial in asthma. The aim of this study was to determine the relationship between LCn-3PUFA and clinical asthma outcomes. Adults with $(n=217)$ and without asthma $(n=101)$ had blood collected. Lung function was measured and Juniper asthma control questionnaire (ACQ) scores were calculated. Fatty acids were measured in erythrocyte membranes by gas chromatography and the omega-3 index (O3I) calculated (\%eicosapentaenoic acid $+\%$ docosahexaenoic acid). A higher O3I was noted in subjects with controlled or partially controlled asthma (ACQ <1.5) compared to subjects with uncontrolled asthma (ACQ $\geq 1.5)$ [6.1 (5.4-7.3) versus 5.6 (4.9-6.4), respectively, $p=0.014]$. This study demonstrates that controlled asthma is associated with a higher O3I, suggesting that LCn-3PUFA may have a role in asthma management. However, intervention studies are needed to confirm our observations and to investigate the optimal O3I needed to elicit improvements in asthma outcomes.

\subsection{Anticancer Properties of Krill Oil Are Associated with Intrinsic Apoptotic Pathway}

A.G. Jayathilake ${ }^{1}$, K. Nurgali ${ }^{1}$, R. Luwor ${ }^{2}$ and X.Q. Su ${ }^{1}$

${ }^{1}$ Victoria University, Melbourne, Melbourne, VIC, Australia

2 Department of Surgery, University of Melbourne, Melbourne, VIC, Australia

The currently available chemotherapy for colorectal cancer (CRC) associates with side effects. The effective nutraceuticals with high anticancer efficacy and low side effects are desirable. The aim of this study was to investigate the anticancer properties of krill oil (KO) - a rich source of eicosapentaenoic acid (EPA) and docosahexaenoic acid (DHA) - on human CRC cells.

Human CRC cell lines DLD-1, HT-29 and LIM-2405 were treated with the free fatty acid extract (FFAE) of KO, EPA and DHA for $24 \mathrm{~h}$ and $48 \mathrm{~h}$. The effects of treatments on cell proliferation, mitochondrial membrane potential and reactive oxygen species (ROS) were determined using commercial kits. The expression of caspase- 9 and caspase- 3 following treatments was determined via western blotting and immunohistochemistry. The FFAE of KO, EPA and DHA significantly inhibited the cell proliferation in all three cell lines and reduced the formation of ROS. Treatments with the FFAE of KO, EPA and DHA (2:1) resulted in a significant increase in the mitochondrial membrane potential $(p<0.01)$. Furthermore, the expression of caspase- 9 and caspase- 3 increased significantly in all cell lines following the FFAE treatment.

The results of this study provide evidence of the antiproliferative effects of $\mathrm{KO}$ associated with the activation of the intrinsic (mitochondrial) apoptotic pathway.

\subsection{The Impact of Dietary Fibre Intake on Markers of Inflammation in Adolescents}

\section{O.G. Swann ${ }^{1}$, M. Kilpatrick ${ }^{1}$, M. Breslin ${ }^{1}$, T. O'Sullivan ${ }^{2}$, L. Beilin ${ }^{3}$, T. Mori ${ }^{3}$ and W.H. Oddy $^{1}$}

${ }^{1}$ Menzies Institute for Medical Research, University of Tasmania, Hobart, TAS, Australia

2 Edith Cowan University, Perth, WA, Australia

${ }^{3}$ University of Western Australia, Perth, WA, Australia

An increased intake of dietary fibre may be beneficial for reducing chronic inflammation, with reduction in inflammatory markers and improvements in inflammatory conditions associated with 
high dietary fibre intake. We aimed to examine the relationship between dietary fibre intake and the inflammatory markers high-sensitivity C-reactive protein (hs-CRP) and leptin, and anti-inflammatory adiponectin in 17 year-olds from the Western Australian Pregnancy Cohort (Raine) Study ( $n=677$ ) using tobit and linear regression. After excluding those with hs-CRP $>10 \mathrm{mg} / \mathrm{L}$, higher dietary fibre intake was associated with lower log hs-CRP concentrations $(\beta=-0.01), \log$ leptin $(\beta=-0.02)$ and adiponectin $(\beta=-0.04)$ before adjustment for energy intake. Female sex and oral contraceptive usage were associated with higher hs-CRP, leptin and adiponectin. Increased BMI was associated with higher hs-CRP and leptin and lower adiponectin. Lower hs-CRP, leptin and adiponectin were observed with increasing energy intake. Higher physical activity was associated with decreases in hs-CRP, leptin, and adiponectin. Associations between dietary fibre and hs-CRP, leptin and adiponectin were attenuated by adjustment for energy intake, suggesting that alternate factors associated with energy intake contribute to the association. Our results indicate that physical activity may play a greater role than diet, with the largest correlations with hs-CRP, leptin and adiponectin.

\subsection{Agreement between Markers of Population Iodine Status: A Systematic Review}

M. Wassie ${ }^{1}$, P. Middleton ${ }^{2,3}$ and S.J. Zhou ${ }^{1,2}$

${ }^{1}$ School of Agriculture, Food and Wine, The University of Adelaide, Adelaide, SA, Australia

${ }^{2}$ Robinson Research Institute, School of Paediatrics and Reproductive Health, The University of Adelaide, Adelaide, SA, Australia

3 South Australian Health and Medical Research Institute, The University of Adelaide, Adelaide, SA, Australia

Population iodine deficiency is indicated by $>3 \%$ of the population with neonatal thyroid stimulating hormone (TSH) concentration $>5 \mathrm{mIU} / \mathrm{L}$, median urinary iodine concentration (MUIC) $<100 \mu \mathrm{g} / \mathrm{L}$ or the prevalence of goitre $>5 \%$ in school age children. However, the agreement between these markers has not been systematically investigated. We performed a systematic search for studies published on PubMed, Scopus, CINAHL, Embase and PsycINFO. Studies assessing iodine status in the population using TSH marker and either MUIC or goitre prevalence in school age children were included. Of 15,619 records identified, 56 eligible studies were included in the review. Agreement between markers when classifying the iodine status of populations into the same category was $64 \%$ for studies which classified iodine status based on TSH and MUIC, and $83 \%$ for studies which classified iodine status based on TSH and goitre prevalence. The TSH marker had a sensitivity of 0.75 and specificity of 0.50 compared with MUIC, and 0.86 and 0.50 compared with goitre prevalence. The TSH marker had a better agreement with goitre prevalence than MUIC in classifying the iodine status of populations. Reevaluation of the criteria based on the different markers used to classify iodine status of populations is warranted.

\subsection{Association between Dietary Variety and Depressive Symptoms in Urban Dwelling Elderly}

K. Yamamoto ${ }^{1}$, K. Motokawa ${ }^{2}$, Y. Takahiro ${ }^{1}$, Y. Tomohiro ${ }^{1}$ and W. Yutaka ${ }^{2}$

${ }^{1}$ Graduate School of Food and Nutritional Science, Toyo University, Ora-gun, Gunma, Japan

2 Tokyo Metropolitan Institute of Gerontology, Itabashi-ku, Tokyo, Japan

Depressive symptoms in the elderly constitute a serious problem requiring novel methods for improvement. Importantly, depressive symptoms have been associated with dietary food intake and dietary pattern in adolescence. Thus, in this study we examined the relationship between dietary variety and depressive symptoms, in order to determine the feasibility of screening for elderly people with a tendency to develop depressive symptoms by using a simple evaluation of their eating habits. A survey was conducted among urban dwelling elderly $(n=1326)$. This was a cross-sectional study. Depressive symptoms and dietary variety were assessed by using the General Depression Scale and the dietary variety score (DVS; a 10-item food frequency questionnaire), respectively. The relationship 
between tertile category of the DVS and depressive symptoms was examined by using multivariable logistic regression. In the fully adjusted model, the odds ratios for depressive symptoms were 0.87 (95\% confidence interval, $0.62-1.22)$ and 0.60 (95\% confidence interval, $0.39-0.91)$, respectively, for participants in the category of DVS, compared with those in the lowest category ( $p$ for trend $=0.004$ ). Thus, habitual consumption of a well-balanced diet may be associated with a lower prevalence of depressive symptoms in urban dwelling elderly.

2.89. Effects of Acute Changes in Fermentable Fibre Intake on Regional Colonic Fermentation and Transit in Patients with Quiescent Ulcerative Colitis

C.K. Yao, R. Burgell, K. Taylor, M. Ward, A. Friedman, J. Barrett, J. Muir and P. Gibson

Monash University, Prahran, VIC, Australia

Reduced colonic fermentation has been described in patients with quiescent ulcerative colitis (UC). Acute variations in fibre intake may impact such defects but is poorly studied. Hence we aimed to define colonic fermentation in quiescent UC patients following acute variations in fibre intake. In a randomised, cross-over trial, 15 quiescent UC patients and nine controls were recruited. After a 7-day run-in, subjects were fed two meals high $(13 \mathrm{~g})$ or low $(<1 \mathrm{~g})$ in fermentable fibre before ingesting a wireless pH-motility capsule. Subjects then crossed over to the other diet three days after passage of the capsule. End points were diet-associated differences in colonic transit time (CTT) and regional pH. Overall (mean 6.3(95\% CI: 6.0-6.5) vs. 6.9(6.5-7.2); $p=0.02$ ) and distal colonic $\mathrm{pH}(8.2(8.0-8.5)$ vs. 7.7(7.4-8.0); $p=0.04$ ) were decreased in controls with high vs. low fibre diets. In UC patients, only caecal $\mathrm{pH}$ was decreased after high fibre intake (5.2(5.0-5.4) vs. low 5.6(5.3-5.7); $p=0.001$ ). No differences in CTT were observed between diets in either cohorts but subgroup analysis in UC patients showed highly heterogeneous responses to a high fibre diet. In conclusion, contrary to controls, abnormalities in motility and the functional capability of the colonic microbiota may exist in patients with quiescent UC.

2.90. Is Dairy Consumption Associated with Depressive and Anxiety Symptoms in Adults? A Systematic Literature Review of Observational Studies

M. Hockey, A. McGuinness, W. Marx, T. Rocks, F.N. Jacka and A. Ruusunen

Food and Mood Centre, IMPACT SRC, School of Medicine, Deakin University, Geelong, VIC, Australia

Diet is an important modifiable risk factor for depression. However, the association between dairy consumption and depression is unclear. This systematic review aimed to synthesise evidence on the association between dairy consumption and depressive and anxiety symptoms. A systematic search of CINAHL, Cochrane, MEDLINE complete, EMBASE, Scopus and PsycINFO databases was conducted from database inception to February 2018. Case-control, cohort or cross-sectional studies that reported on the association between dairy consumption (total dairy, milk, yoghurt or cheese) and depression or anxiety in adults were included. Thirteen studies ( $n=7$ cohort, $n=6$ cross-sectional), including a total of 58,220 participants, were identified; $38 \%$ reported no association. Statistically significant inverse associations were reported $(n=4)$ between intakes of low-fat dairy, milk, yoghurt and whole-fat yoghurt and depression. Conversely, positive associations were reported $(n=5)$ between intakes of total dairy, milk, yoghurt, low-fat yoghurt and cheese and depression. Gender was identified as a potential effect modifier of this association. No studies reported on dairy consumption and anxiety. This is the first review to examine the association between dairy consumption and depressive and anxiety symptoms. Evidence is suggestive of an association, although inconsistent findings make firm conclusions difficult. Future interventions are required to ascertain directions of these associations. 


\subsection{Dietary Patterns and Associations with Psychological Distress-A Study of Undergraduate Nurses}

S. Khalesi and S. Williams

Physical Activity Research Group, Appleton Institute and School of Health, Medical and Applied Sciences, Central Queensland University, Rockhampton, QLD, Australia

Undergraduate nursing studies are physically and mentally demanding, and the nature of their clinical activities can impose a high level of psychological distress that impairs academic performance and wellbeing. Many undergraduate students adopt unhealthy eating intakes and behaviours and for nurses, this may further impair cognitive function, mental health and increase psychological distress. This cross-sectional study explored associations between dietary patterns and psychological distress in a cohort of nursing students $(n=321)$. Dietary patterns were derived from a food frequency questionnaire using explanatory factor analysis. Psychological distress was assessed using the Depression Anxiety Stress Scale. Associations were analysed using linear regression controlled for sociodemographic variables. Compared to the Australian Dietary Guidelines, students' intakes of fruit $(1.7 \pm 2.0)$ and vegetables $(3.2 \pm 2.7)$ were lower and discretionary foods $(3.7 \pm 3.4)$ were higher. Three dietary patterns were extracted: 'Western', 'Australian' and 'Healthy'. 'Western' dietary pattern (loaded with processed foods, fat, sugar and salt) was associated with increased depression (OR: 5.8, $95 \%$ CI: $1.2,10.4$ ) and stress (OR: 4.7, 95\% CI: $0.8,9.4$ ) scores. Overall, poor dietary habits of nursing students were linked to increased risk of psychological distress. Interventions to improve dietary intakes of nursing students may improve their mental health, wellbeing and academic performance.

2.92. The Effect of Saffron Supplementation on Symptoms of Depression and Anxiety: A Systematic Review and Meta-Analysis

W. Marx ${ }^{1}$, M. Lane ${ }^{1}$, T. Rocks ${ }^{1}$, A. Ruusunen ${ }^{1}$, A. Loughman ${ }^{1}$, A. Lopresti ${ }^{2}$, S. Marshall ${ }^{3}$, M. Berk ${ }^{4,5}$, F. Jacka ${ }^{1,6,7}$ and O. Dean ${ }^{4,5,8}$

${ }^{1}$ Food \& Mood Centre, Deakin University, Geelong, VIC, Australia

${ }^{2}$ School of Psychology and Exercise Science, Murdoch University, Perth, WA, Australia

${ }^{3}$ Faculty of Health Sciences \& Medicine, Bond University, Robina, QLD, Australia

${ }^{4}$ IMPACT SRC, Deakin University, Geelong, VIC, Australia

${ }^{5}$ Department of Psychiatry, University of Melbourne, Melbourne, VIC, Australia

${ }^{6}$ Centre for Adolescent Health, Murdoch Children's Research Institute, Melbourne, VIC, Australia

7 Black Dog Institute, Sydney, NSW, Australia

8 Florey Institute for Neuroscience and Mental Health, University of Melbourne, Melbourne, VIC, Australia

Saffron (Crocus sativus L.) has gained interest as a potential treatment in psychiatry. The aim of this systematic review and meta-analysis was to explore the effect of saffron supplementation on symptoms of depression and anxiety. Using the PRISMA guidelines, randomised controlled trials that investigated the use of saffron supplementation for depression and anxiety were identified using a systematic search of relevant databases. Where sufficient outcome data was available, data were pooled using meta-analysis. Twenty-three studies $(N=1237$ participants) were included. Saffron had a large positive effect size when compared to placebo for depressive symptoms ( $\mathrm{g}=0.99,95 \% \mathrm{CI}$ : 0.61 , $1.37, p<0.001)$ and anxiety symptoms ( $g=0.95,95 \%$ CI: $0.27,1.63, p<0.001)$. Saffron also had a large positive effect size when used as an adjunct to antidepressants for depressive symptoms ( $\mathrm{g}=1.23$, $95 \%$ CI: $0.13,2.33, p=0.028$ ). Compared to antidepressants, there was no significant difference between groups for depressive symptoms ( $\mathrm{g}=-0.17,95 \% \mathrm{CI}$ : 0.-0.50, $0.17, p=0.33$ ). Egger's regression test found evidence of publication bias. Preliminary evidence suggests saffron could be an effective and well-tolerated intervention for symptoms of depression and anxiety. However, due to evidence of publication bias, lack of regional diversity and methodological limitations of existing studies, further trials are required. 
2.93. Effects of A Highly Bioavailable Curcumin Extract on Mood and Memory in Healthy Older Adults: A 12 Week Double-Blind, Placebo-Controlled Trial

\section{A. Scholey, K.H.M. Cox, A. Pipingas and D. White}

Swinburne University, Hawthorn, VIC, Australia

We have previously reported that 4-week administration of a highly bioavailable curcumin extract (Longvida ${ }^{\mathrm{TM}}$ ) improved working memory and reduced fatigue and stress reactivity in older Australians. This follow-up study (ACTRN12616000484448) aimed to determine if similar effects were evident following 12 weeks administration of the same extract. In this double-blind, placebo-controlled, parallel-groups trial, 80 participants aged 50-80 years (mean $=68.1$, SD 6.34) were randomised to receive daily administration of Longvida ${ }^{\mathrm{TM}}$ (400 mg containing $80 \mathrm{mg}$ curcumin) or placebo. Assessment at baseline then at 4 and 12 weeks included measures of cognitive performance, mood, neuroimaging, cardiovascular function and other blood biomarkers. Compared with the placebo group, at 4 weeks the curcumin group had significantly lower scores on the Profile of Mood States (POMS) dimensions of fatigue at both 4 and 12 weeks, and of tension, anger, confusion and total mood disturbance at 4 weeks only. The curcumin group also had significantly better working memory performance at 12 weeks, as measured by Serial Threes, Serial Sevens and virtual Water Maze scores. Curcumin was also associated with better performance on a pattern separation task. These results confirm that Longvida $^{\mathrm{TM}}$ improves aspects of mood and working memory in a healthy older population.

\subsection{Curcumin and Long-Chain Omega-3 Polyunsaturated Fatty Acids Supplementation Reduces} Insulin-Related Markers of Alzheimer's Disease Risk: A Randomised Controlled Study

R.N. Thota ${ }^{1}$, J. Rosato ${ }^{1}$, T. Burrows ${ }^{1}$, C. Dias $^{2}$, R. Martins ${ }^{2}$ and M. Garg $^{1}$

${ }^{1}$ University of Newcastle, Callaghan, NSW, Australia

2 Macquarie University, Sydney, NSW, Australia

A growing body of molecular evidence suggests that type 2 diabetes and Alzheimer's disease (AD) share common pathophysiology, particularly pathways involving insulin resistance. This study investigated the effects of curcumin and long-chain omega-3 polyunsaturated fatty acids (LCn-3PUFA) supplementation on insulin-related risk markers (glycogen synthase kinase-3 $\beta$, GSK-3 $\beta$ and islet amyloid polypeptide, IAPP) of AD in individuals with high risk of type 2 diabetes. A $2 \times 2$ factorial, double-blinddouble-blinded, randomised, placebo-controlled trial was performed. Participants received either a placebo or curcumin or fish oil or curcumin plus fish oil for 12 weeks. Plasma levels of GSK-3 $\beta$ and IAPP were determined using ELISA assays. Postintervention, plasma levels of GSK-3 $\beta(-2.4 \pm 0.4 \mathrm{ng} / \mathrm{mL})$ IAPP $(-2.0 \pm 0.8 \mathrm{ng} / \mathrm{mL})$ and HOMA2-IR $(-0.3 \pm 0.08)$ were reduced in the CC group $(p<0.05)$. FO supplementation resulted in a similar significant reduction in GSK-3 $\beta$ $(-2.6 \pm 0.5 \mathrm{ng} / \mathrm{mL})$ and IAPP $(-2.4 \pm 0.8 \mathrm{ng} / \mathrm{mL})$ when compared with PL group $(p<0.05)$. The combined $(\mathrm{CC}+\mathrm{FO})$ treatment resulted in a higher reduction $(-3.2 \pm 0.8 \mathrm{ng} / \mathrm{mL})$ in IAPP; however, the reduction in GSK-3 $\beta(2.5 \pm 0.7 \mathrm{ng} / \mathrm{mL})$ was similar to CC and FO treatments. Dietary supplementation with curcumin and/or LCn-3PUFA reduces IAPP and GSK-3 $\beta$, suggesting a potential role for these bioactive compounds in the prevention of AD in high risk individuals.

\subsection{An In Vivo Magnetic Resonance Spectroscopic Study: Effect of Sialylated Milk Oligosaccharides Intervention on Brain Metabolites in Piglets}

B. Wang ${ }^{1}$, H.X. Wang ${ }^{1}$, Y. Chen ${ }^{1}$, Z. Haque ${ }^{1}$, M. de Veer ${ }^{2}$ and G. Egan ${ }^{2}$

${ }^{1}$ Charles Sturt University, Wagga Wagga, NSW, Australia

2 Monash Biomedical Imaging, Monash University, Clayton, VIC, Australia

Human milk contains high concentrations and diversity of sialylated oligosaccharides that have multifunctional health benefits; however their potential role in optimising neurodevelopment by 
modulating brain metabolites and neurotransmitters remains unknown. Piglets were randomly allocated to one of three groups and fed either standard sow milk replacer (SMR) alone $(n=15)$, SMR supplemented with sialyllactose (SL, $n=16$ ) or a combination of SL and 6 '-sialyllactosamine (SL/SLA, $n=15$ ) for 35 days. Brain spectra were acquired using a 3T MRS system at short and long echo times ( $\mathrm{TE}=35 \mathrm{~ms}$ and $270 \mathrm{~ms}$ ). Sialylated milk oligosaccharides (SMOs) fed piglets were observed to have significantly increased the absolute levels of myo-inositol (Ins) and glutamate + glutamine (Glx), in particular, the SL group. Similar findings were found in the relative amount of these metabolites calculated as ratios to creatine $(\mathrm{Cr})$, choline (Cho) and $\mathrm{N}$-acetylaspartate (NAA), respectively $(p<0.05)$. There were significant positive correlations of frontal lobe Ins, glutathione (Glth), NAA, total NAA (TNAA), total Cho (TCho), scyllo-Inositol (SI) and total Cr (TCr) with total white matter volume $(p<0.01)$ at TE270. Milk SMOs can alter many important brain metabolites and neurotransmitters required for optimising neurodevelopment in piglets, an animal model of human infants.

\subsection{Effects of Cross-Sex Hormone Treatment on Body Composition, Metabolic Parameters and} Docosahexaenoic Acid Levels in Transgender Individuals

K. Abbott ${ }^{1,2}$, K-J. Wynne ${ }^{3,4,5}$, J. Luu ${ }^{3}$, L. Wood ${ }^{2,5}$ and M. Garg ${ }^{1,2}$

${ }^{1}$ Nutraceuticals Research Program, University of Newcastle, Callaghan, NSW, Australia

${ }^{2}$ School of Biomedical Sciences \& Pharmacy, University of Newcastle, Callaghan, NSW, Australia

${ }^{3}$ Department of Endocrinology, John Hunter Hospital, Newcastle, NSW, Australia

${ }^{4}$ School of Public Health and Medicine, University of Newcastle, Callaghan, NSW, Australia

${ }^{5}$ Hunter Medical Research Institute, Newcastle, NSW, Australia

Cross-sex hormone (CSH) treatment facilitates gender transition in transgender individuals. Sex hormones influence type 2 diabetes (T2D) and cardiovascular disease (CVD) risk and modulate body composition and endogenous docosahexaenoic acid (DHA) metabolism. The aim of this pilot study is to examine the effects of CSH treatment on body composition and cardiometabolic parameters across the first six months of CSH treatment. In total, $n=23$ (M2F: $n=11 ;$ F2M: $n=12$ ) participants (age: $24.9 \pm 9.4$ years; BMI: $27.4 \pm 7.6 \mathrm{~kg} / \mathrm{m}^{2}$ ) were recruited from the endocrine clinic at John Hunter Hospital (Newcastle, Australia). Measurements were recorded at baseline, three and six months. Body composition was measured by DEXA and fatty acid composition of erythrocytes by gas chromatography. M2F treatment decreased skeletal muscle mass (SMM) at three $(-0.94 \pm 1.1 \mathrm{~kg}, p=0.041)$ and six $(-3.05 \pm 1.8 \mathrm{~kg}, p=0.047)$ months, and increased body fat mass $(+2.75 \pm 0.6 \mathrm{~kg}, p=0.003)$ at six months. F2M increased SMM $(+2.7 \mathrm{~kg} \pm 2.1 \mathrm{~kg}, p=0.025)$ at three and six $(+4.20 \pm 1.9 \mathrm{~kg}, p=0.003)$ months. Testosterone reduced and oestrogen increased erythrocyte DHA after three months. CSH treatment alters CVD risk parameters (body composition, metabolic profile and erythrocyte DHA). Longer term follow-up studies, with a larger sample size are warranted to determine long-term impact of CSH treatment on T2D and CVD risk.

\subsection{In Vitro Investigation of Sweet Cherry Extract}

M.L. Blackhall ${ }^{1}$, R. Berry ${ }^{1}$, K. Ahuja ${ }^{2}$, D.P. Geraghty ${ }^{2}$ and J.T. Walls ${ }^{1}$

${ }^{1}$ College of Health and Medicine, University of Tasmania, Hobart, TAS, Australia

${ }^{2}$ College of Health and Medicine, University of Tasmania, Launceston, TAS, Australia

Inflammation is acknowledged as a precursor to many diseases, with water-soluble flavonoids (e.g., anthocyanins) implicated in reducing this disease risk. The aim of the present study was to determine whether sweet cherry anthocyanins (SCA) can reduce the release of inflammatory mediators from lipopolysaccharide (LPS)-stimulated RAW267.4 macrophages. The cells were first treated with SCA to understand the impact of concentration on cell toxicity and markers of inflammation. Concentrations of the inflammatory mediators IL-6, IL-10 and GM-CSF were observed. Following treatment with 10,50 and $100 \mathrm{mM} \mathrm{SCA}$, the viability of the cells were $113 \pm 0.65,113 \pm 1.31$ 
and $99 \pm 0.63 \%$, respectively, as compared to the untreated control (100 $\pm 0.24 \%)$. These results indicate that up to $100 \mathrm{mM} \mathrm{SCA}$ did not adversely affect cell growth. In addition when treated with SCA, the secretion of inflammatory mediators was significantly less in the LPS-stimulated cells. IL-10 concentration of treated cells was $69.6 \%$ lower than untreated cells $(p=0.015)$, whereas GM-CSF concentration was $46 \%$ lower in treated cells $(p=0.0007)$. Observations from the in vitro studies suggest that the SCA are inhibiting production of GM-CSF from the macrophages at a cellular level, which has implications for understanding the mechanism of attenuating inflammation.

\subsection{Influence of Exercise Management on Fasting Plasma Homocysteine Concentration in Female Long-Distance Runners}

A. Shinagawa, A. Minematsu, T. Yamazaki, R. Furuki, Y. Miyakoshi, T. Yano and M. Ota

TOYO university, Izumino Itakura Cho, Oura-gun, Japan

Recently, plasma homocysteine (Hcy) concentration has attracted attention as an indicator of bone quality. Plasma Hcy concentration is reported to change with diet and exercise; however, previous studies of athletes have lacked consistency. This study was under taken to investigate differences in plasma Hcy during exercise management under specific dietary conditions.

Subjects in this study comprised six college female long-distance runners. During the exercise management period, running exercise was restricted, whereas in the nonmanagement period, running was performed in the usual manner. Dietary energy and methionine levels were set to $2100 \pm 100 \mathrm{kcal}$ and $\leqq 2000 \mathrm{mg}$, respectively, for both periods. Fasting blood samples were collected from all subjects. For statistical analysis, the Wilcoxon Signed-rank test was performed with SPSS (ver. 24) $(p<0.05)$. Hcy concentrations in plasma during the management and nonmanagement periods were 4.37 and $7.28 \mathrm{nmol} / \mathrm{mL}$, respectively. Dietary conditions were kept constant; the plasma Hcy concentration was significantly lower when exercise was regulated than when it was not. Because daily diet and exercise intensity in athletes differ from those in typical adults, it is necessary to adjust diet and exercise conditions in order to measure plasma Hcy concentrations in athletes.

2.99. Pure Omega-3 Polyunsaturated Fatty Acids (n-3FA) Are Associated with Increased Plasma Levels of 3-Carboxy-4-methyl-5-propyl-2-furanpropanoic Acid (CMPF) in a Short-Term Study in Women

L. Ge ${ }^{1}$, R.A. Gibson ${ }^{1}$, D. $\mathrm{Li}^{2}$, X.-F. Guo ${ }^{2}$ and A.J. Sinclair ${ }^{3,4}$

${ }^{1}$ Wine and Food Science, University of Adelaide, Adelaide, SA, Australia

${ }^{2}$ Food Science and Nutrition, Zhejiang University, Hangzhou, China

${ }^{3}$ Deakin University, Waurn Ponds, VIC, Australia

${ }^{4}$ Department of Nutrition, Dietetics and Food, Monash University, Nottinghill, VIC, Australia

CMPF is a metabolite of furan fatty acids (FurFA) found in plasma and excreted in urine. FurFA are widespread in nature, including fish in particular. Diets rich in fish oil and fish have been associated with significant elevations in plasma levels of CMPF; therefore, CMPF may be an excellent plasma biomarker of fish/fish oil intake. We measured the plasma CMPF levels in 10 healthy women who consumed $1 \mathrm{~g}$ daily of pure eicosapentaenoic acid (EPA), docosapentaenoic acid (DPA) or docosahexaenoic acid (DHA), or olive oil for six days, in a cross-over study. Plasma levels of the n-3FA and CMPF were measured after collection on blood dried technology cards followed by GC-FID and GCLC-MS/MS. After six days, there were significant increases in plasma levels of EPA, DPA and DHA $(p<0.05)$. No significant changes in n-3FA were recorded in the olive oil treatment phase. Plasma CMPF levels were significantly increased after just six days of treatment with the n-3FA, but not with olive oil: fold increases (day 6/day 0 ) were $0.82 \times$ in the olive oil treatment, compared with $1.77 \times$ for EPA, $2.53 \times$ for DPA and $1.62 \times$ for DHA. The metabolic pathway from n-3FA to CMPF remains to be elucidated. 
2.100. TAS2R4 Variants May Predict Adenomatous Colorectal Polyp Risk

A. Turner ${ }^{1}$, M. Lucock ${ }^{2}$, M. Veysey ${ }^{3}$, S. Keely ${ }^{4,5}$ and E. Beckett ${ }^{1,5}$

${ }^{1}$ School of Medicine and Public Health, University of Newcastle, Newcastle, NSW, Australia

${ }^{2}$ School Environmental and Life Sciences, University of Newcastle, Newcastle, NSW, Australia

${ }^{3}$ Hull-York Medical School, University of York, Heslington, York, UK

${ }^{4}$ School of Biomedical Sciences and Pharmacy, University of Newcastle, Newcastle, NSW, Australia

${ }^{5}$ Hunter Medical Research Institute, Newcastle, NSW, Australia

Adenomatous polyps (AP) are the benign precursor to the majority of colorectal cancers. As the second most commonly diagnosed cancer in Australia, a better understanding of the risk factors and development of this disease is vital. Diet-related risk factors have been identified; however, these do not fully explain risk. The TAS2R family of receptors, expressed throughout the gastrointestinal tract, respond to bitter compounds. TAS2Rs may be involved in modulating risk for AP and CRC via their impact on taste preferences and diet, or via modulation of gastrointestinal functions. This study aimed to assess the role of polymorphisms in TAS2R4 in risk for AP. DNA was extracted from blood (QiAMP $^{\mathrm{TM}}$ DNA Mini Kit; $n=168 ; 25 \%$ AP) of routine colonoscopy patients, and genotyping was carried out (Taqman assays). Two variants of the TAS2R4 gene were assessed for association with AP (rs2234001 and rs2233998). It was found that presence of the rs2234001 G allele was related to increased risk for $\mathrm{AP}$ (odds ratio $=5.7 ; 95 \% \mathrm{CI}=1.3-25.5 ; p=0.02$ ). Overall, this study suggests that TAS2R4 polymorphisms may be related to risk for AP, however further investigations are needed to confirm the mechanisms and a causative link between TAS2R genotypes and risk for AP.

\subsection{Sociodemographic Characteristics Associated with Family Meal Frequency in Young Children}

\section{E.T. Pitson, A. Spence and R. Laws}

Deakin University, Burwood, VIC, Australia

Australian children's diets do not meet dietary guidelines, leading to high rates of overweight and obesity. Family meals have been associated with healthier diets and may be a useful target for nutrition promotion, however little is known about what influences family meal frequency in young children. Parents of children aged six months to six years completed an online survey in $2014(n=992)$. Parents reported sociodemographic characteristics and the weekly frequency of the whole family eating dinner together. Variables which showed significant associations in univariate regression analyses $(p<0.05)$, were included in multiple ordered logistic regression. Preliminary analysis using that model showed family dinner frequency was positively associated with child age (OR 1.13 95\% CI: 1.02, 1.25) and having siblings (OR $1.6895 \%$ CI: 1.25, 2.27), and negatively associated with paternal education (OR 0.70 95\% CI: 0.53, 0.91) and living in more affluent areas (OR 0.82 95\% 0.68-0.98). Characteristics not associated with family dinner frequency were parent country of birth, maternal education and maternal and paternal employment status. In contrast to expectations, characteristics reflecting higher socioeconomic position were either not associated or inversely associated with family dinner frequency, highlighting need for further investigation into mechanisms linking socioeconomic position and family meals and the ultimate influence on child diets.

\subsection{Preworkout Supplement Use and Caffeine Intake among Active Australians}

K. Barnes ${ }^{1}$, H. O'Conner ${ }^{2}$, G. Slater ${ }^{3}$, G. Grant ${ }^{4}$, S. Hall ${ }^{4}$ and B. Desbrow ${ }^{1}$

${ }^{1}$ School of Allied Health Sciences, Griffith University, Southport, QLD, Australia

2 Faculty of Health Sciences and Charles Perkins Centre, University of Sydney, Camperdown, NSW, Australia

${ }^{3}$ School of Health and Sport Sciences, University of the Sunshine Coast, Sippy Downs, QLD, Australia

${ }^{4}$ School of Pharmacy \& Menzies Health Institute, Griffith University, Southport, QLD, Australia 
Preworkout supplements (PWS) are multi-ingredient dietary supplements claiming ergogenic effects. Caffeine is a common ingredient of PWS and is present at variable doses. While generally regarded as safe, caffeine can cause severe adverse events in doses $>400 \mathrm{mg} /$ day. This study describes active individual's awareness, beliefs and use of PWS in Australia. A 10-min online survey identified PWS practices, beliefs and knowledge of caffeine sources. Snowball and convenience sampling recruited participants at Fitness Expo 2017, and via mass email to a University population. Of 377 respondents, $66 \%(n=248)$ currently or previously used a PWS, with $27 \%(n=66)$ of users consuming a PWS twice daily. Users were most likely to be male, and to report 'training regularly'. Users more frequently reported positive perceptions of PWS for strength and concentration in comparison to nonusers. Participants were unable to accurately quantify caffeine in common dietary sources or in PWS, and more than one quarter of users was estimated to consume caffeine above the recommended $400 \mathrm{mg} /$ day. Adverse side effects from PWS were reported by $27 \%(n=66)$ of users including elevated heart rate, tremors and increased anxiety. Active individuals were unable to estimate caffeine intake accurately and are at risk of adverse caffeine related effects when using PWS.

\subsection{Hydrogen-Rich Water and Its Effect on Lactate Removal and Oxygen Utilisation during Incremental Exercise}

M. Botek, B. Sládečková, J. Krejčí and A. McKune

Department of Natural Sciences in Kinanthropology, Palacky University, Olomouc, Czech Republic

Hydrogen-rich water (HRW) ingestion has potential health and exercise performance benefits due to its antioxidant, anti-inflammatory and alkaline properties. This randomised, double-blind, placebo-controlled, cross-over study investigated the effects of HRW ingestion on physiological, metabolic and perceptual responses to cycling. Ten males (age $=26.4 \pm 3.1$ years, $\mathrm{BMI}=23.1 \pm 1.9 \mathrm{~kg} \cdot \mathrm{m}^{-2}, \%$ body fat $=11.3 \pm 3.0 \%, \mathrm{VO}_{2} \max =54.5 \pm 4.7 \mathrm{~mL} \cdot \mathrm{kg}^{-1} \cdot \mathrm{min}^{-1}$ ) ingested $600 \mathrm{~mL}$ ( $300 \mathrm{~mL}$ at $30 \mathrm{~min}$ pre, $300 \mathrm{~mL}$ at $1 \mathrm{~min}$ pre) of either $\mathrm{HRW}$ or placebo before performance of an incremental cycle ergometer test $\left(10 \mathrm{~min}\right.$ at $1 \mathrm{~W} \cdot \mathrm{kg}^{-1}, 8 \mathrm{~min}$ at $2 \mathrm{~W} \cdot \mathrm{kg}^{-1}, 8 \mathrm{~min}$ at $3 \mathrm{~W} \cdot \mathrm{kg}^{-1}$ and $8 \mathrm{~min}$ at $4 \mathrm{~W} \cdot \mathrm{kg}^{-1}$ ). Physiological, metabolic and perceptual responses were compared in the last minute of each stage. Ingestion of HRW resulted in significantly lower lactate $(9.0 \pm 2.4$ vs. $10.3 \pm 3.1 \mathrm{mmol} / \mathrm{L}$; $p<0.05)$ production and ventilatory equivalent for oxygen $\left(\mathrm{VE} / \mathrm{VO}_{2}\right)$ ratio $(32.4 \pm 7.9$ vs. $35.0 \pm 8.6$; $p<0.05)$ compared with placebo during high intensity $\left(4 \mathrm{~W} . \mathrm{kg}^{-1}\right)$ exercise. Supplementation with $600 \mathrm{~mL}$ of HRW during the $30 \mathrm{~min}$ prior to exercise may enhance high intensity performance through increased lactate utilisation and oxygen extraction efficiency.

\subsection{Athletic Retirement: The Unexplored Effect of Sporting Culture on An Individual's Relationship with Food and Body}

G. Buckley ${ }^{1}$, L. Hall ${ }^{2}$, R. Belski ${ }^{1}$ and A. Lassemillante ${ }^{1}$

${ }^{1}$ Faculty of Health, Arts \& Design, Swinburne University of Technology, Hawthorn, VIC, Australia

${ }^{2}$ Consumer Representative, Melbourne, VIC, Australia

Retirement from elite sport is a unique transition that influences significant lifestyle changes. This paper reviews the literature surrounding the effect of athletic retirement on maladaptive eating behaviours and body dissatisfaction. A systematic literature review was conducted using the PRISMA statement using the search terms retir* or former AND athlete* or sport AND eat* or feed* or food or disorder* or anorexi* or bulimi* or weight or 'body image.' The following databases were searched; Web of Science, Scopus, PubMed, EBSCO Host, Sport Discus and CINAHL for thematic and quantitative analysis. Eighteen studies were included in the synthesis where three themes emerged: (1) influence of sporting culture; (2) body dissatisfaction and body ideal transition; and (3) disordered eating and energy compensation. The synthesis explores how maladaptive behaviours can arise through sustained athletic identity, lack of awareness or social support, contradictory body ideals and 
compensatory behaviours. There is currently insufficient evidence about the prevalence of disordered eating, compulsive exercise and other maladaptive compensatory behaviours to adequately support athletes into retirement. Furthermore, it is the responsibility of sporting organisations to continue to support athletes into retirement to prevent the development of potentially lifelong and maladapted behaviours relating to disordered eating and body image.

\subsection{Caffeine Content of Preworkout Supplements Commonly Used by Australian Consumers}

B. Desbrow ${ }^{1}$, S. Hall ${ }^{2}$, H. O'Connor ${ }^{3}$, G. Slater ${ }^{4}$, K. Barnes ${ }^{1}$ and G. Grant ${ }^{2}$

${ }^{1}$ School of Allied Health Sciences, Griffith University, Gold Coast, QLD, Australia

${ }^{2}$ School of Pharmacy and Pharmacology, Griffith University, Gold Coast, QLD, Australia

3 Faculty of Health Sciences and Charles Perkins Centre, The University of Sydney, Sydney, NSW, Australia

${ }^{4}$ School of Health and Sport Sciences, University of the Sunshine Coast, Sippy Downs, QLD, Australia

The stimulant properties of caffeine are often promoted in 'preworkout' supplements (PWS) to assist with training, reduce the perception of fatigue and, for some brands, assist body fat loss. While manufacturers of PWS often indicate the inclusion of significant amounts of caffeine, no independent verification of the caffeine content of these products exists. The aim of this investigation was to independently assess the caffeine content of popular PWS in Australia and compare these values to nutrition information panel data. Fifteen PWS were tested for their caffeine content (both within and between batches of the same product). The caffeine content of selected PSW ranged from 91 to $387 \mathrm{mg}$-serve $\mathrm{e}^{-1}$. Only six of the 15 PWS nutrition information panels included details on caffeine content. The percent of caffeine present ranged from $59 \%$ to $176 \%$ of packaging claims. All but one PWS contained a variation of caffeine within and between batches that was considered "practically" significant (i.e., $\geq 40 \mathrm{mg}$. serve ${ }^{-1}$ variation). Consumers are likely to be exposed to large and variable caffeine doses if ingesting PWS. Product information panels do little to improve consumer awareness of likely caffeine intakes.

2.106. The Prevalence and Nutrient Composition of Postexercise Supplements in Certified Recovery Products

S. Srikulnath 1,2, C.A. Gouws 1,3, A.J. McKune 1,3, N.M. D'Cunha 1,3, J. Williams 1,3 and N. Naumovski ${ }^{1,3}$

${ }^{1}$ Faculty of Health, University of Canberra, Bruce, ACT, Australia

2 Institute of Food Research and Product Development, Kasetsart University, Chatuchak, Bangkok, Thailand

${ }^{3}$ Collaborative Research in Bioactives and Biomarkers (CRIBB) group, Bruce, ACT, Australia

Athletes are consuming sports supplements to promote recovery from intense exercise. However, there is conflicting evidence surrounding the benefits of recovery supplements consumed in periods postexercise. This study aimed to identify the number and compositional characteristics of certified commercially available products by 'Informed-Sport' based on manufacturers suggested consumption during "post-(exercise, workout or training)" periods. A systematic search of the product websites $(n=739)$, identified $39(5.3 \%)$ products to use the wording of "post" on either 'front-of-label', 'product directions' or within the product website's description or instructions. Of these, 36 were identified as powders, two as bars and one as a gel product. The mean energy was $1453 \pm 282(\mathrm{~kJ} / 100 \mathrm{~g})$ for powders, $1553 \pm 217(\mathrm{~kJ} / 100 \mathrm{~g})$ for bars and $478(\mathrm{~kJ} / 100 \mathrm{~g})$ for the gel product. By macronutrient, the highest average protein content were in powders $(46.2 \pm 28.0 / 100 \mathrm{~g})$, while carbohydrates were most prevalent in bars $(26.5 \pm 12.0 / 100 \mathrm{~g})$. In total, 25 of the 36 powdered products were protein based supplements. The most common 'other ingredients' included glutamine $(n=22)$ and leucine $(n=20)$ across all products. Conclusively, powder-based products are the most prominent and 
most-marketed commercially available certified as safe postexercise recovery supplements. There is currently opportunity for development of certified postexercise bars and gels to enhance recovery.

\subsection{Higher Dietary Nitrate Intake is Associated with Better Muscle Function in Older Women}

M. Sim 1,2, J.R. Lewis 1,2,3, L.C. Blekkenhorst ${ }^{1,2}$, C.P. Bondonno ${ }^{1,2}$, A. Devine ${ }^{1}$, K. Zhu 2,4, P. Peeling 2,5, R.L. Prince ${ }^{2,4}$ and J.M. Hodgson ${ }^{1,2}$

${ }^{1}$ Edith Cowan University, WA, Australia

${ }^{2}$ The University Western Australia, WA, Australia

${ }^{3}$ The University of Sydney, NSW, Australia

${ }^{4}$ Sir Charles Gairdner Hospital, WA, Australia

${ }^{5}$ Western Australian Institute of Sport, WA, Australia

The impact of higher habitual nitrate intake on muscle function in ageing is unknown. The cross-sectional relationship between dietary nitrate and measures of muscle function in older Australian women ( $n=1420, \geq 70$ years) was investigated. Total nitrate (from vegetables and nonvegetable sources) was calculated from a validated food frequency questionnaire and nitrate instrument. Handgrip strength and timed-up-and-go (TUG) were assessed, representing muscle strength and physical function, respectively. Mean \pm SD total nitrate intake was $79.5 \pm 31.2 \mathrm{mg} / \mathrm{d}$, of which $85 \%$ was derived from vegetables. In separate multivariable-adjusted models, higher nitrate intake (per SD, $31.2 \mathrm{mg} / \mathrm{d})$ was associated with stronger grip strength $(\beta 0.31, p=0.027)$ and faster TUG ( $\beta-0.27, p=0.001)$. Each $31.2 \mathrm{mg} / \mathrm{d}(1 \mathrm{SD})$ higher nitrate intake was associated with lower odds for weak grip strength $(16 \%, p=0.005)$ and slow TUG $(14 \%, p=0.021)$. Compared to women in the lowest tertile of nitrate intake $(<64.2 \mathrm{mg} / \mathrm{d})$, women with higher $(\geq 89.0 \mathrm{mg} / \mathrm{d})$ nitrate intake had lower odds for weak grip strength $\left(35 \%\right.$, $\left.\mathrm{p}_{\text {trend }}=0.004\right)$ and slow TUG $\left(28 \%\right.$, $\left.\mathrm{p}_{\text {trend }}=0.044\right)$. Considering the positive relationship observed between dietary nitrate and muscle function, older individuals should incorporate nitrate-rich green leafy vegetables and beetroot into their diet regularly.

\subsection{A Recipe for Success: Key Ingredients for An Effective Nutrition Education Program for Parents of Young Children in Regional and Remote WA}

V.E. Blake and L. Thorne

Foodbank WA, Perth Airport, WA, Australia

Early childhood has been identified as a critical period for nutrition interventions, as developing healthy eating behaviours during the first five years of life reduces the burden of disease in adulthood. Nutrition interventions need to be aimed primarily at parents/caregivers, as the decision-makers of their child's food intake. Foodbank WA's innovative Food Sensations ${ }^{\circledR}$ for Parents (FSP) program delivers nutrition education and cooking workshops to disadvantaged parents of $0-5$ year olds living in the remote Pilbara region in WA. Three key elements underpin the structure of this unique program: a strong focus on relationship building; appropriate nutrition messaging; and interactive workshop delivery with children and parents cooking together. Since the program's inception in 2016, evaluation demonstrates the FSP program is a successful nutrition initiative. Prioritising relationship building with the target group develops trust and boosts engagement, while identifying appropriate nutrition messages ensures that workshop content is relevant and culturally appropriate. The interactive style of delivery supports hands-on learning as well as increases participants' skills and confidence. By applying the three key elements to FSP, parents complete the program with the knowledge and skills to set up their children with healthy eating behaviours for life. 
2.109. Secondary School Teachers' Perceptions of Healthy Eating: A Qualitative Exploration of Knowledge and Views

\author{
G.K. Boddy, A. Booth and A. Worsley
}

Deakin University, Burwood, VIC, Australia

Teachers disseminate food knowledge and skills in secondary school curricula that provide essential skills for a healthy life. Yet at present, teachers' perceptions of the place of healthy eating in curricula are unknown. Fifteen teachers' perceptions were explored by semistructured, in-depth interviews and thematic analysis. Their responses were grouped into five themes: healthy eating approaches, food information influences, curriculum planning, teaching goals and teacher career influences. Teachers were clear about the aims and importance of teaching healthy eating in an experiential curriculum. They reported that teaching healthy eating assists the health and wellbeing of adolescents and their families. Its effectiveness may be compromised by the current positioning of food, nutrition and health topics in two separate curriculum areas, Technologies and Health and Physical Education; and competing school priorities and resources that limit the students' exposures to food curricula. They sourced food information from online websites, popular culture and social media. Teachers' knowledge and views of healthy eating appeared to be associated with their life experiences, education and employment histories. These findings can assist with education policy development and the design of healthy eating curriculum approaches that will foster healthy food habits for adolescents, their families, now and in the future.

\title{
2.110. School Nutrition Education Programs in the Pacific Islands-An Overview of the Current Situation
}

S. Burkhart ${ }^{1}$, A. Hayman ${ }^{2}$, B. Jones ${ }^{1}$, F. Lam ${ }^{2}$, S. Underhill ${ }^{1}$ and E. Swanepoel ${ }^{1}$

${ }^{1}$ University of the Sunshine Coast, Maroochydore DC, QLD, Australia

${ }^{2}$ Food and Agriculture Organization of the United Nations, Subregional Office for the Pacific Islands

School-aged Pacific Island (PI) populations face considerable nutrition issues including the double burden of malnutrition. Schools provide an ideal setting to influence dietary behaviours of youth and the wider community; however, it is unclear if students attending PI schools are being educated on healthy nutrition practices. The aim of this study is to identify current School Nutrition Education Programs (SNEP) across 14 PI countries. A desk review was validated with semistructured stakeholder interviews (in person $n=12$ countries, via email contact $n=2$ countries), and email meeting summaries to identify SNEP currently in use. Thematic analysis of meeting summaries revealed three types of SNEP: curriculum; gardening; and other (sport-based school food). All PI countries had a formal education curriculum; however, nutrition was not explicitly included in two countries. The Health Promoting Schools Framework (WHO) is used in six PI countries, while all countries used garden based activities but with varied and limited curriculum integration. Formal sport related activities were used in two countries, and school food programs are currently used to varying extent in three countries. A range of SNEP's are currently used across the PI, with majority based on curriculum and gardening, however integration with the curriculum is limited.

\subsection{School Nutrition Education Programs in the Pacific Islands-A Capacity Needs Assessment}

S. Burkhart ${ }^{1}$, A. Hayman ${ }^{2}$, B. Jones ${ }^{1}$, F. Lam ${ }^{2}$, S. Underhill ${ }^{1}$ and E. Swanepoel ${ }^{1}$

${ }^{1}$ University of the Sunshine Coast, Maroochydore DC, QLD, Australia

${ }^{2}$ Food and Agriculture Organization of the United Nations, Subregional Office for the Pacific Islands, Bangkok, Thailand

Diverse School Nutrition Education Programs (SNEP) are currently used throughout the Pacific Islands (PI), however there is little understanding regarding the capacity of countries to develop and sustain these activities. The aim of this study was to undertake a Capacity Needs Assessment (CNA) 
of SNEP in 14 PI countries. A desk review was validated through semistructured interviews with 88 key stakeholders (in person $n=12$ countries, via email $n=2$ countries), and email verification of meeting summaries. Content analyses were guided by an adapted CNA Framework (FAO). There is high motivation for SNEP's, however only nine countries have an overarching food/nutrition/NCD policy, and eight PI a country-wide school policy. Stakeholders across 11 countries report requiring assistance to develop contextualised learning materials, and difficulty sourcing credible information for teaching. Partnerships and collaboration across sectors within each country varied with remoteness/geographical location, communication technology and funding cited as challenges, and the need for a formal regional PI stakeholder group (i.e., nutritionists, educators and agricultural experts) identified. Stakeholders reported facilities (i.e., garden space), human resources and funding limited implementation, including monitoring and evaluation. While high-level support and motivation exists, enhanced policy/normative, knowledge, partnering and implementation capacity would likely assist SNEP development and sustainability in this region.

\subsection{Health Star Rating in Grain Foods_-Does It Adequately Differentiate between Refined and Whole Grain Foods}

\section{F.J. Curtain and S.J. Grafenauer}

\section{Grains and Legumes Nutrition Council, North Sydney, NSW, Australia}

Australian Dietary Guidelines recommend mostly whole grain (WG) foods because of their higher fibre, vitamins, minerals and antioxidants compared to refined grains (RG). The Health Star Rating (HSR) front-of-pack labelling system ranks packaged foods from $\frac{1}{2}-5$ stars, based on an algorithm taking into account 'positive' and 'negative' ingredients (the former includes fruit, vegetables, nuts and legumes, but not WGs). In the context of the HSR 5-year review, this study aimed to determine whether HSR adequately differentiates WG and RG choices. Product data collected between 2016 and 18 through rolling audits $(n=1120)$ was analysed to calculate the mean and median HSR of RG and WG bread, rice, pasta, noodles, flour, breakfast cereals and bars. Overall 510 products displayed a HSR (45\%). Mean and median HSR was identical between RG and WG products, at 3.8 and 4 stars, respectively. Median HSR was also identical for RG and WG bread, bars, breakfast cereals and flour, while WG rice was higher than RG by $\frac{1}{2}$. No noodles or WG pasta displayed a HSR. The current HSR algorithm does not adequately reflect the nutritional benefits of WG products, and including WG in the algorithm would increase its sensitivity, maximising consumers' trust and ability to choose healthy grain foods.

\subsection{Analysis of Antioxidant Levels of Common Cooking Oils in Australia}

\section{F. De Alzaa}

Olive Wellness Institute, Lara, VIC, Australia

Despite the large variety of cooking oils in Australia, there is a lack of published data assessing level of antioxidants (biophenols, squalene and alpha-tocopherols) in these oils. Antioxidant levels are key predictors of the stability of oil to cook with at high temperatures. We assessed levels of antioxidants in six common cooking oils (extra virgin olive oil (EVOO), coconut, canola, grapeseed, rice bran and vegetable oils) from three major supermarket chains in the Melbourne-Geelong region, Australia. Analysis was performed in an ISO17025 accredited laboratory (2016-2018). On average EVOO antioxidant content was significantly higher $(4907.8 \mathrm{mg} / \mathrm{kg})$ compared with the other cooking oils $(\sim 268.6 \mathrm{mg} / \mathrm{kg})$, due to higher concentrations of squalene $(\sim 4330.1 \mathrm{mg} / \mathrm{kg}$ vs. $220.9 \mathrm{mg} / \mathrm{kg})$ and biophenols $(\sim 431.8 \mathrm{mg} / \mathrm{kg}$ vs. $1.6 \mathrm{mg} / \mathrm{kg})$ in EVOO. Concentration of alpha-tocopherols was highest in Canola oil $(\sim 149.4 \mathrm{mg} / \mathrm{kg})$ and EVOO $(\sim 146.9 \mathrm{mg} / \mathrm{kg})$. However, most alpha-tocopherols in seed oils and refined oils such as Canola oil are not naturally present but artificially added to increase the oil's shelf life. Virgin coconut oil contained the lowest level of antioxidants tested $(\sim 8 \mathrm{~kg} / \mathrm{mg})$ with no 
detectable squalene. These results show that EVOO contains the highest levels of natural antioxidants and is likely to be the most stable oil for high temperature cooking.

2.114. The Development and Design of a Multicontact Mode National Data Quality Web Survey for Clinical Research in Allied Health

L. Houston ${ }^{1,2}$ and Y. Probst ${ }^{1,2}$

1 Illawarra Health and Medical Research Institute, University of Wollongong, Wollongong, NSW, Australia

${ }^{2}$ School of Medicine, Faculty of Science, Medicine and Health, University of Wollongong, Wollongong, NSW, Australia

Allied health (AH) research is a multidisciplinary field of scientific enquiry to improve the overall health status. Surveys are a popular method for data collection though affected by low response rates $(\sim 10 \%)$ from single-mode designs. This research describes the development of a multicontact mode national survey. A tailored holistic approach was applied to minimise error. A social exchange theory was applied to decrease costs and increase benefits of responding and establish trust. Survey design was suited to the population (AH researchers), situation (Australia) and topic (data quality). Feasibility testing guided question development. Question wording, structure, order and grouping were reviewed using construct validation $(n=3)$ and expert review $(n=1)$. The survey was developed using the REDCap tool and further, think-aloud cognitive interviews $(n=5)$ identified wording, question order, visual design, presentation and navigation problems. Multicontact modes included a postal letter followed by three timed e-mail requests/reminders all including URL links to the survey. The national self-administered semiquantitate web survey was administered to 3835 participants over an 11-week period. The systematic survey design lowered costs, improved timeliness, improved response rate, reduced coverage and measurement error. The multiple modes provided an effective communication method by creating trust and legitimacy of research surveys in AH.

2.115. Nutrition Science Competency Standards for Australian Undergraduate Nutrition Science Degrees: A Joint NSA and ANT Initiative

T. Lawlis ${ }^{1}$, S. Torres ${ }^{2}$, A. Coates ${ }^{3}$, K. Clark ${ }^{4}$, K. Charlton ${ }^{5,6}$, A. Sinclair ${ }^{7,8}$, L. Wood ${ }^{9}$ and A. Devine ${ }^{10}$

${ }^{1}$ Faculty of Health, University of Canberra, Canberra, ACT, Australia

2 Institute for Physical Activity and Nutrition, School of Exercise and Nutrition Sciences, Deakin University, Geelong, VIC, Australia

${ }^{3}$ Alliance for Research in Exercise, Nutrition and Activity, School of Health Sciences, University of South Australia, Adelaide, SA, Australia

${ }^{4}$ School of Public Health, Curtin University, Perth, WA, Australia

${ }^{5}$ Australia and Illawarra Health and Medical Research Institute, Wollongong, NSW, Australia

${ }^{6}$ School of Medicine, University of Wollongong, Wollongong, NSW, Australia

${ }^{7}$ Faculty of Health, Deakin University, Geelong, VIC, Australia

${ }^{8}$ Department of Nutrition, Dietetics and Food, Monash University, Notting Hill, VIC, Australia

${ }^{9}$ School of Biomedical Science and Pharmacy, Faculty of Health and Medicine, University of Newcastle, Newcastle, NSW, Australia

${ }^{10}$ School of Medical and Health Sciences, Edith Cowan University, Perth, WA, Australia

This paper describes revision of the nutrition scientist professional competency standards for use in Australia. Nutrition experts from industry, community, government and academia $(n=128)$ were consulted to define areas required for nutrition science practice. The process used a multiple methods iterative approach consisting of three stages: (1) scoping study of existing competencies; (2) exploratory survey; and (3) a modified Delphi process (two rounds). After applying a $\geq 70 \%$ consensus rule, a final list of competency standards were identified; five core and three specialist competencies. The five core 
standards are defined as Nutrition Science, Food and the Food System, Nutrition Governance and Sociocultural and Behavioural Factors, Nutrition Research and Critical Analysis and Communication and Professional Conduct. These standards are all supported by fundamental knowledge, skills, attitudes and values. The three specialist competency standards are Food Science, Public Health Nutrition and Animal Nutrition. These new nutrition science competency standards acknowledge diversity within the science of nutrition. They describe the professional expectations of entry-level nutritionists and nutrition scientists for effective practice in the Australian workforce and provide a framework to benchmark Australian nutrition science degrees and majors with the aim of building professional identity and strengthening graduate outcomes.

\subsection{Nutrition Communication by the Chiropractic Profession}

M.K. Lee, L. Amorin-Woods and V. Cascioli

School of Health Professions, Murdoch University, Murdoch, WA, Australia

There are 5400 chiropractors registered to practice in Australia, $60 \%$ male and $40 \%$ female. This profession has had a long interest in promoting nutrition. The aim was to document the extent of nutrition promotion by chiropractors and their major sources of information.

A sample of 1000 practising chiropractors was randomly selected from the Australian Chiropractic Research Network (ACORN) database for a cross-sectional study. A total of 33\% participated in the online survey. When asked whether they provide nutritional advice to their patients on more than $50 \%$ of occasions, male chiropractors were less likely to do so (33\%) compared to $46 \%$ for females $\left(\mathrm{X}^{2} 6.04, p=0.014\right)$. A smaller number of practitioners $(30 \%)$ stated that providing nutritional advice was not a major component of their practice (i.e., 'never' or to 'less than $25 \%$ of patients'). Nutrition-related conditions most commonly encountered by the chiropractors were musculoskeletal, usually inflammatory in origin. While most of the respondents were familiar with the Australian Dietary Guidelines (70\%) only a small proportion (17\%) reported regularly using the Australian Guide to Healthy Eating when providing nutritional guidance to patients. Australian chiropractors acknowledge the importance of nutrition in their clinical practice especially for patients presenting with chronic disease.

\subsection{Understanding the Aspects of An Engaging Social Media Post}

E. Herron ${ }^{1}$, A. Molenaar ${ }^{1}$, J. Choong ${ }^{1}$, K. Klassen ${ }^{1}$, M. Lim ${ }^{2}$, L. Brennan ${ }^{3}$, M. Reid ${ }^{4}$, H. Truby ${ }^{1}$ and T. McCaffrey ${ }^{1}$

${ }^{1}$ Department of Nutrition, Dietetics and Food, Monash University, Notting Hill, VIC, Australia

2 Behaviours and Health Risks, Burnet Institute, Melbourne, VIC, Australia

${ }^{3}$ School of Media \& Communication, RMIT University, Melbourne, VIC, Australia

${ }^{4}$ School of Economics, Finance and Marketing, RMIT University, Melbourne, VIC, Australia

People are increasingly using social media (SM) to seek health and nutrition information. SM personalities have high engagement and influence over followers, something that nutrition and health professionals often lack. We aimed to identify SM strategies associated with high public engagement across food industry $(n=10)$, lifestyle personalities $(n=10)$, health organisations $(n=10)$ and nutrition professionals $(n=10)$. An exploratory content analysis using a coding framework was conducted on 524 posts from Instagram, Facebook and Twitter. The association between post strategies and engagement metrics (likes, shares etc.) was explored using multivariate linear regression. Engagement was higher for lifestyle personalities on all platforms. On Facebook, Food Industry posts frequently used "visually appealing" strategies (13\%) and health organisation frequently posted "stories" $(8 \%)$. Strategies most positively associated with engagement were "people appearing in posts" on Instagram $(\beta$ 0.233, $p<0.001)$ and a "positive tone" on Twitter $(\beta 0.218, p<0.009)$. Strategies negatively associated with engagement were "association with health success" on Facebook 
( $\beta=-0.208, p<0.002)$, Instagram $(\beta=-0.228, p<0.001)$ and on Twitter, those with "links to health information" ( $\beta=-0.251 p<0.001)$. Nutrition professionals and health organisations could learn from successful engagement strategies of online influencers as to how to increase reach of their messages.

2.118. Farm to Fork: A Nexus between School Curriculum, Adolescent Interests and Game Design for Nutrition Education Engagement

M. Miller ${ }^{1}$, M. Masek ${ }^{2}$, J. Boston ${ }^{3}$, M. Furfaro ${ }^{1}$, D. Barwood ${ }^{3}$, S. Smith ${ }^{3}$ and A. Devine ${ }^{1}$

${ }^{1}$ School of Medicine and Health Sciences, Edith Cowan University, Perth, WA, Australia

${ }^{2}$ School of Science, Edith Cowan University, Perth, WA, Australia

${ }^{3}$ School of Education, Edith Cowan University, Perth, WA Australia

The Australian Curriculum provides new opportunities for food and nutrition education but engaging high school students and teachers can be challenging. This research used an electronic game design project to assess what topics would appeal to adolescents and integrate with curriculum. In focus groups, 68 boys and girls, years 7-10, across social strata in WA contributed ideas about topics then created related game scenarios. Seventeen scenarios across seven topics were curriculum mapped in workshops with 29 teachers from three learning areas. A game decision matrix was applied to capture student interests, curriculum outcomes and effective design in a prototype game pilot-tested in 17 classrooms. Two distinct themes emerged as top interests "Providing food for needy people to reduce hunger" and "Eating the right foods for keeping healthy, active and healthy weight". Reducing food waste, food safety and food production were also raised. Capturing these, the prototype game design features the journey of a food commodity from production to consumer with content that addresses learning outcomes for Science, Health, Technologies and others. The pilot provided evidence of student engagement and curriculum-relevant learning and enquiry. We conclude, knowing student interests and teacher curriculum priorities is the nexus for nutrition education resource design.

\subsection{Mothers' Perceptions of Toddler Beverages}

M. Rigo ${ }^{1}$, J. Willcox ${ }^{2}$, A. Spence ${ }^{1}$ and A. Worsley ${ }^{1}$

${ }^{1}$ Deakin University, Burwood, VIC, Australia

${ }^{2}$ Health, Latrobe University, Bundoora, VIC, Australia

The prevalence of obesity among Australian preschool children is a major health concern. Sugar-sweetened beverages are energy-dense, nutrient-poor and readily available and have been implicated in the increasing prevalence of obesity. Furthermore, consumption at a young age may result in lifetime habits. There is little research on factors influencing parents' decision making when serving beverages to their preschools, or on mothers' perceptions of these beverages.

To explore mothers' perceptions of toddler beverages, qualitative interviews were conducted with 28 mothers from Melbourne using the Repertory Grid Technique and Laddering Technique methodologies. A large number of diverse perceptual categories ('constructs') $(n=22)$ about beverages were elicited, demonstrating the complexity of mother's perceptions when making beverage choices for their preschools. The five most common categories were related to health, sugar, dairy, packaging and additives. Thematic analysis of responses from the laddering method identified three major themes: concerns about the types of beverages mothers would like to provide their preschools; the healthiness of a beverage; and the sugar content.

In conclusion, mothers' perceptions of beverages are sophisticated and should be considered in the design of health communication strategies to influence mothers' dietary choices for their preschools. 
2.120. Towards Effective Nutrition Education for Dutch Primary School Children: An Assessment of Effective Components

A. Verdonschot ${ }^{1,2}$, T. Bucher ${ }^{1}$, C. Collins ${ }^{1}$, E. de Vet $^{2}$ and A. Haveman-Nies ${ }^{2}$

${ }^{1}$ Faculty of Health and Medicine, University of Newcastle, Newcastle, NSW, Australia

2 Strategic Communication Group, Wageningen University \& Research, Wageningen, Gelderland, The Netherlands

A healthy diet is important for children's growth and development. Children need encouragement and support to adopt healthy eating behaviours. Dutch nutrition education programs such as EU-Schoolfruit (EUS) and Taste Lessons (TL) contribute to this by providing children with fruit, vegetables (FV) (EUS) and nutrition education (TL). However, little is known about which components are most effective. It is hypothesised that children participating in nutrition education programs that implement both environmental and educational components will have greater nutrition knowledge, food literacy and higher FV intakes, compared to children participating in nutrition education programs with one component. This research examines effective components of existing Dutch nutrition education programs for primary school children ( $n=2250, n=45$ schools) aged 9-11 years. Child nutrition knowledge, food literacy and FV intakes will be measured by questionnaire. This quasi-experimental study has three arms: (1) schools that implement EUS, (2) schools that implement EUS + TL and (3) schools that implement no nutrition education. Outcomes will be assessed pre-intervention (baseline), immediately following the intervention (postintervention), and 6 months postintervention. The study protocol and preliminary results are presented. The results can contribute to the design of future effective nutrition education programs, which support addressing the problem of childhood obesity.

\subsection{Development of a Dietary Guideline Index for Application to Weighed Food Records}

S.J. Ward ${ }^{1}$, A.M. Coates ${ }^{1}$ and A.M. Hill ${ }^{2}$

${ }^{1}$ School of Health Sciences, University of South Australia, Adelaide, SA, Australia

${ }^{2}$ School of Pharmacy and Medical Sciences, University of South Australia, Adelaide, SA, Australia

There has been increased interest in methodologies to analyse whole diets as the quality of the overall diet has been associated with health and disease risk. The Dietary Guideline Index (DGI) is a validated diet quality index that reflects adherence to the Australian Dietary Guidelines (ADG). To date, composition of the DGI has been based on food frequencies and quantities through preestablished servings. The aim of the current study was to develop a methodology that applied the DGI to dietary intake data collected via gold standard, weighed food records. The DGI was applied to a convenience sample of 133 adults aged 50-80 years (80 female, 53 male). Consisting of 10 components, the DGI reflected variety and quality of ADG core food groups and discretionary choices. Mean DGI score was 50.7 out of a possible 100 (SD 12.3); less than half of participants met guidelines for variety, fruit, vegetables, lean meat and alternatives, dairy and unsaturated spreads/oils. All participants exceeded the limit for discretionary intake, with participants averaging 4.3 serves per day (SD 2.6). This methodological approach of applying the DGI to weighed food records may improve our ability to quantify diet quality.

\subsection{What Is The Impact of AMY1 Copy Number Variation on Interindividual Variability in Glycaemic} Index Testing

F.S. Atkinson ${ }^{1}$, D. Hancock ${ }^{1}$, P. Petocz ${ }^{2}$ and J.C. Brand-Miller ${ }^{1}$

${ }^{1}$ The University of Sydney, Camperdown, NSW, Australia

${ }^{2}$ Macquarie University, North Ryde, NSW, Australia 
Interindividual variation may affect the reliability and reproducibility of the glycaemic index (GI). Previously, we showed that salivary amylase gene (AMY1) copy number (CN) variation influences starch digestion and postprandial responses to starchy foods. AMY1 CN may therefore contribute to interindividual variation in the measurement of GI. We hypothesised that the impact of AMY1 CN variation on GI measurement could be managed through the use of a starch-based reference food. In a pool of healthy individuals with known AMY1 CN, the GI values of five starchy foods were determined relative to a glucose solution and white bread. When the reference food was glucose, the absolute GI values for the five foods were significantly higher in individuals with higher compared to lower AMY1 CN (all foods $p<0.05$ ). However, when white bread was the reference, there were no significant differences in GI values between the two groups at the extremes of AMY1 CN. Irrespective of the reference food, AMY1 CN did not affect the relative GI ranking of the foods. Use of white bread as the reference food can reduce the influence of interindividual variation in AMY1 CN on the measurement of GI values for starchy foods.

\subsection{Polymorphisms in The NADSYN1/DHCR7 Locus as Potential Regulators of Interethnic Differences in} Vitamin D Production

P. Jones ${ }^{1}$, M. Lucock $^{1}$, M. Veysey ${ }^{2}$ and E. Beckett ${ }^{1,3}$

${ }^{1}$ School of Environmental \& Life Sciences, University of Newcastle, Ourimbah, NSW, Australia

${ }^{2}$ Hull-York Medical School, University of York, Heslington, York, UK

${ }^{3}$ School of Medicine \& Public Health, University of Newcastle, Ourimbah, NSW, Australia

The relationship between ultraviolet radiation (UVR) and vitamin D status is influenced by skin pigmentation and genetic variance in vitamin $\mathrm{D}$ pathways. There is evidence of association between these factors, potentially as an adaptation to reduced vitamin D production in highly-pigmented skin. Polymorphisms in 7-dehydrocholesterol reductase (DHCR7) and nicotinamide adenine dinucleotide synthetase (NADSYN1) are determinants of vitamin D status, but associations between this locus and skin type remains unexplored. Therefore we examined for association between frequency of common variants in DHCR7/NADSYN1 and skin pigmentation. Population prevalence of common DHCR7/NADSYN1 variants (minor allele frequency $\geq 0.35$ ) were collated from public genotypic databases (Allele Frequency Database and 1000 Genomes; $>100$ cohorts, 5000 subjects) and examined against population skin phototype (common Fitzpatrick phototype of reported population ancestry). Carriage of 10 variants-rs12797951, rs1790324, rs1630498, rs11603330, rs3750997, rs7928249, rs7944926, rs12800438, rs1792226 and rs3794060-were significantly associated with Fitzpatrick skin type ( $p<0.0001$ in all cases). Notably, increases in pigmentation were associated with occurrence increases in rs7944926 $\left(\mathrm{r}^{2} 0.66\right)$ and rs7928249 $\left(\mathrm{r}^{2} 0.52\right)$, and decreases in rs3794060 $\left(\mathrm{r}^{2} 0.67\right), \mathrm{rs} 11603330$ $\left(r^{2} 0.58\right)$ and $r s 12797951\left(r^{2} 0.54\right)$. Findings identify novel vitamin D-related genetic factors that may play part in differences in vitamin D UVR-production between ethnicities.

\subsection{DHA-Enriched Fish Oil Supplementation Reduces Insulin Resistance in Men and Women with} Abdominal Obesity: A Double-Blind Randomised Controlled Trial

K. Abbott ${ }^{1}$, T. Burrows ${ }^{2}$, S. Acharya ${ }^{3}$, R. Thota ${ }^{1}$ and M. Garg ${ }^{1}$

${ }^{1}$ Nutraceuticals Research Program, University of Newcastle, Callaghan, NSW, Australia

${ }^{2}$ School of Health Sciences, University of Newcastle, Callaghan, NSW, Australia

${ }^{3}$ Department of Endocrinology, John Hunter Hospital, Newcastle, NSW, Australia

The cause-consequence relationship between higher omega-3 polyunsaturated fatty acids levels and reduced insulin resistance (IR) is not understood. This study aimed to determine whether docosahexaenoic acid (DHA)-enriched fish oil supplementation reduces IR in overweight/obese adults. Participants were recruited if they were aged 18-70 years, had a waist circumference $>88 \mathrm{~cm}$ (female) or $>102 \mathrm{~cm}$ (male) and did not have diabetes. Participants were randomised to consume DHA-enriched 
fish oil (FO, $860 \mathrm{mg} /$ day) or placebo (corn oil, CO) capsules for three months. Fasted blood samples were collected at baseline and follow-up. Fasting glucose and insulin values were used to calculate HOMA-IR. Sixty-eight (FO, $n=36$; CO, $n=32$ ) participants completed the study (age, $50.9 \pm 12.7$ years; BMI, $32.4 \pm 6.6 \mathrm{~kg} / \mathrm{m}^{2}$; WC, $\left.103.4 \pm 14.8 \mathrm{~cm}\right)$. After adjusting for baseline values, FO decreased fasting insulin (mean difference [95\% CI]: $-1.62 \mu \mathrm{IU} / \mathrm{L}[-2.99,-0.26], p=0.021$ ) and HOMA-IR $(-0.40[-0.78,-0.02], p=0.038)$ compared with $\mathrm{CO}$, with no change in fasting glucose. Changes in insulin and HOMA-IR in FO group were inversely correlated with baseline values $(\mathrm{r}=-0.708$, $p<0.0001$ and $\mathrm{r}=-0.686, p<0.0001$, respectively). DHA-enriched fish oil supplementation reduced IR in overweight/obese individuals with hyperinsulinemia and IR, and may prove to be a useful adjunct treatment to lifestyle interventions for prevention of type 2 diabetes.

2.125. Associations between Long-Chain Omega-3 Polyunsaturated Fatty Acid Status and Diabetes, Non-Alcoholic Fatty Liver Disease and Metabolic Syndrome: The Hunter Cohort Study

K. Abbott ${ }^{1}$, T. Burrows ${ }^{2}$, R. Thota ${ }^{1}$, S. Acharya ${ }^{3}$, A. Alex $^{1}$ and M. Garg ${ }^{1}$

${ }^{1}$ Nutraceuticals Research Program, University of Newcastle, Callaghan, NSW, Australia

${ }^{2}$ School of Health Sciences, University of Newcastle, Callaghan, NSW, Australia

${ }^{3}$ Department of Endocrinology, John Hunter Hospital, Newcastle, NSW, Australia

Chronic subclinical inflammation is associated with obesity-induced insulin resistance, leading to metabolic syndrome (MetS), non-alcoholic fatty liver disease (NAFLD) and type 2 diabetes (T2D). Long-chain omega-3 polyunsaturated fatty acids (LCn-3PUFAs) are anti-inflammatory, thus may protect against obesity-related metabolic conditions. This study aimed to determine whether LCn-3PUFA status was associated with T2D, NAFLD or MetS in the Hunter Cohort Study. LCn-3PUFA status was determined according to plasma phospholipid composition (EPA + DHA, \% w/w); T2D defined by self-reported diagnosis or fasting glucose $>7.0 \mathrm{mmol} / \mathrm{L}$, NAFLD determined using Fatty Liver Index (FLI $>60)$ and MetS according to IDF Criteria. In total, $n=2092$ participants were included (age: $66.3 \pm 7.5$ years; BMI: $28.8 \pm 5.0 \mathrm{~kg} / \mathrm{m}^{2}$; female: $n=1059,50.6 \%$ ). After adjusting for known risk factors (age, sex, BMI, fibre intake and physical activity), LCn-3PUFA status was inversely associated with MetS (OR [95\% CI]: 1.00 [reference], 0.54 [0.37, 0.79], 0.58 [0.40, 0.85], 0.36 $[0.25,0.53], p$-trend $<0.001$ for first to fourth quartiles, respectively) and NAFLD (OR [95\% CI]: 1.00 [reference], 0.65 [0.49, 0.87], 0.47 [0.35, 0.63], 0.34 [0.25, 0.46], $p$-trend $=0.004)$, however T2D was not associated with LCn-3PUFA (OR [95\% CI]: 1.00 [reference], 0.69 [0.41, 1.14], 0.42 [0.24, 0.75], 0.85 [0.52, $1.40], p$-trend $=0.293)$. LCn-3PUFA status is inversely associated with MetS and NAFLD. Dietary LCn-3PUFAs may protect against obesity-related metabolic dysfunction.

\subsection{Effect of Maternal Dietary Glycaemic Index on Offspring Metabolic Programming in Mice}

G.J. Campbell, S.G. Lucic Fisher and K.S. Bell-Anderson

Charles Perkins Centre and School of Life and Environmental Sciences, University of Sydney, Sydney, NSW, Australia

The glycaemic index (GI) is a measure of carbohydrate quality with regards to its effect on blood glucose levels, with low GI diets being associated with improved metabolic health in humans and rodents. However, present research is unable to elucidate the mechanism(s) responsible. Isocaloric rodent diets differing only in the base sugar carbohydrate (high GI: glucose, sucrose; low GI: isomaltulose, fructose) were constructed such that fibre content was controlled, but GI varied. Embryos and pups of C57BL/6 mothers fed these diets or chow were compared. Embryos of chow-fed mothers were significantly heavier than embryos from glucose and isomaltulose-fed mums and had significantly heavier livers and spleens than all sugar embryos. Male pups were heavier, leaner and more glucose-intolerant than females across all diets. Differences were observed only in male pups. Chow, glucose and sucrose male pups were heavier than isomaltulose and fructose pups. Fructose 
and glucose male pups had higher insulin peaks at 12 weeks of age, while there was no difference in basal blood glucose levels. Maternal dietary sugars exhibit a limited effect on offspring metabolic health, but only in male pups. Compared with chow, the sugar diet pups were lighter, fatter and less glucose tolerant.

\subsection{Body Composition Changes during a 6-Month Modified Fasting Intervention for Adults Newly} Diagnosed with Obstructive Sleep Apnoea

K. Day ${ }^{1}$, H. Truby ${ }^{1}$, B. Edwards ${ }^{1}$, D. O'Driscoll ${ }^{1,2}$, A. Young ${ }^{2}$, L. Ghazi ${ }^{1}$, C. Bristow ${ }^{1}$, K. Roem ${ }^{1}$, M. Bonham ${ }^{1}$, C. Murgia ${ }^{1}$, T. Haines ${ }^{1}$ and G. Hamilton ${ }^{1,3}$

${ }^{1}$ Monash University, Melbourne, VIC, Australia

${ }^{2}$ Department of Respiratory and Sleep Medicine, Eastern Health, Box Hill, VIC, Australia

${ }^{3}$ Department of Lung and Sleep, Monash Medical Centre, Clayton, VIC, Australia

Obstructive Sleep Apnoea (OSA) shares a complex relationship with obesity with some evidence that greater compliance with treatment (continuous positive airway pressure, CPAP) leads to weight gain. Modified fasting (MF) may offer an alternative method of weight loss. Participants $(n=59$, mean body mass index $34.1 \mathrm{~kg} / \mathrm{m}^{2} \pm 4.7 \mathrm{~kg} / \mathrm{m}^{2}$ ) underwent a six month MF intervention concurrent with CPAP treatment. The intervention consisted of 3-months active weight loss followed by 3-months weight maintenance. Moderate physical activity was encouraged. At baseline and postintervention weight, waist circumference and body composition were measured. Data were analysed using paired $t$-test (SPSS, v.25). On average, participants lost $-5.0 \mathrm{~kg} \pm 6.5 \mathrm{~kg}$ body weight $(p<0.001)$ and reduced waist circumference by $-5.4 \mathrm{~cm} \pm 5.9 \mathrm{~cm}$. Body fat $(-5.4 \mathrm{~kg}$ ) and visceral adipose tissue (VAT, $-0.6 \mathrm{~kg}$ ) were significantly reduced after six months of MF $(p<0.001)$ with conservation of fat free mass (FFM, $\Delta 0.0 \mathrm{~kg}, p=0.064)$. Apnoea Hypopnoea Index decreased by $8.4 \pm 18.8$ events $/ \mathrm{h}(p<0.01)$. MF can result in beneficial changes in body composition, comprising mainly of loss of fat, including VAT, with a conservation of FFM and provides a useful alternative to continuous energy restriction for adults starting CPAP therapy.

\subsection{Nutritional Adequacy of A Very-Low-Energy Diet for Weight Loss in Children and Adolescents}

\section{M.L. Gow ${ }^{1,2}$, H.Y.J. Wong ${ }^{3}$, H. Jebeile ${ }^{1,2}$, S.P. Garnett ${ }^{1,2}$ and N. Lister ${ }^{1,2}$}

1 Children's Hospital at Westmead Clinical School, The University of Sydney, Westmead, NSW, Australia

2 The Institute of Endocrinology and Diabetes, The Children's Hospital at Westmead, Westmead, NSW, Australia

${ }^{3}$ Nutrition and Dietetics Group, School of Life and Environmental Science, The Charles Perkins Centre, The University of Sydney, NSW, Australia

Very-low-energy diets (VLEDs) achieve rapid weight loss. However, their safety and acceptability for young people has not been adequately examined. This study addresses aspects of VLED safety and acceptability in children and adolescents by assessing (1) nutritional adequacy, (2) adherence and (3) changes in appetite hormones. This is secondary analysis of the SHAKE-IT pilot study which recruited eight 7-16 years olds with obesity and type 2 diabetes. Participants completed an 8-week VLED (800 kcal/day) and 26-week follow-up. Nutritional adequacy (compared to Australian nutrient reference values [NRVs]) and dietary adherence ( $\leq 800 \mathrm{kcal} /$ day) were assessed using 24 -h recalls available from six participants. Ghrelin and peptide $Y Y$ concentrations were assessed from fasting blood at baseline and 8- and 34-weeks. Compared to baseline, more participants met the NRVs during VLED for riboflavin, iron, magnesium, selenium, zinc and vitamins A, B6, B12 and C. Fewer met NRVs for fibre, folate, phosphorus and sodium. The NRV for calcium was not met by any participant at any time. Only $1 / 3$ participants reported VLED adherence. Ghrelin and peptide YY concentrations did not change. VLED improves nutrient profile compared with usual intake. Nutritional adequacy would 
be enhanced by improved adherence. Expected changes in appetite hormones to facilitate adherence were not observed.

\subsection{Dietary Sodium Intake and Adiposity in Adults: A Systematic Review and Meta-Analysis}

C.A. Grimes ${ }^{1}$, A.B. Booth ${ }^{1}$, K.B. Bolton ${ }^{1}$, K. Lim ${ }^{1}$, C. Service ${ }^{1}$, A. Khokhar ${ }^{1}$, F.J. He ${ }^{2}$ and C.A. Nowson ${ }^{1}$

${ }^{1}$ Deakin University, Melbourne, VIC, Australia

2 Wolfson Institute of Preventive Medicine, Queen Mary University of London, London, UK

Epidemiological evidence and some animal studies indicate higher intakes of sodium may contribute to weight gain. A systematic literature search was performed identifying cross-sectional and longitudinal ( $\geq 1$ year) studies that measured sodium intake and indices of adiposity; randomised controlled trials (RCTs) reduced dietary sodium ( $\geq 3$ months). Meta-analysis was performed for adiposity outcomes with $\geq 3$ studies. Mean difference (MD) or odds ratios (OR) associated with higher sodium intakes (lowest vs. highest percentile) and net change in body weight (BW) between reduced sodium and control diet were assessed. Forty-seven cross-sectional, four longitudinal and 16 RCTs were included. Cross-sectionally, higher sodium intakes were associated with overweight/obesity (three studies; $n=10,923 ;(\mathrm{OR}) 1.83,95 \%$ CI: 1.40, 2.43) and higher waist circumference (eight studies; $n=32,960 ; \mathrm{MD}=5.51 \mathrm{~cm}, 95 \% \mathrm{CI}: 4.39,6.64)$. Methods were too mixed to pool longitudinal studies. The RCTs indicated a trend for lower BW on reduced sodium compared to control diets (15 trials; $n=4,291 ;-0.27 \mathrm{~kg}, 95 \% \mathrm{CI}:-0.55,0.01 ; p=0.06)$. Cross-sectional evidence indicates sodium intake was positively associated with adiposity and there was a trend suggesting adopting a lower sodium diet could result in a small amount of weight loss. Evidence from longitudinal studies was inconclusive.

2.130. Dietary Sodium Intake Is Positively Associated with Adiposity in Children: A Systematic Review and Meta-Analysis of Observational Studies

C.A. Grimes ${ }^{1}$, A.B. Booth ${ }^{1}$, K.B. Bolton ${ }^{1}$, K. Lim ${ }^{1}$, C. Service ${ }^{1}$, A. Khokhar ${ }^{1}$, F.J. He ${ }^{2}$ and C.A. Nowson ${ }^{1}$

${ }^{1}$ Deakin University, Melbourne, VIC, Australia

2 Wolfson Institute of Preventive Medicine, Queen Mary University of London, London, UK

Some studies have reported on associations between sodium intake and adiposity in children but no systematic review has been completed. A systematic search of four databases was conducted. Random effects meta-analysis was performed for adiposity outcomes with $\geq 3$ studies. Eighteen cross-sectional and two longitudinal studies were included in the systematic review. Six of the seven studies measuring BMI/BMI z-score and all seven studies measuring waist circumference reported significant positive associations of sodium intake with BMI/BMI z-score and waist circumference. Discrepancy across studies permitted only one meta-analysis (three cross-sectional studies, $n=3625$ ); findings indicated that higher sodium intake (highest vs. lowest percentile) was associated with overweight/obesity (odds ratio 3.29, 95\% CI: 2.25, 4.80). Longitudinal findings were mixed; one study reported increased sodium intake did not change odds of obesity over a 3-year period and one reported that baseline sodium intake $(1 \mathrm{~g} / \mathrm{d})$ predicted 5-year change in percent body fat $(\mathrm{BF})(+1.598 \% \mathrm{BF}$, $p<0.05)$. Evidence from cross-sectional studies among children indicates that higher sodium intakes were associated with higher body mass and central adiposity. Findings from longitudinal studies are limited and mixed. More longitudinal and RCTs are required to confirm these findings. 


\subsection{Intermittent Energy Restriction: Nutritional Adequacy and Diet Quality in Adolescence}

E. House ${ }^{1}$, H. Jebeile ${ }^{2,3}$, M. Gow ${ }^{2,3}$, S. Garnett ${ }^{3}$ and N. Lister ${ }^{2,3}$

${ }^{1}$ The University of Sydney, Nutrition and Dietetics Group, School of Life and Environmental Sciences, The Charles Perkins Centre, University of Sydney, Sydney, NSW, Australia

2 The University of Sydney, Children's Hospital Westmead Clinical School, Westmead, NSW, Australia

3 The Institute of Endocrinology and Diabetes, The Children's Hospital at Westmead, Westmead, NSW, Australia

Novel dietary interventions to treat obesity in adolescents are required to improve adherence and long-term outcomes. Intermittent Energy Restriction (IER) may appeal to adolescents due to its flexibility, however, little is known about nutrient adequacy and adherence. This secondary analysis examined nutritional adequacy, diet quality and adherence in 22 adolescents with obesity who participated in a 26-week IER pilot study. Nutrient intake and diet quality was determined from 24-h recalls and adherence to the 2510kJ "fasting" day energy prescription determined from estimated food records. Compared to baseline, reduced intake of nutrients including magnesium (week 8: mean difference \pm SEM: $71 \pm 26 \mathrm{mg} / \mathrm{d}, p=0.023, n=11$ ), phosphorus (week 8: $421 \pm 161 \mathrm{mg} / \mathrm{d}$, $p=0.026, n=11$ ) and dietary fibre (week 12: $7.9 \pm 1.9 \mathrm{~g} / \mathrm{d}, p=0.004, n=8$ ) was observed. Proportional to energy intake, micronutrient density increased for iron (week 8, 12, 26: 0.3-0.4 mg/1000 $\mathrm{kJ} / \mathrm{d}, p<0.05$ ), potassium (week 8, 12, 26: 85-101 mg/1000 kJ/d), phosphorus (week 12, 26: $43-48 \mathrm{mg} / 1000 \mathrm{~kJ} / \mathrm{d}, p<0.05$ ) and zinc (week $8,12: 0.5-0.6 \mathrm{mg} / 1000 \mathrm{~kJ} / \mathrm{d}, p<0.05$ ). Diet quality was unchanged. Participants reported consistent adherence to the energy prescription. Results suggest IER adherence is well maintained in adolescents with obesity, and the intervention may improve nutrient density. However, achieving nutritional adequacy during severe energy restriction was difficult and use of supplementation may be indicated.

\subsection{Low Glycaemic Index Diets as An Intervention for Obesity: A Systematic Review and Meta-Analysis}

K.E. Mills ${ }^{1}$, M.I. Zafar ${ }^{2}$, J. Zheng ${ }^{2}$, M.-M. Peng ${ }^{2}$ and L.-L. Chen ${ }^{2}$

${ }^{1}$ Science and Technology, University of Canberra, Canberra, ACT, Australia

2 Endocrinology, Union Hospital, Tongji Medical College, Huazhong University of Science and Technology, Wuhan, China

Low glycaemic index (GI) diets may aid in weight loss by reducing postprandial blood glucose excursions, leading to more stable blood glucose concentrations and therefore a reduction in hunger. To test this hypothesis, we conducted a systematic review and meta-analysis of randomised controlled trials comparing a low GI diet with other diet types. We included 101 studies involving 109 study arms and 8527 participants. We meta-analysed the studies using a random-effects model, and conducted subgroup analyses and meta-regression based on control diet, blood glucose control, baseline BMI and dietary GI. A low GI diet resulted in small but significant improvements in body weight, BMI, LDL and total cholesterol overall, although no individual control diet was significantly different from a low GI diet. Studies in people with normal blood glucose who achieved a difference in GI of 20 points or more resulted in a larger reduction in body weight (SMD $=-0.26 ; 95 \% \mathrm{CI}:-0.43,-0.09)$ and total cholesterol (SMD $=-0.24 ; 95 \%$ CI: $-0.42,-0.05$ ) than studies that only achieved a smaller reduction in GI. Low GI diets, especially diets achieving a substantial decrease in GI, were moderately effective in lowering body weight. 
2.133. Impact of Walnut Supplementation on Dietary Change for Weight Loss: A Secondary Analysis of the HealthTrack Randomised Controlled Trial

E.P. Neale ${ }^{1,2}$, L.C. Tapsell ${ }^{1,2}$, A. Martin ${ }^{1,2}$, M. Batterham ${ }^{3}$, C. Wibisono ${ }^{1}$ and Y. Probst ${ }^{1,2}$

${ }^{1}$ School of Medicine, University of Wollongong, Wollongong, NSW, Australia

2 Illawarra Health and Medical Research Institute, University of Wollongong, Wollongong, NSW, Australia

3 Statistical Consulting Service, School of Mathematics and Applied Statistics, University of Wollongong, Wollongong, NSW, Australia

Being more specific about individual food choices may be advantageous for weight loss. Including a healthy food within an overall diet plan may help to expose the effects of individual foods. This study aimed to examine the impact of including walnuts as a food supplement in diets for weight loss. We conducted a secondary analysis of the HealthTrack lifestyle intervention trial. Overweight and obese participants were randomised to usual care (C), interdisciplinary intervention including individualised dietary advice (I) or interdisciplinary intervention including $30 \mathrm{~g}$ walnuts/day (IW). Changes in body weight, energy intake and intake of key foods over three and 12 months were assessed. Data was available for $n=293$ participants after three months and $n=175$ after 12 months. After three months the IW group achieved the greatest weight loss ( $p=0.012$, compared to C) and reported greater improvements in healthy core food choices such as fruit, and decreases in discretionary foods/beverages, compared to $C(p=0.043$ and $p=0.022$, respectively). Weight loss remained greatest in IW at 12 months ( $p=0.028$ compared to $C$ ). This study has demonstrated that including a core food such as walnuts in an individualised diet plan may enhance desired dietary changes and improve weight loss.

\subsection{The Association between Food Liking and Diet Quality in a Young Victorian Adult Cohort}

H. Pnosamy, K. Livingstone, L. Riddell and S. Cicerale

School of Exercise and Nutrition Sciences, Deakin University, Melbourne, VIC, Australia

How much a food is liked or disliked has been shown in a small number of studies to be associated with choice of particular foods. Individuals with an increased liking for fatty, sweet and salty foods tend to consume them in greater amounts compared with those with a decreased liking. No studies have investigated food liking and its relationship with an overall measure of dietary nutritional adequacy (diet quality). Hence, the aim of the current study was to investigate the association between food liking and diet quality. Participants were recruited from Deakin University, Melbourne. An online questionnaire was used to collect participants' demographics, food liking and food consumption information. Pearson correlations were used to investigate the association between food liking and diet quality. Participants $(n=1962$, age $=21.3 \pm 23.3$, female $=74.8 \%$ ) completed the study. The findings demonstrated small-medium, significant, positive relationships between the liking of core foods (grouped together) $(\mathrm{r}=0.15, p<0.001)$, grains $(r=0.30, p<0.001)$, vegetables $(\mathrm{r}=0.21, p<0.001)$ and fruits $(r=0.18, p<0.001)$ and diet quality. Small, significant, negative relationships were also noted between the liking of discretionary foods (grouped together) $(\mathrm{r}=-0.23, p<0.001)$, animal proteins $(\mathrm{r}=-0.12, p<0.001)$, dairy products $(\mathrm{r}=-0.05, p<0.001)$ and diet quality. In conclusion, associations between the liking of core and discretionary foods and diet quality were found, although the relationships were small.

\subsection{Asthma Medication Use in Obese and Healthy Weight Asthmatics: A Systematic Review and Meta-Analysis}

C. Thompson, S. Eslick, B. Berthon and L. Wood

Centre for Healthy Lungs, Hunter Medical Research Institute, University of Newcastle, Callaghan, NSW, Australia 
Obesity is a common comorbidity in asthma which is associated with poorer asthma control, more frequent and severe exacerbations and reduced response to asthma pharmacotherapy. This review examines the differences in asthma medication use between obese and healthy weight asthmatics using meta-analyses. Relevant studies up to April 2018 were identified and eligible studies recorded medication use and body mass index in asthmatic adult humans. Obese asthmatics are more likely to use most classes of asthma medications, including oral corticosteroids [odds ratio (OR): 1.76; 95\% CI: 1.53, 2.02; $p<0.001$ ], long-acting $\beta$-agonists (OR: 1.66; 95\% CI: 1.23, 2.24; $p<0.001$ ), inhaled corticosteroids (OR: 1.35; 95\% CI: 1.14, 1.59; $p<0.001$ ), leukotriene receptor antagonists (OR: 1.36; 95\% CI: 1.17, 1.60; $p<0.001$ ), short-acting $\beta$-agonists (OR: 1.38; 95\% CI: 1.10, 1.73; $p=0.005$ ) and anticholinergics (OR: $1.62 ; 95 \%$ CI: 1.16, 2.24; $p=0.004$ ) compared to healthy weight asthmatics. Inhaled corticosteroid dose in asthmatics was also significantly increased by obesity (mean difference $(95 \% \mathrm{CI})$ : 166.92 (86.06-247.77) $\mu \mathrm{g} /$ day; $p<0.001)$. Our review and meta-analyses supports the hypothesis that obesity is associated with reduced response to asthma pharmacotherapies, by providing evidence that obese asthmatics have increased asthma medication use at increased doses.

\subsection{The Effects of Macronutrients on Bronchodilator Responsiveness in Asthma}

C. Thompson ${ }^{1}$, B. Berthon ${ }^{1}$, H. Scott ${ }^{1}$, P. Gibson ${ }^{1}$, P. Young ${ }^{2}$, B. Oliver ${ }^{2}$ and L. Wood ${ }^{1}$

1 Centre for Healthy Lungs, Hunter Medical Research Institute, University of Newcastle, Callaghan, NSW, Australia

${ }^{2}$ Woolcock Institute of Medical Research, Sydney, NSW, Australia

Obesity in asthma is associated with worse symptoms despite increased use of asthma medications, including bronchodilators. We aimed to examine the effect of dietary macronutrient overload on response to the bronchodilator, salbutamol, in the airways of obese and non-obese asthmatics. This was a randomised, cross-over, acute feeding study in 23 asthmatic adults $(n=12$ non-obese, $n=11$ obese). Subjects fasted overnight, and asthma medications were withheld prior to the three study visits. Subjects completed a hypertonic challenge, concluding with the administration of $200 \mathrm{mcg}$ salbutamol. Subjects then consumed one of the three isocaloric meals enriched with either saturated fatty acids (SFA), n-6 polyunsaturated fatty acids (PUFA) or simple carbohydrates (CHO). Spirometry was performed at 2, 3 and $4 \mathrm{~h}$ after the meal. Following salbutamol and each meal, forced expiratory volume in the first second $\left(\mathrm{FEV}_{1}\right)(\mathrm{L})$ improved in non-obese subjects at 2, 3 and $4 \mathrm{~h}$. However, following the high SFA meal, obese subjects demonstrated no improvement in $\mathrm{FEV}_{1}$ (L) and had an attenuated forced vital capacity (FVC) (L) over the 4-h period. In obese asthmatics only, the bronchodilator response was significantly lower following the SFA versus CHO meal $(\triangle \mathrm{FEV} 1(\mathrm{~L})$, $p=0.004)$. Therefore, a high SFA meal suppressed salbutamol recovery in obese asthmatics.

\subsection{Association between BMI and Food Liking in Thai and Australian Young Adults}

U. Wanich ${ }^{1}$, L. Riddell ${ }^{2}$, D. Sayompark ${ }^{3}$, M. Mohebbi ${ }^{4}$, S. Cicerale ${ }^{1}$, G. Liem ${ }^{1}$ and R.S.J. Keast ${ }^{1}$

${ }^{1}$ Centre for Advanced Sensory Science, Deakin university, Melbourne, VIC, Australia

2 Institute for Nutrition and Physical Activity Research, Deakin University, Melbourne, VIC, Australia

3 Faculty of Science and Technology, Rajamangala University of Technology Tawan-ok, Chonburi, Thailand

${ }^{4}$ Biostatistics Unit, Faculty of Health, Deakin University, Melbourne, VIC, Australia

An individual's liking for food maybe associated with Body Mass Index (BMI) due to its influence on food consumption. This study investigates the association between BMI and food liking in young adults from Australia and Thailand. Food liking data were collected via an online Food Liking Questionnaire (FLQ) and liking scores calculated for overall liking and liking of grains, vegetables, fruits, dairy, animal proteins, plant-based proteins, fat and oil, sweet foods, salty foods and alcohol. Relationship between liking scores and BMI (calculated from self-reported height and weight) was 
assessed using linear regression models including country and gender. Data were available from $n=4161$ participants $(\mathrm{BMI}=22.23 \pm 0.06$, age $=20.62 \pm 07$ years, female $=71.5 \%$, Thai $=52.5 \%$ ). Significant differences in food liking scores were observed between gender and country $(p<0.001)$. The only significant association with BMI was liking of animal proteins $(B=0.19[0.11,0.28], p<0.001)$, where an increased liking was associated with a higher BMI. No other associations between food liking and BMI were observed. This study demonstrates little evidence of an association between food liking and BMI but gender and cultural variation in liking was evident. These data will be used in future studies exploring the association between liking and dietary intake.

\subsection{Supermarket Food and Beverage Expenditure as a Proxy for Consumption Provides a Crucial Part of a} National Food and Nutrition Monitoring and Surveillance System

\section{P. Atyeo}

Australian Bureau of Statistics, Lyneham, ACT, Australia

A long-standing public health information goal in Australia has been to establish a national food and nutrition monitoring and surveillance system. Information addressing food behaviour and nutritional status is provided through infrequent national nutrition surveys but a reliable data source which can monitor trends in apparent consumption is a crucial yet missing part of the public health information picture. Since 2014, ABS has been collecting transactions data (TD) to measure price change for the Consumer Price Index. Each food item in the TD was coded to the AUSNUT 2011-13 database. The proportion of expenditure on selected food groups (from total food and beverage expenditure) is used as proxy for relative consumption. The ABS' 2015-16 Household Expenditure Survey was used to calibrate expenditure proportions to account for the varying propensity to purchase particular foods from supermarkets. Results show time trends such as declines in expenditure on sugar sweetened beverages (SSBs) and a socioeconomic gradient consistent with consumption data seen in the survey data. A timely and ongoing data source based on TD has the potential to inform on trends and patterns across several important positive and negative risk factor food groups such as fruit, vegetables, milk, nuts, processed meat, SSBs and confectionary.

\subsection{Usual Intake of Trans Fatty Acids by Australian Adults in 2011-12}

\section{K. Bathgate ${ }^{1}$, D. Mackerras ${ }^{1}$, T. Hambridge ${ }^{1}$, J. Boorman ${ }^{1}$ and P. Atyeo ${ }^{2}$ \\ ${ }^{1}$ Food Standards Australia New Zealand, Kingston, ACT, Australia \\ ${ }^{2}$ Australian Bureau of Statistics, Canberra, ACT, Australia}

The World Health Organization (WHO) recommends that population intake of trans fatty acids should be below $1 \%$ energy per day. As heart disease is the primary concern for this nutrient, 'per day' would refer to intake measured over the long term and not from a single day. The 2011-12 Australian National Nutrition and Physical Activity Survey data were used to describe the distribution of trans fat expressed as a \% of energy for adults aged 19 years and older, calculated three different ways. When calculated using day 1 only, the average of the two days of available data and the NCI method, respectively, 95th percentile of intake declined from 1.3 to 1.1 to $0.9 \%$ energy for both men and women. The proportion with intakes above the WHO criterion for day 1 only were $12.7 \%$ (men), $11.9 \%$ (women), and using the NCI method were $1.9 \%$ (men) and $1.1 \%$ (women). These results highlight the importance of matching the data and analysis method to the basis on which the health based guidance value was established to correctly evaluate risks associated with short or long term health effects as appropriate.

\subsection{Assessment of Affordability of Food in the Illawarra Using Two Healthy Food Basket Surveys}

K. Charlton ${ }^{1}$, P. Frean ${ }^{1}$, K. Walton ${ }^{1}$ and A. Lee ${ }^{2}$

${ }^{1}$ University of Wollongong, Wollongong, NSW, Australia

${ }^{2}$ Sax Institute, Brisbane, QLD, Australia 
The biannual Illawarra Healthy Food Basket Survey (IHFB) has assessed the affordability of a food basket since 1999 with costs between $27 \%$ and $35 \%$ of household (HH) income. For comparability across states, use of the Healthy Diets Australian Affordability Survey of Food Pricing (ASAP) is proposed. We aimed to assess the household affordability of healthy food baskets in the Illawarra, NSW, using the IHFB and ASAP survey instruments. The IHFB survey (57 items) collected food prices from supermarkets, butchers and greengrocers in five suburbs across three socioeconomic strata in May 2017. The ASAP survey (44 items), costed simultaneously, also included liquor, fast food and take-away items. Costs of baskets were compared to average household income and welfare payments for a reference family of four to ascertain affordability. Both surveys indicated that a healthy food basket was unaffordable for households reliant on welfare payments, with cost exceeding the cut-off of $30 \%$ household income. For households with average incomes, the cost of a healthy basket assessed using the IHFB and ASAP surveys represented $25 \%$ and $21 \%$ of income, respectively. Strategies to address access to adequate and nutritious food are required to ensure food security for vulnerable groups.

2.141. Vegetarian-Based Dietary Patterns and Their Relationship with Inflammatory and Immune Biomarkers: A Systematic Review and Meta-Analysis

\section{J.C. Craddock, Y.C. Probst, E.P. Neale and G.E. Peoples}

School of Medicine, Faculty of Science Medicine and Health, University of Wollongong, Wollongong, NSW, Australia

Dietary patterns with substantial proportions of energy from plant sources have been associated with favourable biomarkers of low-grade inflammation. Less is known of the relationship between 'vegetarian-based' dietary patterns and markers of inflammation and immune status. This systematic review and meta-analysis (PROSPERO, CRD42016039043; 12 May 2016) aimed to determine the relationship between vegetarian-based dietary patterns and inflammatory and immune markers. PubMed, Medline and Cochrane scientific databases were searched. Random effects meta-analyses were conducted to assess the weighted mean difference (WMD) for each outcome variable (C-reactive protein (CRP), tumour necrosis factor-alpha (TNF- $\alpha$ ), fibrinogen, natural killer cells, leukocytes, lymphocytes, thrombocytes, interleukins and immune globulins) between vegetarian and nonvegetarian study groups. Thirty observational and ten intervention studies were included in the review. Pooled effects of vegetarian-based dietary patterns were associated with significantly lower concentrations of CRP (WMD $-0.61 \mathrm{mg} / \mathrm{L}[-0.91,-0.32) p=0.001]$, fibrinogen (WMD $-0.22 \mathrm{~g} / \mathrm{L}$ $[-0.41,-0.04] p=0.02)$ and total leukocyte $\left.\left(\mathrm{WMD}-0.62 \times 10^{3} / \mu \mathrm{L}\right)[-1.13,-0.10] p=0.02\right)$ compared to those following nonvegetarian dietary patterns in observational studies. Insufficient data was identified for a meta-analysis of intervention studies. This study provides evidence for associations between vegetarian-based dietary patterns and lowered serum CRP, fibrinogen and total leukocyte concentrations.

\subsection{Electronic Tools to Support and Audit Healthy Food Environments}

M. Crino, A.-M. Healy, E. Munn, B. Tang, T. O'Connell, W. Croning, S. Chang and M. Cobcroft

NSW Ministry of Health, North Sydney, NSW, Australia

Food environments in Australia are dominated by unhealthy choices, contributing to poor diets and risk of chronic disease. In 2017, NSW Health relaunched a healthy food and drink policy that applies to food outlets in health facilities. Key limitations to implementation of the previous policy include a lack of systematic monitoring and reporting, and inadequate implementation support. To monitor and report on the achievement of a healthy food and drink offering for staff and visitors, NSW Health has developed a mobile application-tool to collect data which is linked to a food and drink database and reporting system. The tool has been designed for users with limited nutrition knowledge who can complete the audit in less than $30 \mathrm{~min}$. Implementation is additionally supported 
by an online food and drink look-up tool that allows users to easily find and supply healthier choices. These tools enable implementation and auditing of the food and drink offering in NSW Health food outlets, including cafés and vending machines, against a set of 13 food and drink practices, one of which is the removal of sugar-sweetened beverages. Adoption of the tools provides a standardised approach to annual state-wide auditing, strengthens accountability and achievement of the policy.

2.143. The FIFO Nutritionists: Teaching School Students Healthy Eating and Cooking Skills in Regional and Remote WA as Part of Foodbank WA's Food Sensations ${ }^{\circledR}$ Program

\section{F. Foulkes-Taylor and L. Thorne}

Foodbank WA, Perth Airport, WA, Australia

Foodbank WA's (FBWA) Healthy Food for All ${ }^{\circledR}$ (HFFA) team deliver the Food Sensations ${ }^{\circledR}$ (FS) program, which is free to all schools registered with the School Breakfast Program (SBP). The FS sessions include interactive activities to teach children key nutrition principles, and hands-on cooking workshops to enhance skills, knowledge and confidence in the kitchen. Since the development of the HFFA Regional Strategy in 2010, public health nutritionists from FBWA have travelled over 110,000 km to regional and remote schools across WA to deliver FS to over 8000 children-from Wyndham down to Albany and every region in between. The process of planning for a regional FS trip involves months of organisation, requiring meticulous attention to detail but allowing for flexibility and strong communication between HFFA staff, regional partners and schools. The HFFA Regional team face many challenges in executing a trip successfully, including tailoring a standard program to each region and target group, and coordinating logistical details such as flights, hire cars, bulk grocery orders and accommodation. Building capacity through training relevant stakeholders is a key strategy in enhancing the longevity of our health messages, and promoting ongoing nutrition education in schools and communities after the Regional team return to Perth.

2.144. Parent's Attitudes towards, and Demand for, the Availability of Healthy Food Options on Kids Menus at Full-Service Restaurants

K. Freeman ${ }^{1}$ and T. Lawlis ${ }^{2}$

${ }^{1}$ The Healthy Eating Hub, Harrison, ACT, Australia

${ }^{2}$ School of Rehabilitation and Exercise Sciences, University of Canberra, Canberra, ACT, Australia

Childhood obesity is a public health concern with foods consumed away from home at restaurants contributing to this epidemic. This study aimed to understand parent attitudes and demand for healthy food options on kid's restaurant menus. The study comprised two parts. Part one: development of a healthier, commercially viable kid's menu. Part two: 828 parents or carers of children aged 2-16 years from Canberra, Australia completed a quantitative online survey. Chi-square analysis was conducted to determine relationships between preferences for healthy food items and the demographic data. There was a significant reduction in the mean \pm SD energy content of main dishes from the old to the new menu $(2028.4 \pm 679.1 \mathrm{~kJ}$ vs. $1333.9 \pm 528.3 \mathrm{~kJ}, p<0.05)$. Of the total sample, 637 parents $/$ carers reported the availability of healthy food on a kids menu was important to them $(p<0.05)$. Four of the five new, healthier menu items were frequently $(47-74 \%)$ chosen by parents and all five menu items were rated as healthy by $>90 \%$ of parents / carers. Parents reported a positive attitude and demand for healthy food items on kid's menus at restaurants. Increasing the availability of healthy food items on kids' menus will contribute to improving the health of Australian children.

\subsection{The Benefits of High DHA Fish Oil in Human Nutrition}

S.G. Fard ${ }^{1}$, F.I. Wang ${ }^{2}$, A.J. Sinclair ${ }^{3}$, G. Elliott ${ }^{1}$ and G.M. Turchini ${ }^{4}$

${ }^{1}$ Research and Development Department, Nu-Mega Ingredients Pty Ltd., Brisbane, QLD, Australia

${ }^{2}$ Department of Food Science and Nutrition, Zhejiang University, Hangzhou, China 
${ }^{3}$ Department of Nutrition, Monash University, Melbourne, VIC, Australia

${ }^{4}$ School of Life and Environmental Sciences, Deakin University, Geelong, VIC, Australia

Fish oils contain high, but variable, levels of omega-3 fatty acids, including eicosapentaenoic acid (EPA) and docosahexaenoic acid (DHA). Although the two fatty acids have traditionally been considered together, different physiological effects of EPA and DHA have recently been reported. The aim is to highlight the effects of high DHA fish oils on various human health conditions. To retrieve the relevant studies to be used in this review, a search strategy using Medline/PubMed, ScienceDirect, Web of Science and EMBASE was conducted. A total of 113 studies, including human studies, follow-up studies and animal studies, were reported and analysed separately. DHA appears to play important roles, different to those of EPA, in the heart and cardiovascular system, the brain and visual function, inflammation and immune function and growth/body mass index. Important differences have been reported in the possible physiological responses to increased dietary DHA administration relative to age. Vastly different roles and effects were noticed in infants, adults, and elderly individuals. This presentation will generate a far more detailed understanding of the need of DHA in different age groups for different medical aspects which will have a great impact on the research sector as well as industry sector.

\subsection{The Shape of the Vitamin A Nutrient Intake Distribution Might Vary over Time}

A. Messina ${ }^{1}$, T. Hambridge ${ }^{2}$ and D. Mackerras ${ }^{2}$

${ }^{1}$ University of Wollongong, NSW, Australia

${ }^{2}$ Food Standards Australia New Zealand, Kingston, ACT, Australia

One challenge for estimating usual nutrient intakes in populations using a small number of days of data from respondents is the shape of the underlying distribution. In the 1995 National Nutrition Survey, vitamin A intake distributions were skewed, therefore they were log-transformed to enable longer term intake to be estimated. We explored whether the same was true for the 2011-12 National Nutrition and Physical Activity Survey. We calculated vitamin A intakes using day 1 only and the second day adjustment method using the untransformed data and after taking the natural logarithm. We compared these to the results derived by the Australian Bureau of Statistics using the National Cancer Institute (NCI) method which selects the most appropriate data transformation. The results for boys aged 14-18 years were typical of other age-sex groups. The mean (5th and 95th percentile) for day 1 only was $754(114,2166) \mu \mathrm{g}$ RE and for the NCI method $753(426,1177) \mu \mathrm{g}$ RE. Results for the untransformed second day adjusted data were $754(470,1380) \mu \mathrm{g}$ RE compared to $564(236,1067)$ $\mu \mathrm{g}$ RE after logarithmic transformation. In the 2011-12 survey, logarithmic transformation would not seem to be preferable.

\subsection{Availability and Price of Healthier Food Choices and Association with Obesity Prevalence in New Zealand Māori}

R. Jani ${ }^{1}$, E. Rush ${ }^{2}$, N. Crook ${ }^{3}$ and D. Simmons ${ }^{4}$

${ }^{1}$ University of Canberra, Bruce, ACT, Australia

2 Centre for Physical Activity and Nutrition, Auckland University of Technology, Auckland, New Zealand

${ }^{3}$ Lakes District Health Board, Rotorua, New Zealand

${ }^{4}$ Department of Medicine, Western Sydney University, Sydney, NSW, Australia

Examine availability and price of healthier foods-vs.-regular counterparts and their association with obesity. A cross-sectional survey of weight and height among Māori in two urban and 96 rural areas in the Waikato/Lakes Districts, NZ, was undertaken. Availability of 11 'healthier' foods in fast food outlets was examined by location and income. In supermarkets, five-specific 'regular' foods were scored against 'healthier' counterparts (white vs. wholemeal bread, with-skin vs. skinless chicken, 
regular vs. trim meat, standard vs. trim milk and sugar-sweetened beverages vs. water) for in-store availability and price according to the Nutrition Environment Measures Survey. Overall, 3817 Māori (BMI: women: $32.9 \pm 7.8 \mathrm{~kg} / \mathrm{m}^{2}$; men: $33.1 \pm 6.7 \mathrm{~kg} / \mathrm{m}^{2}$ ) were included with 451 food outlets in two urban clusters and 698 food outlets in 96 rural clusters. Fast foods: The availability of healthier food choices was higher for 8/11 items in rural and low-income areas than urban and high-income areas. Multivariate analysis considered location and income as cofactors. No association observed between number of fast food outlets/cluster and healthier foods/cluster with obesity prevalence (General/Māori BMI cut-offs). Supermarkets: Water was cheaper than sugar-sweetened beverages and negatively associated with obesity prevalence (General $\mathrm{r}=-0.53, p=0.03$; Māori $\mathrm{r}=-0.53, p=0.03$ ); high availability scores for trim milk compared to standard milk correlated with higher obesity prevalence (General $\mathrm{r}=0.49, p=0.04$; Māori $\mathrm{r}=0.57, p=0.01$ ). Bottled water vs. sugar-sweetened beverages prices was inversely associated with obesity. This supports the argument to regulate the availability and price of sugar-sweetened beverages in NZ.

\subsection{The Social Implications of Food Insecurity Experienced by Western Australian Single-Parent Families}

N. Philippson ${ }^{1}$, P. Chang ${ }^{1}$, A. Devine ${ }^{2}$ and T. Lawlis ${ }^{3}$

${ }^{1}$ School of Arts and Humanities, Edith Cowan University, Joondalup, WA, Australia

2 School of Medical \& Health Sciences, Edith Cowan University, Joondalup, WA, Australia

${ }^{3}$ Faculty of Health, University of Canberra, Canberra, WA, Australia

The present study explored the lived experience of food insecurity in Western Australian single-parent families. In depth-interviews were conducted with ten single-parents who had a primary school-aged child and had accessed food relief within the previous 12-month period. An interpretative phenomenological approach was used to analyse the transcripts for themes. Domestic violence, in-group and out-group behaviour and mental health issues were reported. Domestic violence led to fear of the outside world and social isolation. Accessing food relief was problematic and resulted in frustration and anger toward government agencies, family and other community members. Differences in entitlement contributed to prejudice against other users of food relief. Expectations of a perfect family and a perceived pressure of having to maintain an illusion of normality contributed to parents experiencing anxiety and depression. As a consequence, children in food-insecure households were at risk of disordered eating, increased school absence, the incapacity to attend excursions and mandatory school activities and extracurricular sports and social activities. Food insecurity is an emerging public health issue; this study gave participants an opportunity to express personal thoughts and feelings about their experience of food insecurity.

\subsection{Correlates of Sugar-Sweetened Beverage Intake at Eating Occasions in Australian Young Adults}

R.M. Leech, F.J. Pendergast, A. Worsley and S.A. McNaughton

Institute for Physical Activity and Nutrition, Deakin University, Melbourne, VIC, Australia

Sugar-sweetened beverages (SSBs) are a major source of excess caloric intake among young adults, yet little is known about the correlates of SSB intake at eating occasions (EO). This study examines the individual and situational correlates of SSB intake, using real-time assessment of young adults' EO. Dietary and sociodemographic data were collected during the Measuring Eating in Everyday Life (MEALS) study ( $n=680$ adults, $18-30$ y). Participants reported all foods and beverages consumed over 3-4 nonconsecutive days using a smartphone food diary app. For every $\mathrm{EO}$, the situational characteristics (eating and purchase location, presence of others and activities while eating) were recorded. Associations for individual and situational characteristics with SSB intake at beverage-containing EO were analysed using multilevel logistic regression. Of the 2439 beverage-containing EO reported by SSB consumers $(n=283), 483(20 \%)$ contained a SSB. Having a lower than tertiary education (odds ratio [95\% confidence interval]: $1.53[1.16,2.01]$ ) and purchasing 
beverages from a convenience outlet, compared to a supermarket/grocery store (4.57 [2.84, 7.35]), were associated with SSB intake. In conclusion, SSB intake was associated with individual and situational factors. Future research should explore how EO containing SSBs differ from other beverage $\mathrm{EO}$, in relation to their accompanying foods.

\subsection{Evaluation on The Thyroid Function And Urinary Iodine Level for Chinese Pregnant Women}

M. Li, L. Yang, X. Yang and H. Jiang

National Institute for Nutrition and Health, Chinese Center for Disease Control and Prevention, Beijing, China

We aimed to evaluate thyroid function and urinary iodine level for pregnant women at different stages of China. Plasma samples were obtained from pregnant women that participated in the 2015 Chinese Adult Chronic Disease and Nutritional Surveillance. Urinary iodine concentration (UIC) was measured by using a catalytic spectrophotometric method of arsenic and cerium. Plasma levels of thyrotrophin (TSH), free triiodothyronine (FT3), free thyroxine (FT4), anti-thyroid peroxidase antibody (TPOAb) and anti-thyroglobulin antibody (TGAb) were determined by using an automated chemiluminescence immunoassay analyser. A total of 6173 urinary samples collected from pregnant women residing in different regions of China were analysed for UIC, which median UIC was $146 \mu \mathrm{g} / \mathrm{L}$. After exclusive the positive TPOAb and TGAb, a total of 2097 plasma samples collected from pregnant women at three trimesters of China were analysed for levels of TSH, FT3 and FT4, then judged the prevalence for different types of abnormal thyroid function and normal prevalence. The rate of normal thyroid function for this cohort was more than $85 \%$. The most common type of abnormal thyroid function that was detected was subclinical hypothyroidism, and the prevalence of subclinical hypothyroidism was $6.8 \%$. Compared with 2010 nutritional surveillance, the iodine status of Chinese pregnant women is generally adequate.

2.151. Comparison of Two Nutritional Screening Tools to Detect Nutritional Risk in The Patients with AIDS and Hepatitis $C$

Q. Lu and Y. Sun

Zhengzhou University, Zhengzhou, China

Malnutrition is an important factor affecting the development of AIDS and Hepatitis C. The aim of the study was to investigate the nutritional status and compare two nutritional screening tools in patients with different diseases. A cross-sectional study with MNA (Mini Nutritional Assessment) and NRI (Nutritional Risk Index) was performed. Take the diagnostic criteria that BMI $<18.5$ or WL $\%>5 \%$ or the concentration of ALB $<35 \mathrm{~g} / \mathrm{L}$ as the gold standard. A total of 153 patients were included in the study. The prevalence of patients with nutritional risk in AIDS patients was $10.0 \%$ with MNA, $11.8 \%$ in patients with hepatitis $C$ and $20.0 \%$ in AIDS patients with hepatitis $C$ infection. According to NRI, the prevalence was $10.0 \%, 17.7 \%$ and $15.6 \%$, respectively. The results of diagnostic tests in AIDS patients were an area under the curve receiver operating characteristic of 0.674 for MNA and 0.502 for NRI; in patients with hepatitis C, 0.868 for MNA and 0.531 for NRI; and in AIDS patients with hepatitis $C$ infection, 0.654 for MNA and 0.760 for NRI. MNA might be a better screening tool than NRI for detecting the risk in patients with hepatitis $C$.

\subsection{Is A Meta-Analysis Always the Best Summary of A Body of Evidence}

D. Mackerras

Food Standards Australia New Zealand, Canberra, ACT, Australia

Meta-analysis is a weighted average and a common way of summarising data from a literature review. Two situations when this is problematic are when authors do not present results in a common 
format and when there is a nonlinear dose response. To update several previous systematic reviews and government reports, PubMed was searched in December 2016 to identify studies linking maternal or cord blood perflurooctanoic acid (PFOA) concentration and infant birthweight. An existing meta-analysis included only studies which expressed results as a linear association. Two of the eight new studies could be added and this reduced the effect size from -18.9 to $-11.9 \mathrm{~g} / \mathrm{ng} / \mathrm{mL}$ PFOA. The studies which could not be included in the meta-analysis had typically analysed data using a log linear curve and a plateau was noted in several studies. Consequently, there was an important discrepancy in results observed at higher concentrations and those extrapolated from the meta-analysis. A further set of studies only allowed the direction of the association to be assessed or simply reported 'not significant'. This highlights the need to examine all studies which meet inclusion criteria carefully, and not to discard studies that meet inclusion criteria but do not easily fit into a meta-analysis.

\subsection{Estimation of Energy Content of Larger Portions Using A Portion Size Quiz}

M.E. Blair ${ }^{1}$, M. Dean ${ }^{2}$, M. Adamski ${ }^{1}$, S.J. Gibson ${ }^{1}$, H. Truby $^{1}$, M. Reid ${ }^{3}$, M.B.E. Livingstone ${ }^{4}$ and T.A. McCaffrey ${ }^{1}$

${ }^{1}$ Monash University, Notting Hill, VIC, Australia

${ }^{2}$ Queens University, Belfast, UK

${ }^{3}$ RMIT University, Melbourne, VIC, Australia

${ }^{4}$ University of Ulster, Coleraine, UK

Food portion sizes (PS) have increased over recent decades. This study aimed to explore the association between nutrition knowledge (NK) and ability to estimate energy (kJ) content from photographs of foods. Participants were recruited via Monash University's MOOC "Food as Medicine" and snowballing. Participants completed an online Portion Size Quiz (PSQ) that tested their ability to estimate the energy content of a "large" portion of eight meals (kJ of a "standard" portion was supplied). NK was measured using objective and subjective measures including validated questions. Participants ( $n=1440,86 \%$ female) were aged $25-34$ years $(25 \%)$, born in Australia (37\%) and had completed a Bachelor degree or higher (30\%). Objective (median score $90 \%$ ) and subjective (median score $80 \%$ ) NK were high, but performance on the PSQ (median score 50\%) was low. There was no correlation between objective NK and PSQ scores $(r=0.039, p=0.135)$. Subjective NK was weakly correlated with PSQ scores $(r=0.069, p=0.009)$. High objective NK did not result in an increased ability to estimate energy content of portions. Strategies to reduce energy intake may be better directed to address portion size norms in food service settings rather than try to improve consumer knowledge about portions and their energy content.

\subsection{Assessment of Dietary Sodium Intake Using Dietary Assessment versus 24-h Urine: A Systematic Literature Review}

R.M. McLean ${ }^{1}$, V.L. Farmer ${ }^{1}$, A. Nettleton ${ }^{1}$, C.M. Cameron ${ }^{1}$, N.R. Cook $^{2}$ and N.R.C. Campbell ${ }^{3}$

${ }^{1}$ University of Otago, Dunedin, OTAGO, New Zealand

2 Department of Medicine, Brigham and Women's Hospital, Boston, MA, USA

${ }^{3}$ University of Calgary, Calgary, AB, Canada

Although dietary assessment methods are often used in epidemiological studies, twenty-four hour $(24 \mathrm{~h})$ urine is generally considered to be a more accurate measure of dietary sodium intake. This systematic literature review investigates whether $24 \mathrm{~h}$ diet recall, diet records and Food Frequency Questionnaires (FFQs) are reliable and valid ways to measure usual dietary sodium intake. We included studies which compared dietary assessment methods and $24 \mathrm{~h}$ urinary collection for assessment of sodium intake in the same participants. The results of 20 studies using $24 \mathrm{~h}$ diet recall, 10 studies using diet records and 18 studies using FFQs were reviewed. Design and statistical analysis varied widely between studies. Reported correlations between estimates were generally low and ranged from 0.16 
to 0.72 for $24 \mathrm{~h}$ diet recall, 0.11 to 0.49 for diet records and -0.10 to 0.36 for FFQs. Only three studies reported Bland-Altman analysis, with all showing poor agreement between dietary and $24 \mathrm{~h}$ urinary excretion measures. Validation studies of dietary assessment methods show generally poor agreement with $24 \mathrm{~h}$ urinary excretion for measurement of dietary sodium intake. Robust reporting of validation studies is important in order to understand the limitations of specific dietary assessment methods.

\title{
2.155. Contribution of Food Groups to Annual Sodium Purchases among Members of Discovery Vitality
} Health Programme

\author{
R. Ndanuko ${ }^{1}$, S. Peters ${ }^{2}$, J. Wu ${ }^{1}$ and B. Neal ${ }^{1}$ \\ ${ }^{1}$ The George Institute for Global Health, Sydney, NSW, Australia \\ 2 The George Institute for Global Health, Oxford, UK
}

Excess dietary sodium intake is associated with increased risk of cardiovascular diseases. The South African Discovery Vitality health programme rewards its members for purchasing healthy food products. The aim of this study was to assess the contribution of different food groups to sodium purchases among Discovery Vitality members, and estimate potential reduction in sodium upon implementation of recently introduced sodium reduction legislation (implementation due by June 2019). Purchases data collected between 2016 and 2017 from two large supermarket chains (Pick `N Pay and Woolworths supermarkets) was utilised. Foods were categorised into 16 groups in accordance with the Global Food Monitoring Group categorisation system. 7440 products with complete purchases and nutrient information were available for analysis. A total of 428,184 kg of salt was purchased annually. The highest food group contributors to total salt purchases were bread and bakery products; dairy; sauces, dressings, spreads and dips; and meat and meat products contributing $21 \%, 15.4 \%, 15.4 \%$ and $15.2 \%$, respectively. Compared to current levels, a further reduction in sodium levels of $9.3 \%$ is needed to achieve full adherence to the mandatory targets. Additional strategies will likely be required in order to achieve the $30 \%$ population salt reduction recommended by the World Health Organization.

\subsection{Understanding the Nutritional Value of Anthocyanins}

M. Netzel ${ }^{1}$, O. Wright ${ }^{2}$ and G. Netzel ${ }^{1}$

${ }^{1}$ University of Queensland, Coopers Plains, QLD, Australia

2 School of Human Movement \& Nutrition Sciences, University of Queensland, St. Lucia, QLD, Australia

There is emerging evidence from epidemiological and experimental studies that suggests that a higher consumption of anthocyanin-rich foods is associated with a reduced risk for heart disease and diabetes. To better understand the potential mode of action of these polyphenolic plant pigments, their bioavailability and metabolic fate needs to be studied in more detail. Healthy human subjects (4-12 in three different studies) received strawberry puree (147 $\mathrm{mg}$ anthocyanins / mainly as pelargonidin-3-glucosid), red grape pomace (700 mg anthocyanins/mainly as malvidin-3-glucosid), Queen Garnet plum juice (426 mg anthocyanins / mainly as cyanidin-3-glucosid) or an anthocyanin-free control in a randomised cross-over design. 24-h urine samples were collected and analysed for anthocyanins and metabolites by LC-MS. Methylated, glucuronidated and sulphated anthocyanins could be identified as the primary metabolites in all studies (total excretion between 0.18 and $1.1 \%$ of the ingested dose). Furthermore, urinary hippuric acid (microbial metabolite) was significantly $(p<0.05)$ increased after test food consumption (1.8-4.5-fold vs. control). Results from these studies suggest that metabolites, and not the 'native' fruit anthocyanins, are most likely the bioactive compounds in vivo. However, more human studies are warranted focusing on the metabolites/degradation products generated by the gut microbiota. 
2.157. Changes in Dietary Fibre Intake and Sources across Adolescence with Associated Familial Factors

O.G. Swann ${ }^{1}$, M. Kilpatrick ${ }^{1}$, M. Breslin ${ }^{1}$, T. O'Sullivan ${ }^{2}$ and W.H. Oddy ${ }^{1}$

${ }^{1}$ Menzies Institute for Medical Research, University of Tasmania, Hobart, TAS, Australia

2 Edith Cowan University, Perth, WA, Australia

Dietary fibre is essential for a healthy diet; however, data on intakes, sources and associated familial factors in Australian adolescents are limited. Dietary fibre intake determined by food frequency questionnaire was examined in adolescents from the Western Australian Pregnancy Cohort (Raine) Study 14 year $(n=1626$ (835 male)) and 17 year $(n=836$ (405 male)) follow-ups. Differences in dietary fibre intakes with age and sex were calculated using chi-squared tests and $t$-tests. Correlations of familial factors with dietary fibre intake were determined using mixed model analysis. Intake of dietary fibre decreased from $24.29 \mathrm{~g} /$ day at 14 years to $22.96 \mathrm{~g} /$ day at 17 years $(p<0.001)$, as did the quantity of dietary fibre consumed per MJ (2.60 g/MJ to $2.52 \mathrm{~g} / \mathrm{MJ}, p=0.006)$. Dietary fibre intake was lower in females than in males at 14 and 17 years $(p<0.001$ for both). Fibre intake per MJ was higher in females than males at both time points ( $p<0.001$ for both). In multivariable analysis, male sex, poor family functioning and non-Caucasian race were associated with low dietary fibre intake. Higher parental education and energy intake were associated with greater dietary fibre intake. These findings will guide further research into dietary fibre and identify those who would most benefit from public health interventions.

2.158. Comparison of Mean Salt Intake from Recent Surveys (2011 to 2017) with The Global Burden of Disease (GBD) Study 2010 Estimates

S.R. Thout ${ }^{1}$, J.A. Santos ${ }^{2}$, B. McKenzie ${ }^{2}$, K. Trieu ${ }^{2}$, R. McLean ${ }^{3}$ and J. Webster ${ }^{2}$

${ }^{1}$ The George Institute for Global Health, India, Hyderabad, Telangana, India

2 The George Institute for Global Health, University of New South Wales, Newtown, NSW, Australia

${ }^{3}$ Department of Preventive and Social Medicine, University of Otago, Dunedin, New Zealand

In 2013, the World Health Organization recommended all countries to reduce population salt intake by $30 \%$ by 2025. In the same year, a Global Burden of Disease (GBD) study established 2010 national salt intake estimates for 187 countries based on Bayesian modelling. This study aimed to systematically review studies that measured nationally-representative salt intake using 24-h urine collection since the GBD study. Electronic databases were searched from 2011 to 2017. Two reviewers assessed study eligibility based on prespecified criteria. Measured salt intake was extracted and compared with the GBD estimates. Ten studies were included, capturing data on 11,404 adult participants. Four studies were from Europe, and one each from the United States, Canada, Benin, India, Samoa and New Zealand. Mean daily salt intake ranged from 7.09 in Samoa to $10.66 \mathrm{~g} /$ day in Portugal. Measured mean salt intake from recent surveys in Italy, England and Canada were lower compared to the 2010 GBD estimates, and higher in Samoa and Benin. The study revealed that only 10 countries have published nationally-representative salt intake data using 24-h urine since 2010 and in all, salt levels remain high. Additional global efforts to lower salt intake and monitor salt strategies are urgently needed.

\subsection{An Evaluation Framework for a Healthy Food and Drink Policy for Staff and Visitors in NSW} Health Facilities

H. Trevena ${ }^{1,2}$, M. Thomas ${ }^{3}$, T. O'connell ${ }^{1}$, E. Quinert ${ }^{1}$, L. Munn ${ }^{1}$, K. Crawley ${ }^{1}$, M. Crino ${ }^{1}$, A.-M. Mackintosh ${ }^{1}$, L. Cranney ${ }^{3}$ and M. Cobcroft ${ }^{1}$

${ }^{1}$ Centre for Population Health, NSW Ministry of Health, Sydney, NSW, Australia

2 Menzies Centre for Health Policy, School of Public Health, University of Sydney, Sydney, NSW, Australia 
3 Prevention Research Collaboration, School of Public Health, University of Sydney, Sydney, NSW, Australia

A healthy food and drink policy for staff and visitors in New South Wales (NSW) health facilities ('policy') was launched in June 2017 by NSW Health. It provides best-practice guidelines for NSW Health facilities to achieve healthy food and drink in its food outlets. Evaluating its effectiveness is required to continuously improve implementation and to expand healthy food and drink approaches to other settings. This case study describes an evaluation framework to evaluate both the process of the implementation of the policy and impact, and it also includes context and case-studies in readiness for assessing scaling up. Guided by a logic model, this is a complicated 2-year evaluation (August 2017-August 2019) that requires varied stakeholders in the delivery of 12 evaluation components. Study designs and methods include a policy analysis underpinned by political science, qualitative studies using a realist approach, consumer intercept surveys and observation of food purchases, reliability and validity of an electronic tool to audit change in food and drink availability and audit results. Knowing what works, why, when and for whom will facilitate NSW Health to be a role model to support adoption of similar policy interventions in other settings that aim to promote the healthy choice as an easy choice.

(C) 2019 by the authors. Licensee MDPI, Basel, Switzerland. This article is an open access article distributed under the terms and conditions of the Creative Commons Attribution (CC BY) license (http:/ / creativecommons.org/licenses/by/4.0/). 